*wals View/Frint Document Cover Sheet

This document was retrieved from the Boeing ISEARCH System.

Accession \#: D196071708

Document \#: SD-WM-DP-104

Title/Desc:

FINAL REPORT FOR TANK 241BY103 AUGER SAMPLES 95-AUG-012 \& 95-AUG-013 
ENGINEERING CHANGE NOTICE

\begin{tabular}{|c|c|c|c|c|c|}
\hline \multirow{3}{*}{$\begin{array}{l}\text { 2. ECN Category } \\
\text { (mark one) } \\
\text { Supplenental } \\
\text { Direct Revision } \\
\text { Change ECH } \\
\text { Temporary } \\
\text { Standby } \\
\text { Supersedure } \\
\text { Cancel/Void }\end{array}$} & \multicolumn{2}{|c|}{$\begin{array}{l}\text { 3. Originator's Nane, Organization, MSIN, } \\
\text { and Telephone No. } \\
\text { Steve E. Kelly, } \\
\text { Characterization Plans, } \\
\text { Coordinajton and Reports, R2- } \\
12,376-1566\end{array}$} & \multicolumn{2}{|c|}{$\begin{array}{l}\text { 3a. USa Required? } \\
{[] \text { Yes }[X] \text { No }}\end{array}$} & $10 / 03 / 95$ \\
\hline & \multicolumn{2}{|c|}{$\begin{array}{l}\text { 5. Project Title/No./Work Order No. } \\
\text { Tank 241-BY-103 }\end{array}$} & \multicolumn{2}{|c|}{$\begin{array}{l}\text { 6. Bldg./Sys./Fac. No. } \\
\text { N/A }\end{array}$} & 7. Approval Designator \\
\hline & \multicolumn{2}{|c|}{$\begin{array}{l}\text { 8. Document Numbers Changed by this ECK } \\
\text { (includes sheet no. and rev.) } \\
\text { WHC-SD-WM-DP-104, ReV. } 0\end{array}$} & \multicolumn{2}{|c|}{$\begin{array}{l}\text { 9. Related ECN No(s). } \\
\qquad N / A\end{array}$} & $\begin{array}{l}\text { 10. Related PO No. } \\
\qquad N / A\end{array}$ \\
\hline \multirow{2}{*}{$\begin{array}{l}\text { 11a. Modification Work } \\
{[] \text { Yes (fill out Blk. }} \\
11 \mathrm{~b}) \\
{[X] \text { No (NA Blks. 11b, }} \\
11 \mathrm{c}, 11 \mathrm{~d})\end{array}$} & \multirow[t]{2}{*}{$\begin{array}{l}\text { 11b. Work Package } \\
\text { No. } \\
\text { N/A }\end{array}$} & \multicolumn{2}{|c|}{$\begin{array}{l}\text { 11c. Modification Work Complete } \\
\text { N/A }\end{array}$} & \multicolumn{2}{|c|}{$\begin{array}{l}\text { 11d. Restored to Original Condi- } \\
\text { tion (Temp. or Standby ECN only) } \\
\text { N/A }\end{array}$} \\
\hline & & \multicolumn{2}{|c|}{ Cog. Engineer Signature \& Date } & \multicolumn{2}{|c|}{ Cog. Engineer Signature \& Date } \\
\hline
\end{tabular}

12. Description of Change

This ECN is being generated in order to update/add additional information to the existing document.

13a. Justification (mark one)

Criteria Change [] Design Improvenent

As-found []

Facilitate Const

[] Environmental

[] Const. Error/Omission

[]

Facility Deactivation Design Error/Omission

13b. Justification Details

This ECN will assist in the completion of the documentation process for this document.

14. Distribution (include nane, MSIN, and no. of copies)

See attached distribution sheet.
RELEASE SIAMP

OFFICIAL RELEASE BY WHC

DATE OCT 101995

\footnotetext{
A-7900-013-2 (11/94) GEFOS5
} 


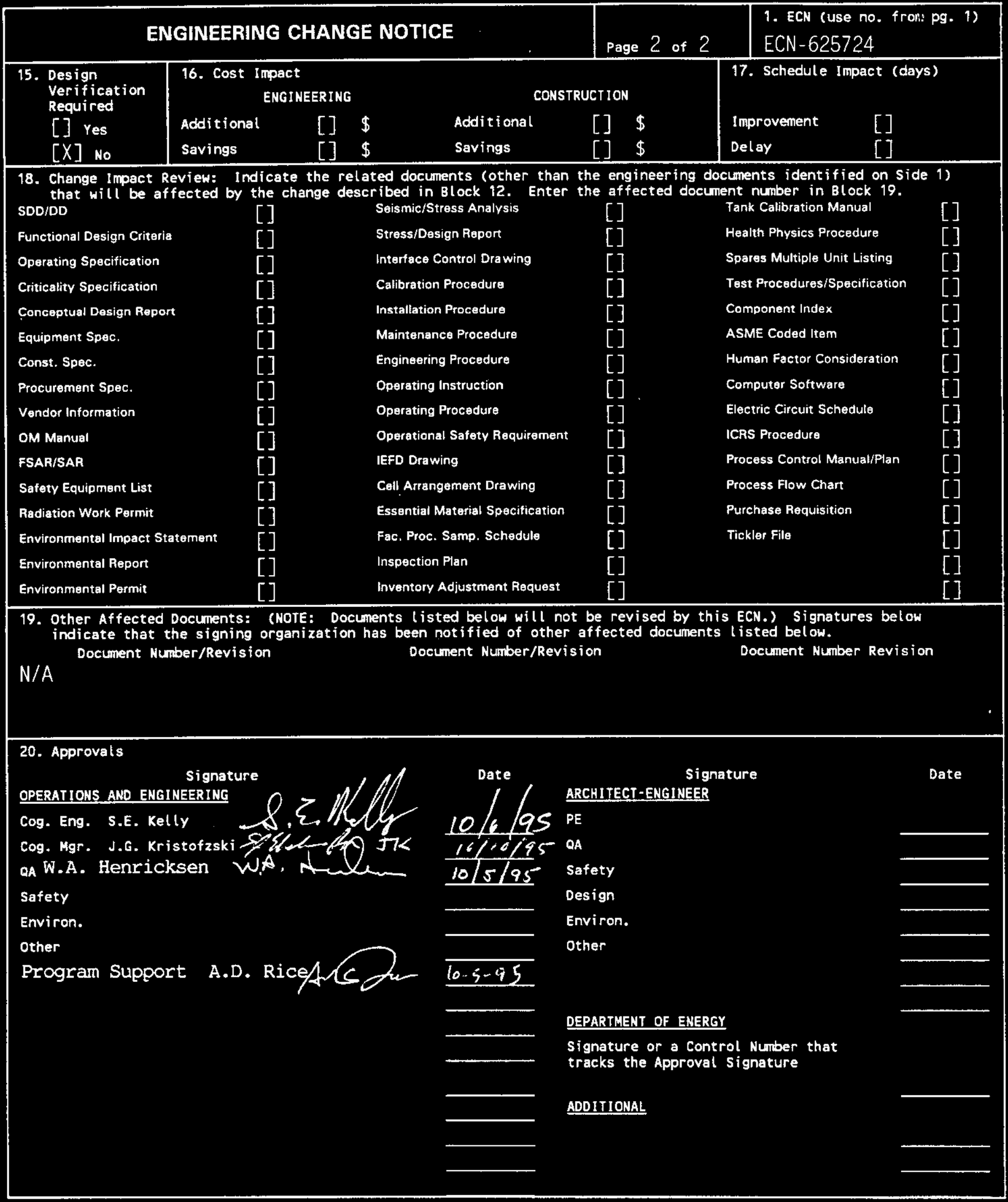




\section{RELEASE AUTHORIZATION}

Document Number: WHC-SD-WM-DP-104, REV 1

Document Title: Final Report for Tank 241-BY-103, Auger Samples 95AUG-012 and 95-AUG-013

Release Date: $\quad 10 / 10 / 95$

This document was reviewed following the procedures described in WHC-CM-3-4 and is:

APPROVED FOR PUBLIC RELEASE

WHC Information Release Administration Specialist:
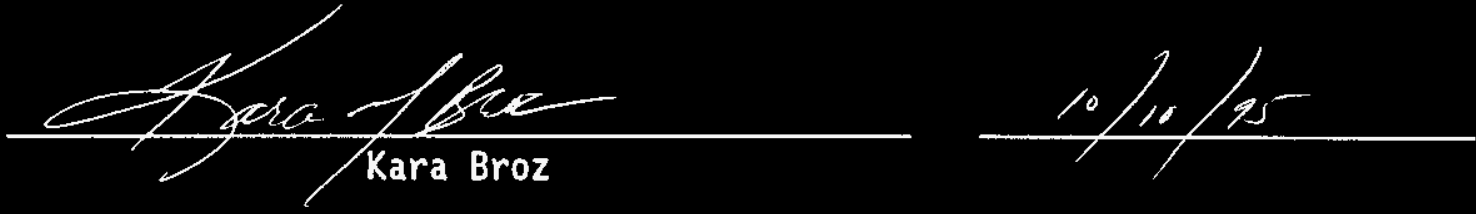

TRADEMARK DISCLAIMER. Reference herein to any specific commercial product, process, or service by trade name, trademark, manufacturer, or otherwise, does not necessarily constitute or imply its endorsement, recommendation, or favoring by the United States Government or any agency thereof or its contractors or subcontractors.

This report has been reproduced from the best available copy. Available in paper copy. Printed in the United States of America. To obtain copies of this report, contact:

West inghouse Hanford Company - Document Control Services

P.0. Box 1970, Mailstop H6-08, Richland, WA 99352

Telephone: (509) 372-2420; Fax: (509) 376-4989 
THATS PAGE INTENTIONALLY IETT BLANK 


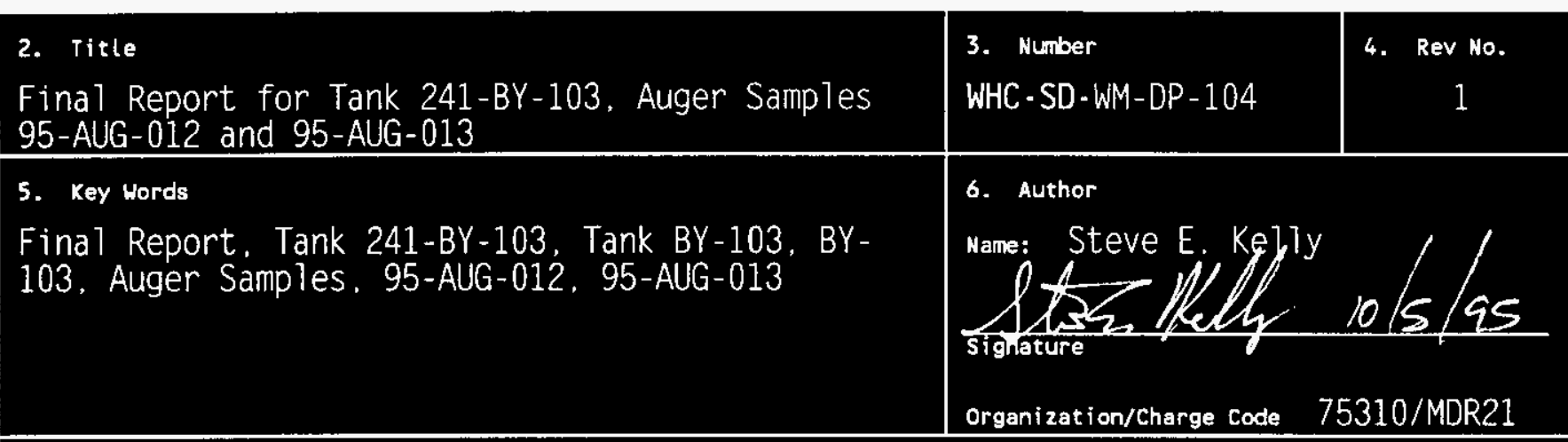

7. Abstract

N/A

8. RELEASE StAMP

OFFICIAL RELEASE
BYWHC
DATE DCT 101995
Sta 4


THIS PAGE INTENTIONALLY LEFT BLANK 


\section{RECORD OF REVISION}

$$
\begin{aligned}
& \text { (1) Docunient Number } \\
& \text { WHC-SD-WM-DP-104 }
\end{aligned}
$$

(2) Title

Final Report for Tank 241-BY-103. Auger Samples 95-AUG-012 and 95-AUG-013

\begin{tabular}{|c|c|c|l|l|}
\hline \multirow{2}{*}{ (3) Revision } & \multirow{2}{*}{ (4) Description of Change - Replace, Add, and Delete Pages } & \multicolumn{2}{|c|}{ Authorized for Release } \\
\cline { 4 - 5 } & (7) $\begin{array}{l}\text { Initially released 04/21/95 on EDT - } \\
610419 .\end{array}$ & $\begin{array}{l}\text { R.D. } \\
\text { Schreiber }\end{array}$ & J.G. KristofzSki \\
\hline & & & & \\
\hline 1 RS & Incorporate per ECN-625724. & S.E. Kelly & J.G. Kristofzski \\
\hline
\end{tabular}




\section{THIS PAGE INTENTIONALLY}

IEFT BLANK 


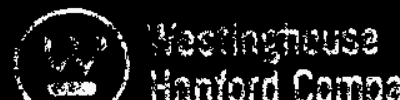

(ces) inomord Company

P.O. Box 1970 Richland, WA 99352

PART I

WHC-SD-WM-DP-104, REV. 1

ANALYTICAL SERVICES

FINAL REPORT FOR TANK 241-BY-103, AUGER SAMPLES, 95-AUG-012 AND 95-AUG-013

DATE PRINTED:

SEPTEMBER 29, 1995 
THIS PAGE INTENTIONALLY LEFT BLANK 
WHC-SD-WM-DP-104, REV. 1

TABLE OF CONTENTS

PART I FINAL REPORT FOR TANK 241-BY-103, AUGER SAMPLES 95-AUG-012 AND 95AUG-013

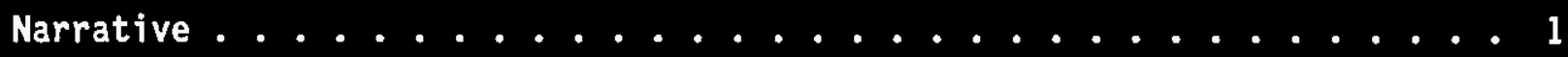

Sample Data Summary ................. 5

Chain of Custody forms ................. 8

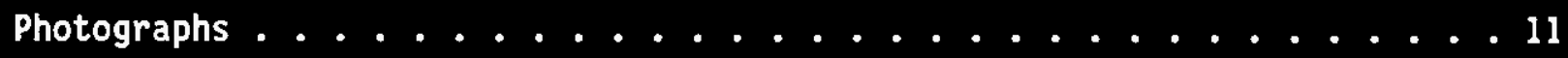

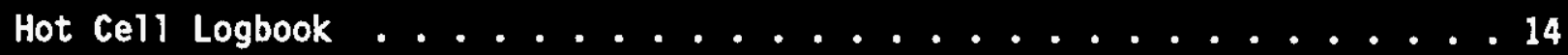

Sample Handling . . . . . . . . . . . . . . 28

Extrusion Worklist \#719 ................. 29

Extrusion Worklist \#720 ................ 30

Extrusion Workl ist \#1070.............. 31

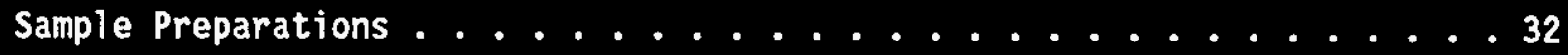

Fusion Worklist \#996................. 33

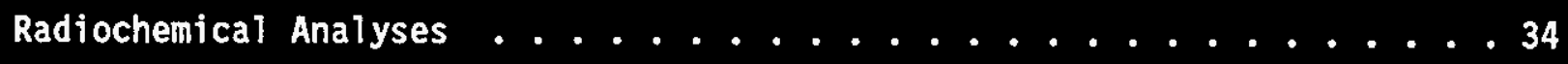

Alpha Total (AT)

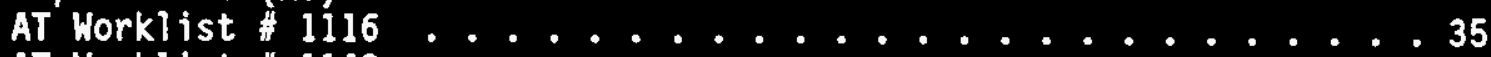

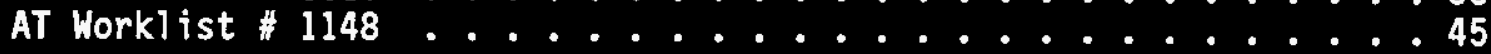

PART II 45-Day Safety Screen Results for Tank 241-By-103, Auger Samples

95-AUG-012 and 95-AUG-013 ...............55

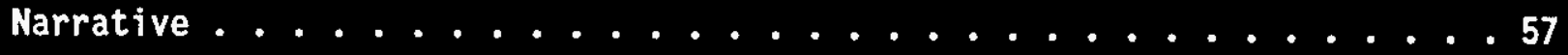

Sample Data Summary . . . . . . . . . . . . . 661

Undigested Sample Results - Direct . . . . . . . . . . . 64

Differential Scanning Calorimetry (DSC)

Worklist \#938.................... 65

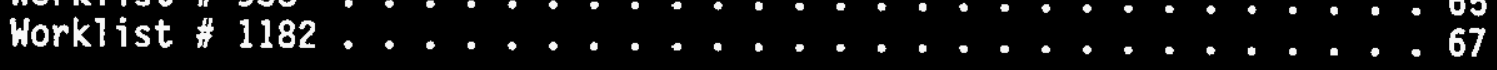

Thermogravimetric Analysis (TGA)

Workl ist \#945 .................... . 76

This Document consists of pages 1 through 84. 
THIS PAGE INTENTIONALLY LEFT BLANK 
WHC-SD-WM-DP-104, REV . 1

NARRATIVE 


\section{THIS PAGE INTENTIO
LETT BLANK \\ AIIY}




\section{WHC-SD-WM-DP-104, REV. 1 \\ FINAL REPORT FOR TANK 241-BY-103, AUGER SAMPLES 95-AUG-012 AND 95-AUG-013}

\section{ANALYTICAL SUMMARY}

Two auger samples from tank 241-BY-103 (BY-103) were received by the 222-S Laboratories and underwent safety screening analysis, consisting of

differential scanning calorimetry (DSC), thermogravimetric analys is (TGA), and total alpha activity. The TGA analys is is performed to verify a moisture content of tank waste of greater than 17 percent. Thermogravimetric analysis results for both samples were less than the limit of 17 percent moisture, and notification was made on April 20, 1995. Although the sample results were below this limit, no secondary analyses were required or performed.

\section{$\underline{\text { SCOPE }}$}

This document serves as the final report deliverable for the tank BY-103 auger samples collected on March 8, 1995 and March 10, 1995 (95-AUG-012 and 95-AUG013). Since no additional analyses have been performed, this report is identical to the 45-day report, except for the addition of the chain-ofcustody records, hot-cell work plans, subsampling worksheets, and sample extrusion photos. Each sample was received, extruded, and analyzed by the 222-S Laboratories in accordance with the Tank Characterization Plan (TCP) referenced below. Included in this report are the primary safety screening results obtained from the analyses and copies of all DSC, TGA raw data scans and total alpha results as requested per the TCP.

SAMPLE RECEIPT, EXTRUSION, AND SUBSAMPLING

Auger 95-AUG-012

Auger sample 95-AUG-012 was collected from riser 10B of tank BY-103 using a 10 inch auger bit. It was received into the 222-S Laboratories on March 10, 1995 at 1600 hours and extruded on March 23, 1995. There was no drainable liquid, but 35.85 grams of solids were collected. The solids were brownish-grey in color, looked dry and crumbly, and fell off the auger onto the sample tray after the sleeve was removed. Due to the small amount of sample obtained, all material was subsampled into one jar ( $\# 6570$ ) and was not divided into half-segments as directed in the TCP. All analysis and sample archiving requirements per the TCP were performed on this sample.

\section{Auger 95-AUG-013}

Auger sample 95-AUG-013 was collected from riser $12 \mathrm{~A}$ of tank BY-103 using a 10 inch auger bit. It was received into the 222-S Laboratories on March 10, 1995 at 1600 hours and extruded on March 24, 1995. There was no drainable liquid, but 38.09 grams of solids were collected. The solids were yellow-brown in color, looked dry and crumbly, and fell off the auger onto the sample tray after the sleeve was removed. As with the sample material from auger 


$$
\text { WHC-SD-WM-DP-104, REV. } 1
$$

95-AUG-012, the material from 95-AUG-013 was subsampled into one jar (\#6578) from which all analyses and sample archives were performed.

SAMPLE IDENTIFICATION INFORMATION

Table 1: Sample Identification

\begin{tabular}{||c|c|c|c||}
\hline $\begin{array}{c}\text { LABC0RE } \\
\text { Sample ID }\end{array}$ & Customer Sample ID & $\begin{array}{c}\text { Hot Cel1 } \\
\text { Vial \# }\end{array}$ & Analyses \\
\hline \hline S95T000320 & $95-A U G-012$, Riser 10B, Auger sample & NA & Extrusion \\
\hline S95T000507 & $95-A U G-012$, Riser 10B, Subsample & 6668 & DSC/TGA \\
\hline S95T000508 & $95-A U G-012$, Riser 10B, Fusion Digest & 6668 & Total Alpha \\
\hline S95T000509 & $95-A U G-012$, Riser 10B, Archive Sample & 6756 & Archive \\
\hline S95T000321 & $95-A U G-013$, Riser 12A, Auger sample & NA & Extrusion \\
\hline S95T000511 & $95-A U G-013$, Riser 12A, Subsample & 6810 & DSC/TGA \\
\hline S95T000512 & $95-A U G-013$, Riser 12A, Fusion Digest & 6810 & Total Alpha \\
\hline S95T000513 & $95-A U G-013$, Riser 12A, Archive Sample & 6877 & Archive \\
\hline
\end{tabular}

NA $=$ Not Applicable

\section{ANALYTICAL RESULTS}

Analytical results are presented in Tables 2, 3, 4, and 5 (pages 6 and 7) with the applicable notification limits shaded.

DSC (Energetics Content)

DSC analyses were performed under a nitrogen atmosphere using procedure LA-514-113, Rev. B-1. Using the average percent water by TGA for each sample, dry weight basis results were calculated from the wet weight bas is results. Both the wet and dry results are given in Tables 2 and 3 . For both sample S95T000507 and sample S95T000511, exotherms ranging from 5.9 to $137.6 \mathrm{~J} / \mathrm{g}$ (dry weight basis) were detected beginning at approximately $400{ }^{\circ} \mathrm{C}$; however, the magnitude of these exotherms did not approach the notification limit of $481 \mathrm{~J} / \mathrm{g}$. Due to the inhomogeneity of the samples, neither sample met the TCP specified precision criterion of $10 \%$ relative percent difference (RPD), therefore an additional analysis (triplicate) was performed for each of the two samples. Sample standard deviation values were 27.0 and 4.0 for samples S95T000507 and S95T000511, respectively. The LMCS control standards exhibited a recovery of 104.7 percent for the sample and duplicate and 99.12 for the triplicate, which are within the program's specified accuracy control limits of 90 to 110 percent recovery. 
WHC-SD-WM-DP-104, REV. 1

\section{TGA (Moisture Content)}

Weight percent water was performed under a nitrogen atmosphere using procedure LA-560-112, Rev. A-2. Results for samples S95T000507 and S95T000511 and their duplicates ranged in value from 11.63 to 33.59 percent water by weight. The marked difference in the results is most likely attributable to the inhomogeneity of the sample material. The results for both samples were less than the notification 1 imit of 17 weight percent water, although the results of the duplicate samples were greater than this limit (notification is made if the sample is analyzed at less than 17 percent water). The RPD values between the samples and their duplicates did not meet the TCP specified precision requirement of $10 \%$, therefore an additional analys is (triplicate) was performed for each of the two samples. Sample standard deviation values were 10.2 and 3.9 for samples S95T000507 and S95T000511, respectively. The Laboratory Measurement Control System (LMCS) control standard exhibited a recovery of 99.98 percent, which was within the program's specified accuracy control limits of 90 to 110 percent.

\section{Total Alpha Activity}

Although not required by the TCP for saltcake samples, analyses for total alpha activity were performed on samples S95T000508 and 595T000512. Samples were prepared by fusion digestion using procedure LA-549-141, Rev, C-2, and analyses were performed using procedure LA-508-101, Rev. D-2. A sample duplicate and spike were performed on both samples. Neither sample met the TCP specified precision criterion of 10\% RPD. Likewise, lower than normal spike recoveries caused both samples to exceed the accuracy control limits of 90 to 110 percent recovery, with values of $67.0 \%$ and $76.0 \%$. This poor spike recovery was most likely due to solids observed in the sample mount. An additional run was performed for both samples from which similar results were found. The results of the rerun are given in Tables 2 and 3 . Sample and duplicate alpha activities ranged from less than $3.17 \mathrm{E}-03$ to $2.68 \mathrm{E}-02 \mu \mathrm{Ci} / \mathrm{g}$, which were more than 1500 fold lower than the notification limit of $41 \mu \mathrm{Ci} / \mathrm{g}$.

Project Coordinator for Rev. 0 was R. D. Schreiber. Project Coordinator for Rev. I was S. E. Kelly.

REFERENCE Schreiber, R. D., 1995, WHC-SD-WM-TP-231, Revision 1, "Tank 241-BY-103 Tank Characterization Plan, dated February 27, 1995. 
THIS PAGE INTENTONAWI

LEFT BLANK 
WHC-SD-WM-DP-104, REV. 1

SAMPLE DATA SUMMARY 


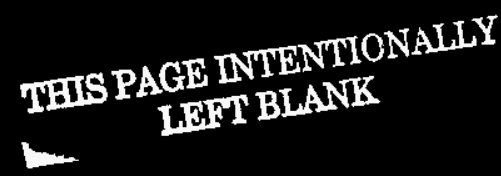


RISER: $10 \mathrm{~B}, 12 \mathrm{~A}$

SECNENT \#: 95-AUG-012

SEGMENT PORTION: $W$ thole Segment

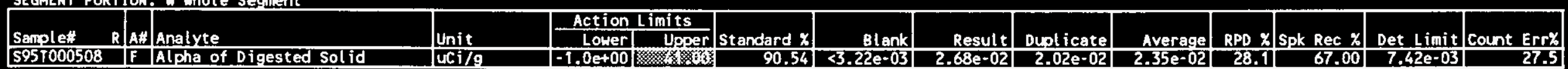

\section{$\Rightarrow$ Limit violated}

$\Rightarrow$ Selected Limit

\begin{tabular}{|c|c|c|c|c|c|c|c|c|c|c|}
\hline \multicolumn{11}{|c|}{$\begin{array}{l}\text { TANK: BY-103 } \\
\text { SEGMENT *: } 95-A U G-012 \\
\text { RISER: 10B, 12A } \\
\text { SEGMENT PORTION: Whole Segment }\end{array}$} \\
\hline \multirow[b]{2}{*}{ SAMPLE * } & \multirow[b]{2}{*}{ ANALYTE } & \multirow[b]{2}{*}{ UNIT } & \multicolumn{2}{|c|}{ ACTION LIMITS } & \multirow[b]{2}{*}{ STANDARD \% } & \multirow[b]{2}{*}{ RESULT } & \multirow[b]{2}{*}{ DUPLICATE } & \multirow[b]{2}{*}{ TRIPUCATE } & \multirow[b]{2}{*}{ AVERAGE } & \multirow[b]{2}{*}{ STDS } \\
\hline & & & LOWER & UPPER & & & & & & \\
\hline S95T000507 & DSC Exotherm using Mettler & Joules/g & \begin{tabular}{|l|} 
None \\
\end{tabular} & None & \begin{tabular}{|r|}
104.7 \\
\end{tabular} & 75.4 & 91.4 & 77.1 & 81.3 & \begin{tabular}{|r|}
8.8 \\
\end{tabular} \\
\hline $595 T 000507$ & DSC Exotherm Dry Calculated & Joules/g Dry & -1.00 & rats & N/A & 87.7 & 137.6 & 94.9 & 106.7 & 27.0 \\
\hline S95T000507 & $\%$ Water by TGA using Mottler & Percent & 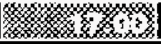 & 105.00 & 99.98 & 16800 & 33.59 & 18.76 & 22.1 & 10.2 \\
\hline (: & $\Rightarrow>$ Limit Violated & & & & & & & & & \\
\hline (1) & $\Rightarrow>$ Selocted Limit & & & & & & & & & \\
\hline
\end{tabular}




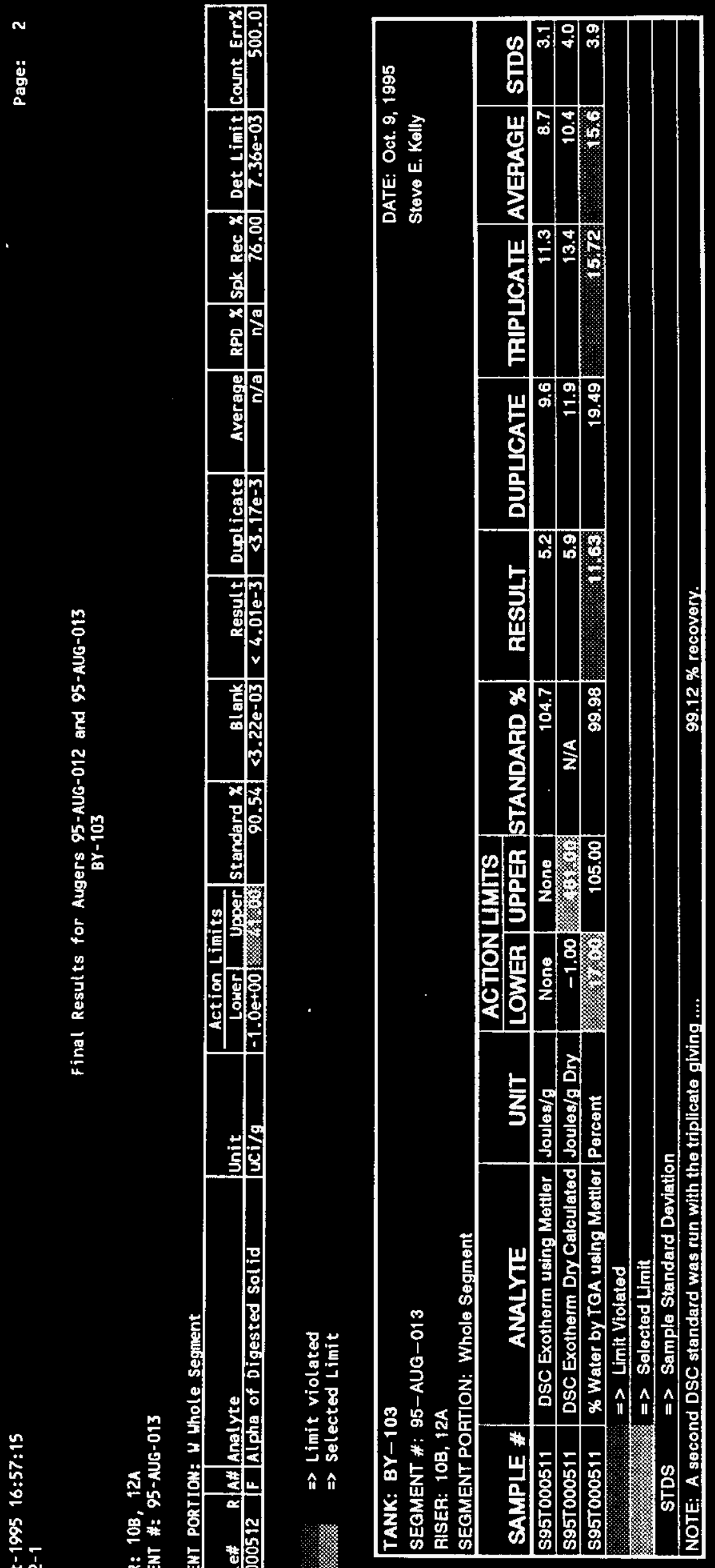


WHC-SD-WM-DP-104, REV. 1

CHAIN OF CUSTODY FORMS 
THIS PAGE INTENTIONALLY

LEFT BLANK 
CHAIN-OF-CUSTODY RECORD FOR AUGER SAMPLING

(1) Shipmont Number

(2) somplo Numbor $95-41 / 6 a-012$

Preaznit

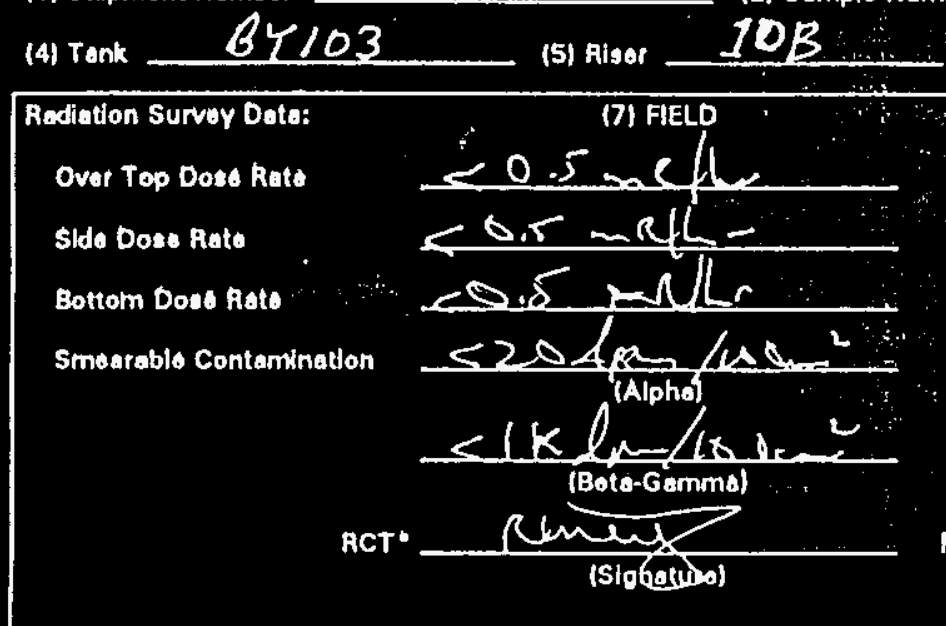

(6) Cosk sorial Numbot $C / O 22$

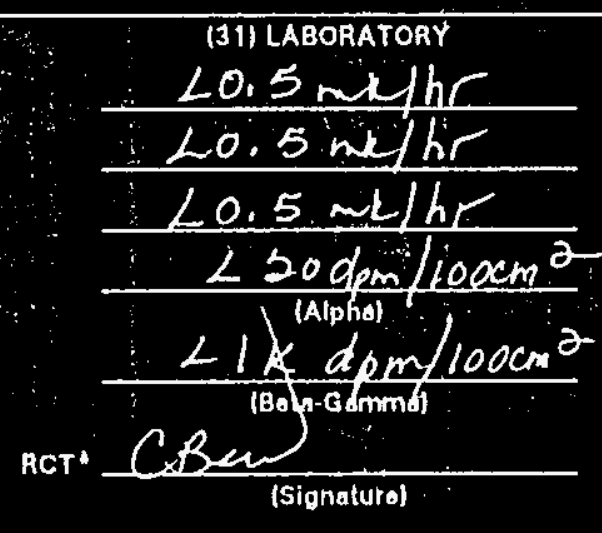
(8) Shipmont Doscription
A. Work Packago Numbor
B. Cask Soal Numbor
C. Data and Timb Samplo
Ridinovod from Tank
D. Expoctod Liquid Contont
E. Expoctód Solid Contont
F. Bose Rato Through brill String
G. Expoctod Samplo Length

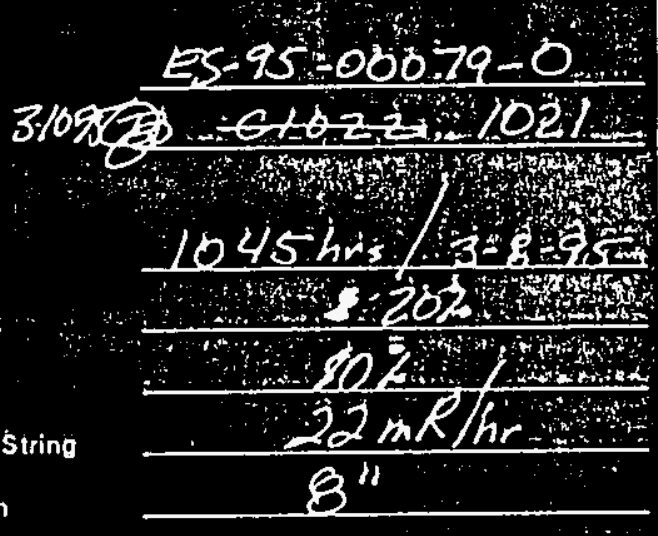

(9) INFORMATION (Include statoment of laboratory tosts to bo performod.)

\begin{abstract}
(10) Fold CERmonts
\end{abstract}
(32) Loboratory Commonts

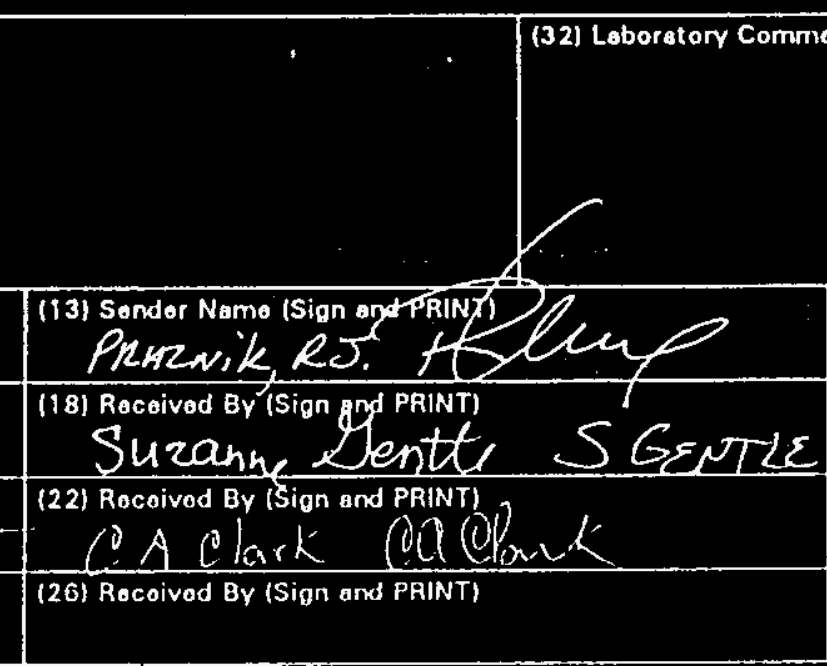

\section{QOPY}

monts

\begin{tabular}{|c|c|}
\hline $\begin{array}{l}\text { (14) Dato/Tims } \\
3 \cdot 10-95 / 14 z \text {; }\end{array}$ & $\begin{array}{l}\text { (15) Sondor Commonts } \\
\text { ETEXY } T \text { JNG iNTRCT }\end{array}$ \\
\hline $\begin{array}{l}\text { (19) Datofimg } \\
3-10-95 / 160\end{array}$ & (20) Rocoivar Commonts \\
\hline $\begin{array}{c}\text { (23) Datofime } \\
3-10 \cdot 955 \\
17.35\end{array}$ & (24) Rocoivor Commonts \\
\hline (27) Dato/Timo & (28) Rocaivor Commonts \\
\hline
\end{tabular}

(30) Soal Data Consistont with this Racord?

Shipmont No.
$\square$ Yos

प马Yōs 


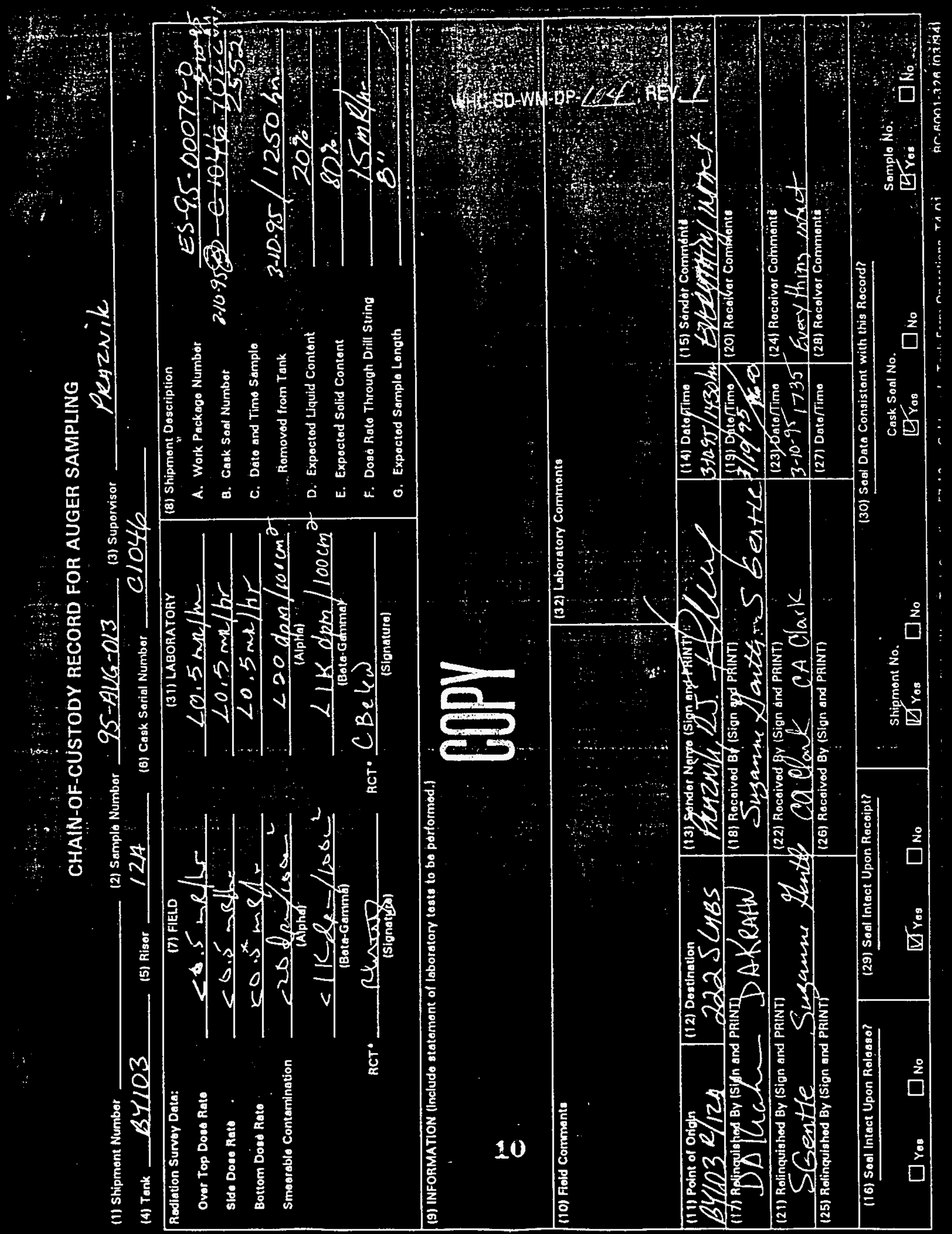


WHC-SD-WM-DP-104, REV . 1

PHOTOGRAPHS

11 
ts

THIS PAGE INTENTIONALLY

LEFT BLANK 


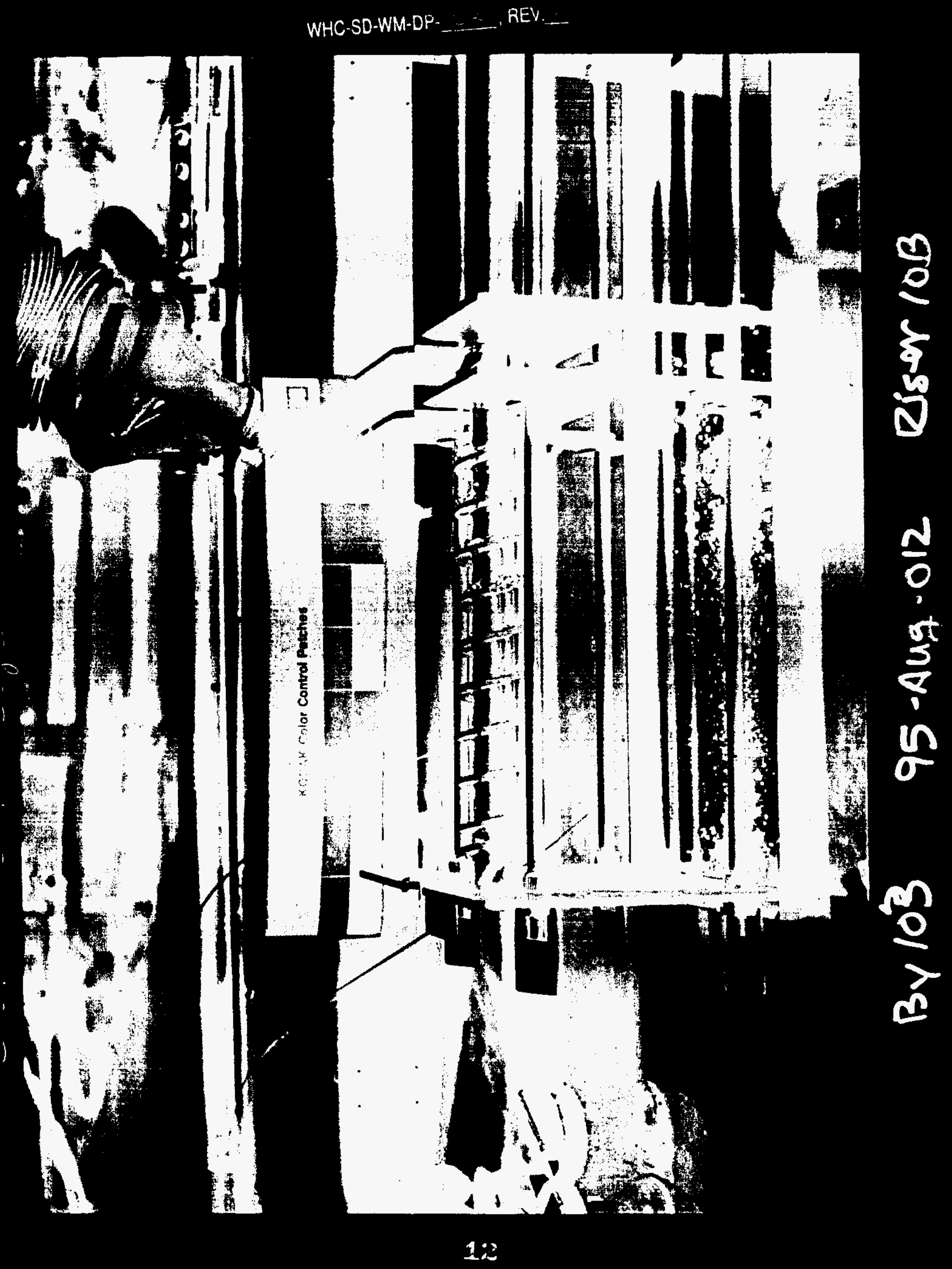


THIS PAGE INTENTIONALLY LEFT BLANK 
WHC-SD-WM-DP- 104 , REV _L

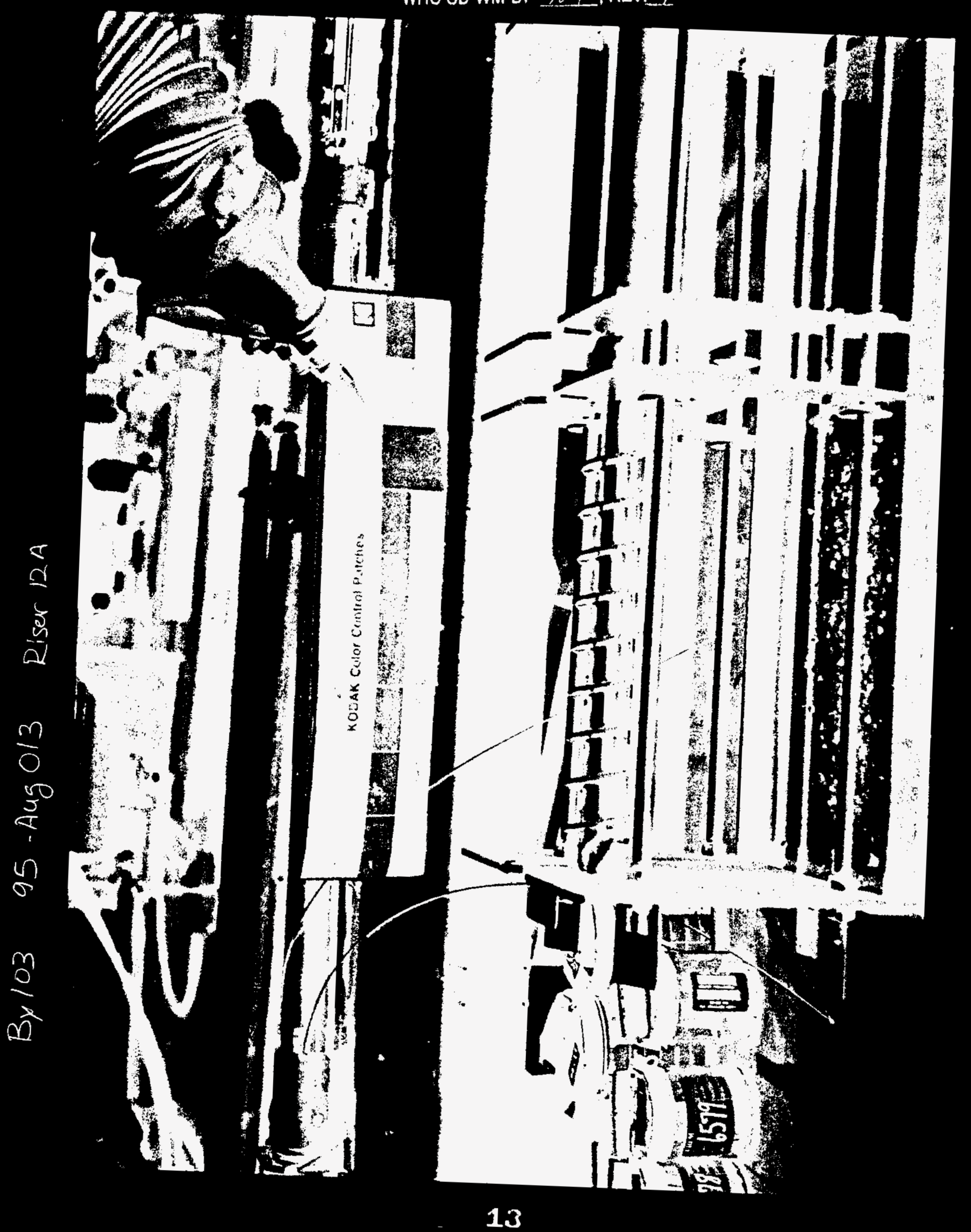




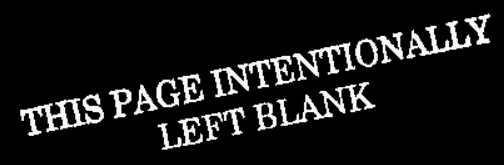


WHC-SD-WM-DP-104, REV. I

HOT CELL LOGBOOK 
TS PAGE INTENTIONALIY

THIS PAGE INT BLANK 
WHC-SD-WM-DP. 104, REV.

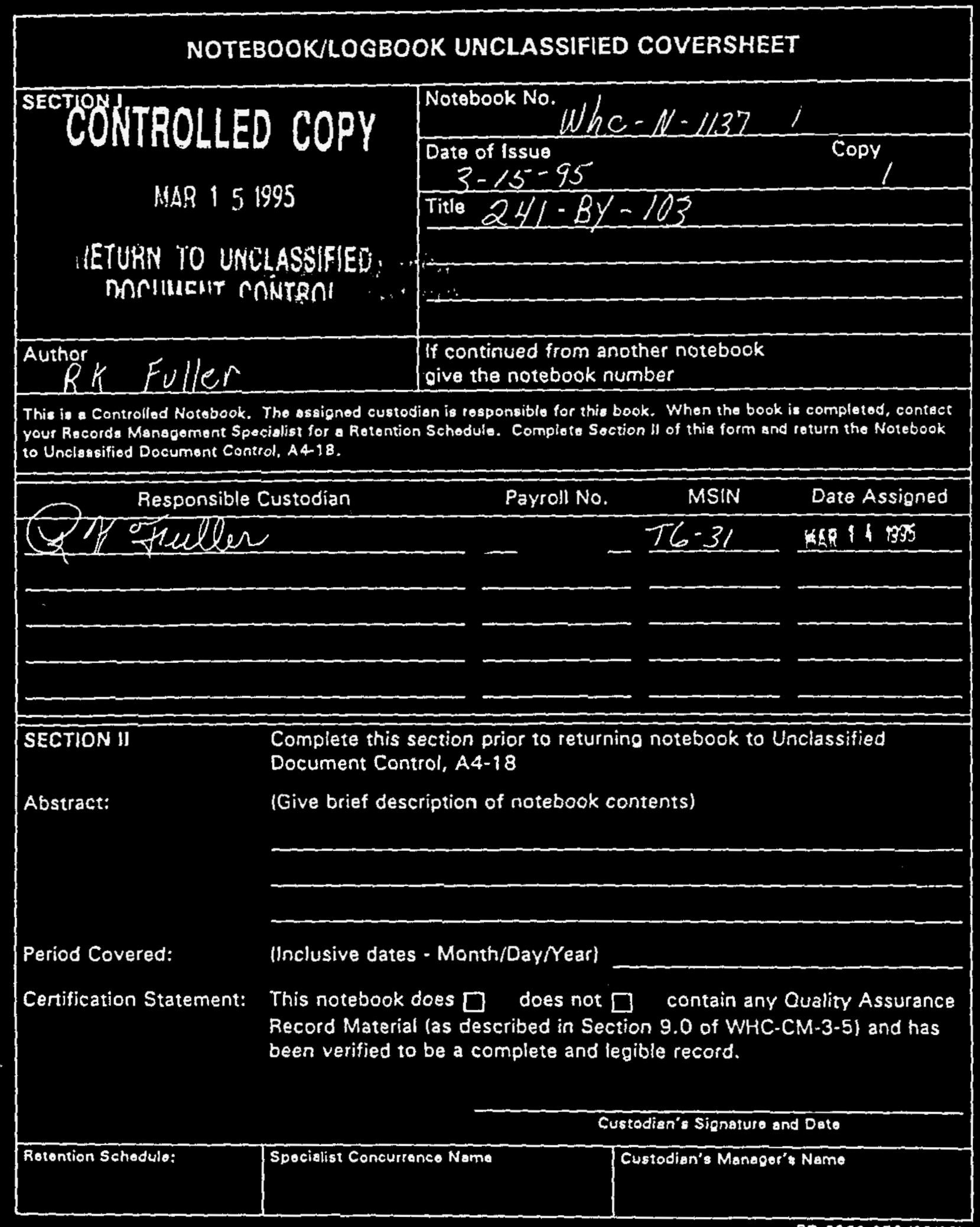


Table of Contents

\begin{tabular}{|c|c|c|}
\hline & Table of Contents & $i-5$ \\
\hline 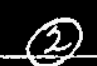 & By $103^{95-A U_{6}-012 \text { Riser } 10 B}$ & $6-9$ \\
\hline (3) & 95-AUG-013 Resen 12A & $10-13$ \\
\hline (4) & 95-Aug-012 Segl Reer 1013 Subrample & 14 \\
\hline (5) & 95-Rug-OL3 Seg 1 Riser $12 A$ Subsample & 15 \\
\hline
\end{tabular}


WHC.SO-WMON 104 , REV I

\author{
Pages 2 through 5 of Hot Cell Log Book \\ number WHC-N-1137 Copy l. were intentionally \\ left blank.
}


WHC-SD-WM-DP- 1.44, REV , I

6

3) Wow

$\therefore$ istis

(n)

$\because 4$

nitis

$\because$

ond

if

o N 30 , w

o 0

o 0 b
O

45 क 4 o

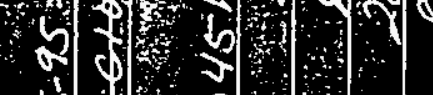
iv

(ivi)

m

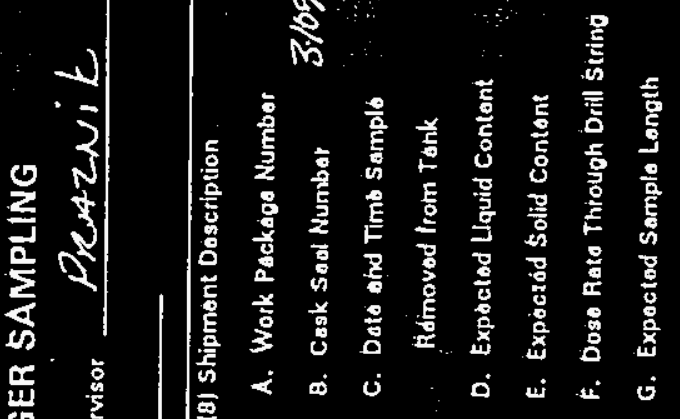

枈

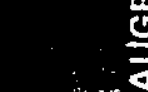

$\frac{10}{0}$

,

\%क

48

㟧

ar

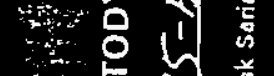

wis 0 o

, 0

14

$x_{0}$

$+\frac{1}{0}$

.

(n)

\section{........}



By 103
95-AUG-0/2
Risen 10B
$3 / 23 / 95$

Lahore worklist \#19 Lakcere number 5957000320 Videe * 2 BY tarm

$20 \mathrm{gos}=19.99 \mathrm{gms} \quad 500 \mathrm{gm}=499.98 \mathrm{gms}$

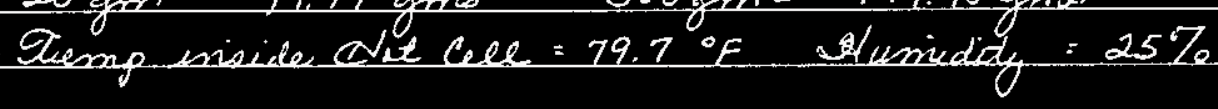

* No liner lequide (Dose Rate thru drill string = $22 \mathrm{~ms} / \mathrm{hr}$ )

Salice Description:

The total amount of selid motessil neconered uns 35.85 grams Here was no drainide hquie. Solid were very dry anol

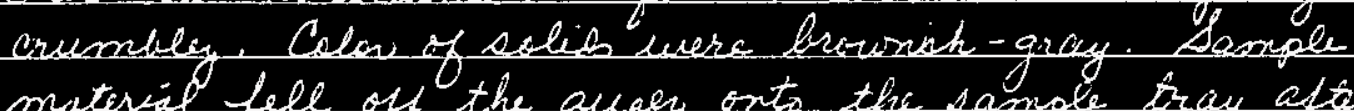
materiel fell of the auger onto the sample bray after the sleve was removed. No liquil was present. Wll the sample matersil appeared similar in Colsw and textere. Subomplet motrial into one fare. See subaromple information

BY 10395 -AUG-012 Solids:

- gir \# 6520 (125 mL jair aje)

-35.85 grame recovered.

gar \#6510
Final wt: 164.37 grams
Anitiel wt: 128.52 grams
net wt: $\quad 35.85$ grams

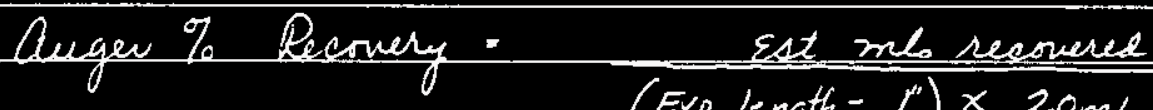

$\left(\right.$ Exp length $\left.-\mu^{\prime}\right) \times 20$ onch/in

$=\frac{35 \mathrm{~m} / \mathrm{s}}{7 \times 20} \times 100=$

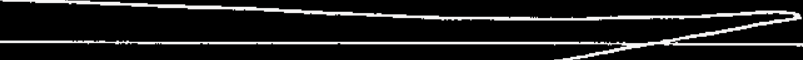

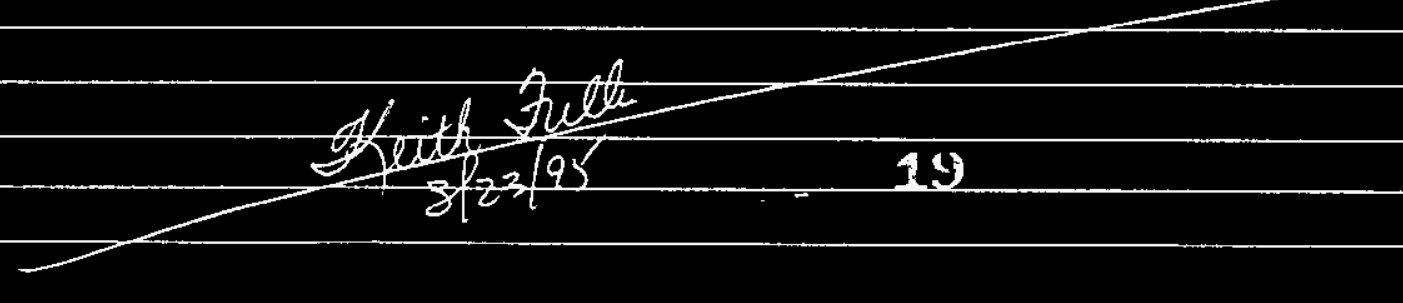


8

WHC-SD-WM-DF- 104, REV,

3/23/95 BY By 103 95-A46-012 Risen $10 B$

Aample reconery:

Estimatel tample velume $=35 \mathrm{~m} / \mathrm{s}$. This estimate is based on the grama of material collected and the material mat reconeres of the auges and vample tray. Araed on the 8 "eppected semple length, the persent secovery $=25 \%$ 


\section{WHC-SD-WM-DP 104, REV .}

BY 103 95-AUG-0/2 Riser $10 B$
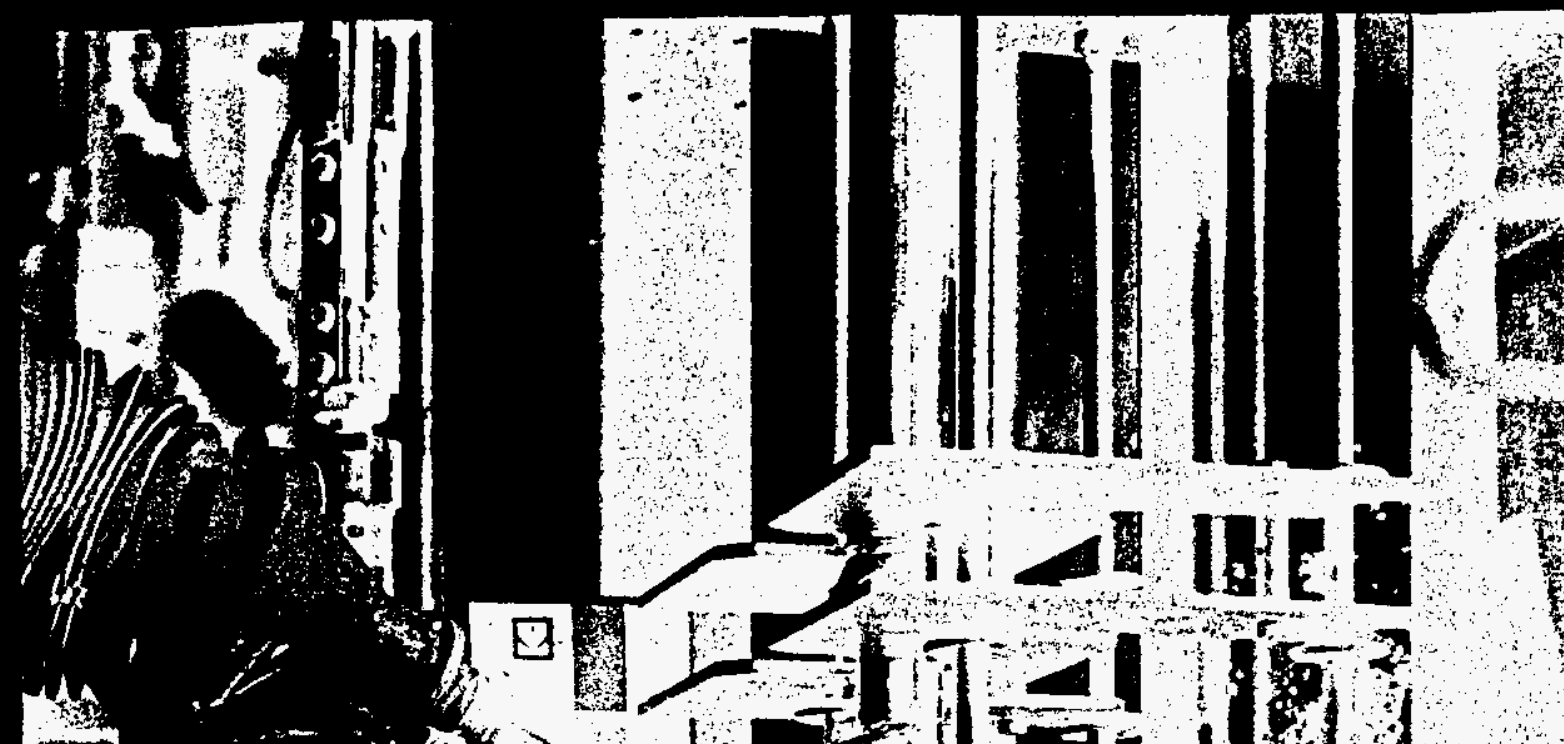

응

is 2 Ines

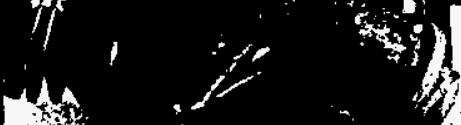

Q

$=1,15$
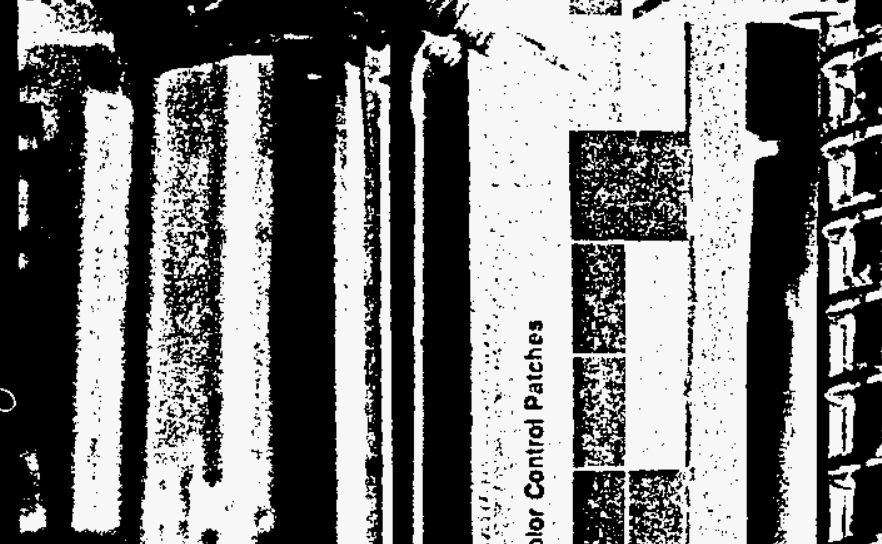

st

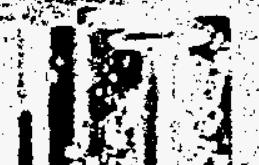

is

$\frac{1}{1+1}$
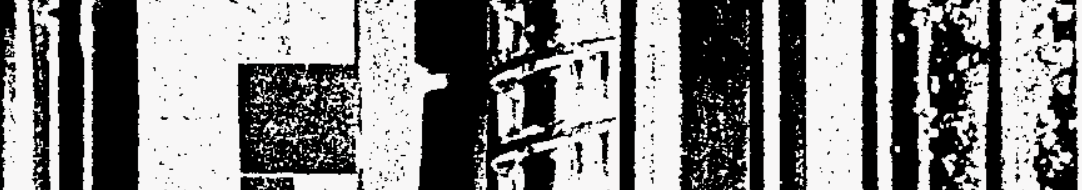

格
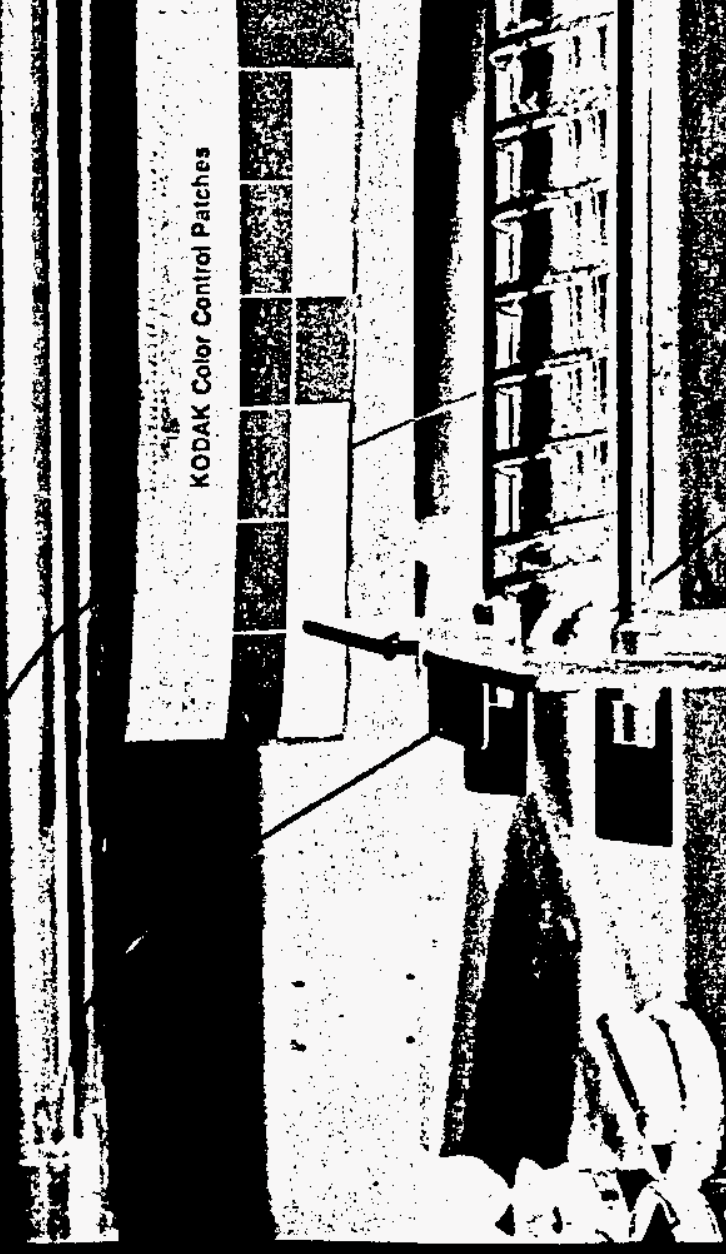

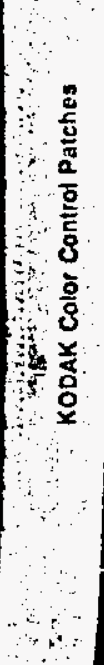
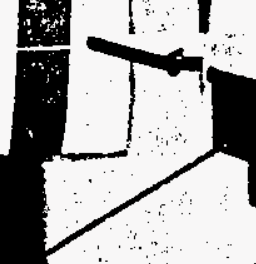

$=4$

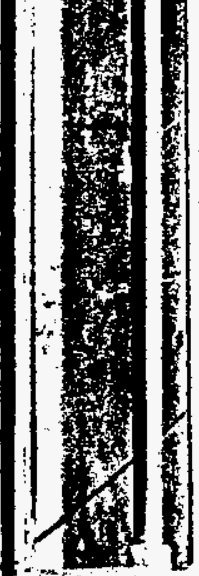

$P 1 \mathrm{~L}$

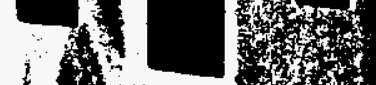

14

(n)

4

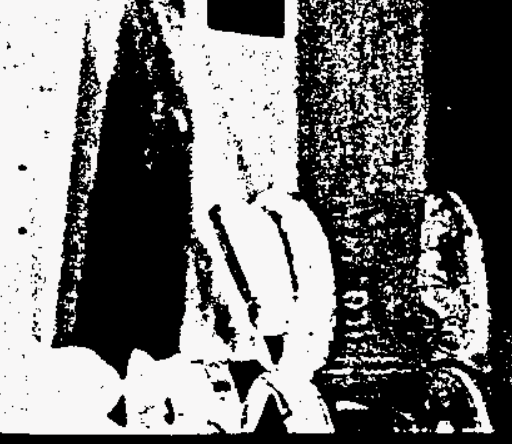

5

$\frac{1}{0}$

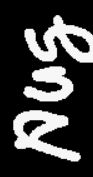

1

$\frac{M}{2}$ 
CHAIN-OF-CUSTODY RECORD FOR AUGER SAMPLING

(1) Shipmont Numbor (2) Samplo Number $95-A 1 C_{a}-0 / 3$ (3) Supervisor Peaznik

(4) Tank-3Y103 (5) Riser $\quad 124$ (8) Cask Serial Numbar $-\mathrm{C} / 04 / \mathrm{a}$

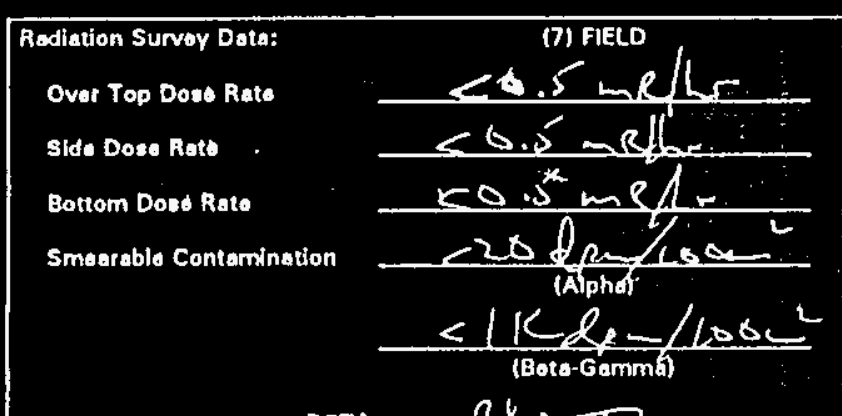

RCT• (Signatsis) -

$$
\begin{aligned}
& \text { (31) LABORATORY } \\
& 40.5 \mathrm{mn} / \mathrm{h} \\
& \therefore \quad 20.5 \mathrm{mr} / \mathrm{hr} \\
& \text { L.5me/thr } \\
& \angle 20 \mathrm{dpm} / 101 \mathrm{~cm}^{2} \\
& \frac{L 1 \mathrm{~K} \mathrm{dpm}_{\mathrm{pm}} / 100 \mathrm{~cm}}{\text { Boto-Gommat }} \\
& \text { CBelo }
\end{aligned}
$$

(8) Shipmont Description

A. Work Packogo Numbàr

B. Cask Soal Numbor

C. Dato and Time Sarmpio

Romoved from Tank

D. Expectod Liquid Contont

E. Expectod Solid Content

F. Dosó Rato Through Drill String

G. Expocted Somplo Longth

(9) INFORMATION finclude atotement of loboratory tosts to bo porformod.

\section{BOPY}

8

(10) Fold Commonts

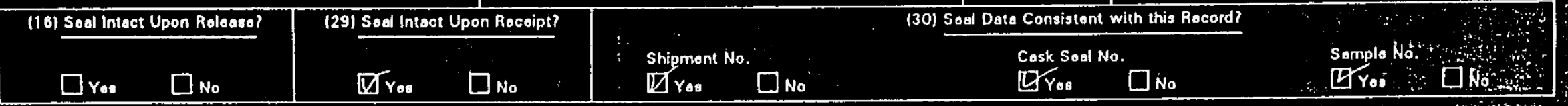




\section{WHC-SD-WM-DP-104, REY ! \\ $B y-10395$ AUG $0 / 3$ Riser $12 A(3-24.95)$}

$\angle A B C O R E$ WORKliST \#720. LABCCRE \#5957000321

VIDEO \#2 BY-FARM

$20 \mathrm{gm}=19.99 \mathrm{gmams} 500 \mathrm{gm}=499.98 \mathrm{~g} \mathrm{mms}$

Temp inside torcell $80.2^{\circ} \mathrm{F}$ Lumidity $20 \%$

No Liner LiQuid Dose RaTe thru Dall strina $(15 \mathrm{mR} / \mathrm{hr})$

Solids Description:o

Dry-CRUmBly Light yellow -Brown. Soms small clumber. sample fell off the augen. Very tialle somple remaind

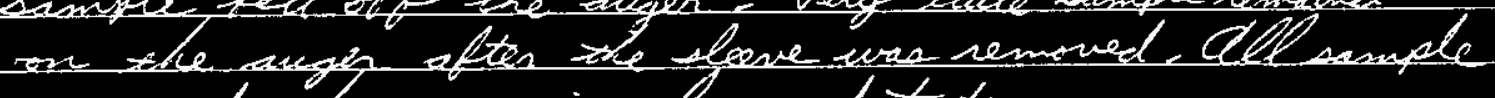
appeared the eme in clor end terture.

By 103 95-AvG-013 Solins

placed All selids in zhe amme jar. No crust, or segment type material olvenved.

JAR \#6578

JAR SizE $125 \mathrm{~m} \mid$

Final WT. $166.24 \mathrm{~g} 2 \mathrm{gms}$

INiTial wT 128.15 grams

Net wT. 38.09 grams

Sample Recovery

Estimated sample volume $=35$ mla. Tlis estemote is lessed on zhe orame of material sollected ond the sample tray, Based en the espected 8 inch sumple leargth, the percent recruery is apprimintely $25 \%$. 
12

WHC-SD-WM-DF /C LA, REV /

$$
\text { By } 103 \text { 95-AUG-013 Riser 12-A }
$$




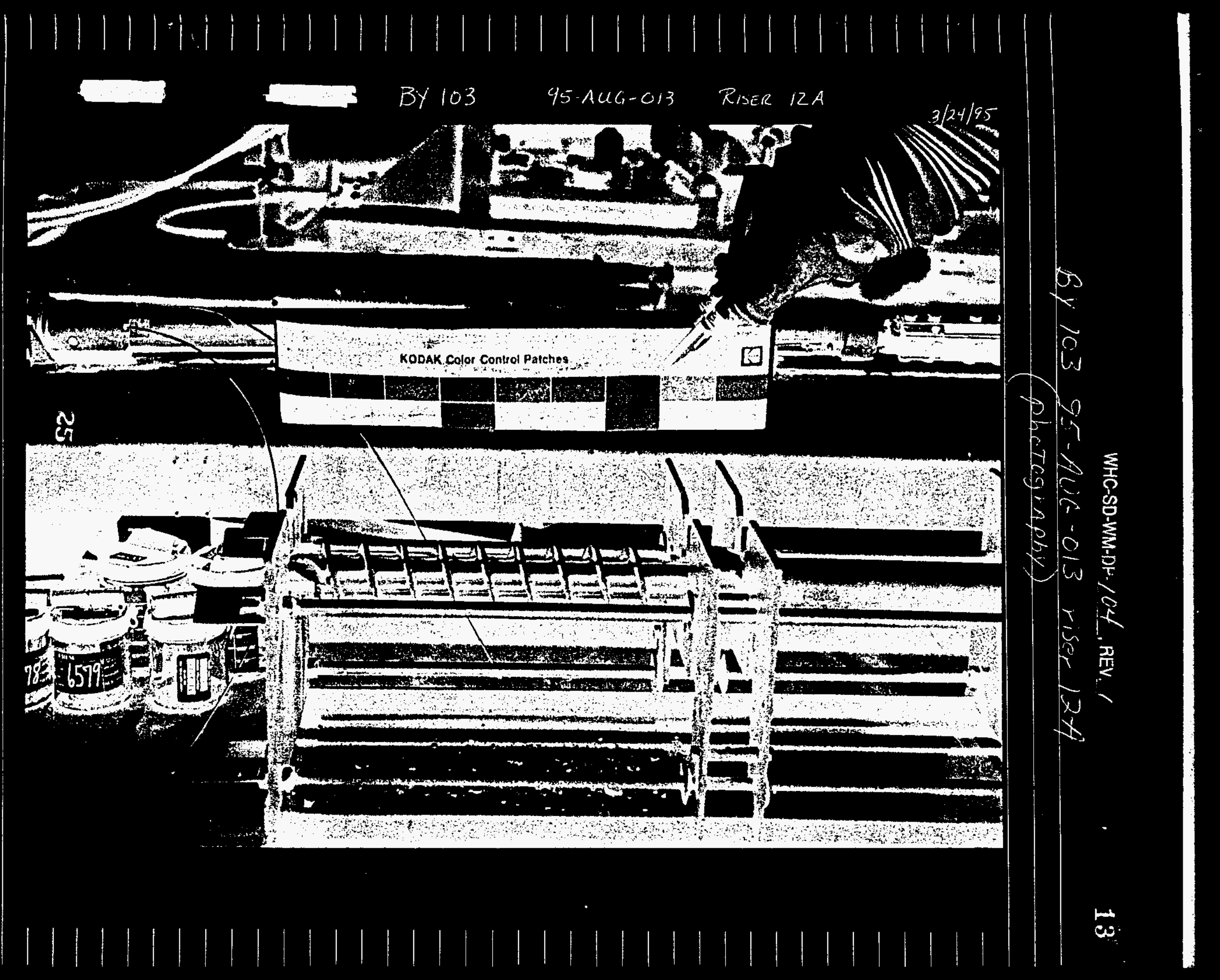




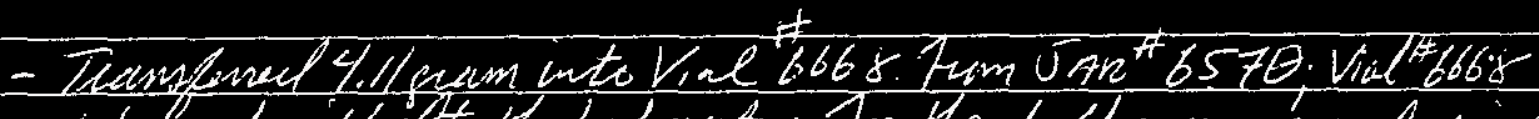

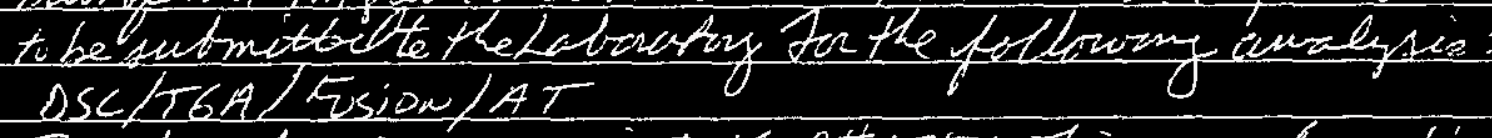

- Munfenel 28,17 mas into Vial 6756 , this was the entiv

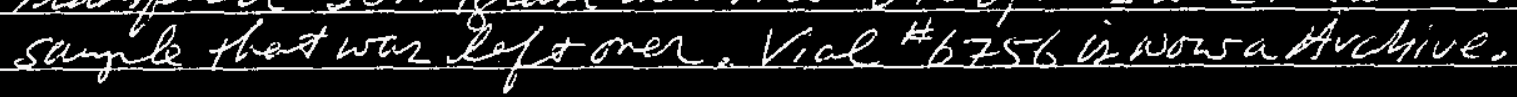

Lote: JAn 6570 is empty ancl has hean Destrogerl. 

BY 103 95ALS $\theta 13$ SEG $/ R-12 A$

$555 T 0 \theta 0511$

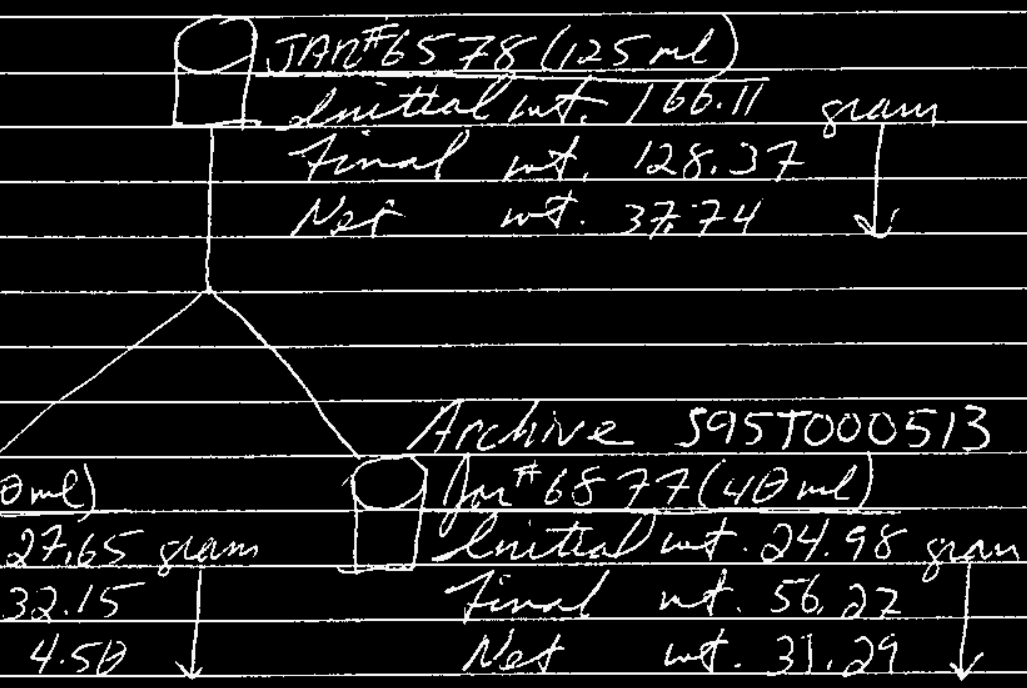

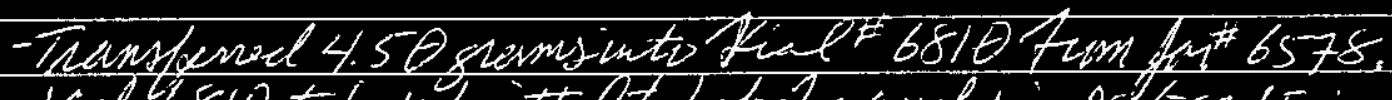

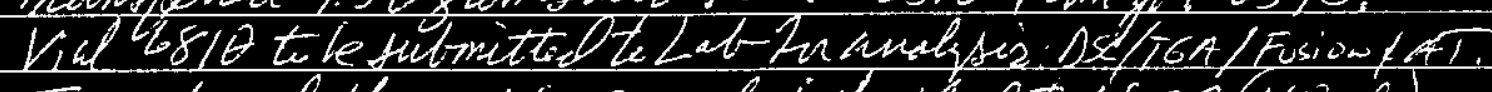

- Trangferrel the entire saysle intotial 6877 (40ul) An Avchiving.

Note: for $6578(125 \mathrm{ml}$ harbem Destinged. 
THIS PAGE INTENTIO

LEET BLARE 
WHC-SD-WM-DP-104, REV. 1

SAMPLE HANDLING

28 
THIS PAGE INTENTIONAILY LEFT BLANK 
Analyst: $\quad$ RK' $\quad$ Instrument: $\mathrm{BA000}$

Method: LO-160-103 Rev/Mod A-

Worklist Comment: BY-103 95-AUG-012 Extrusion
Book \#

WHC-SD-WM-DP-104, REV.

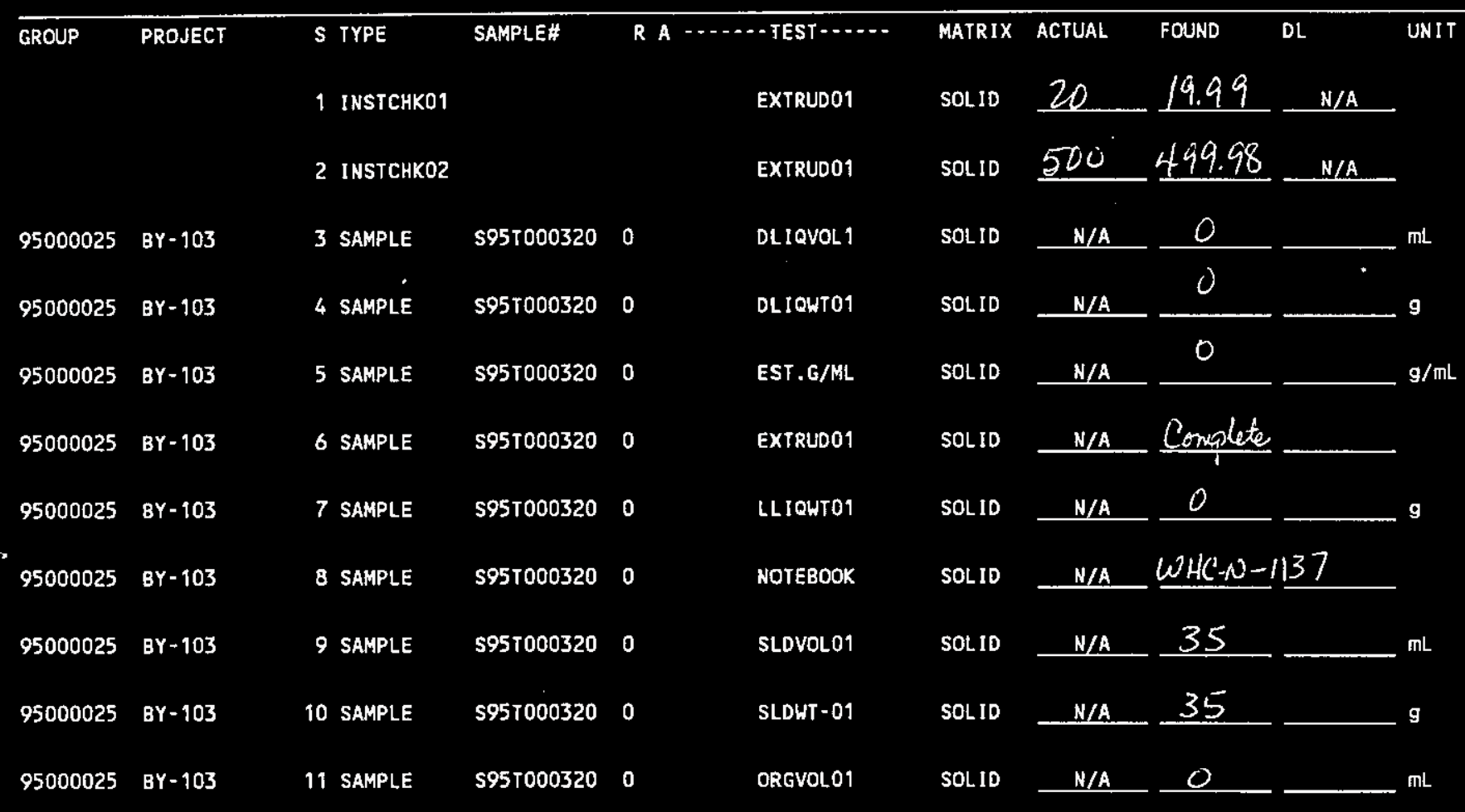

\section{Final page for worklist \# 719}

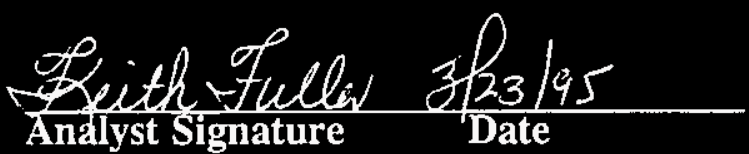

\section{Analyst Signature Date}

Data Entry Comments:

Units shown for QC (SPK \& STD) may not reflect the actual units. $D L=$ Detection Limit, $S=$ Worklist Slot Number, $R=$ Replicate Number, $A=$ Aliquot Code. 


\section{LABCORE Data Entry Template for Worklist\# 720}

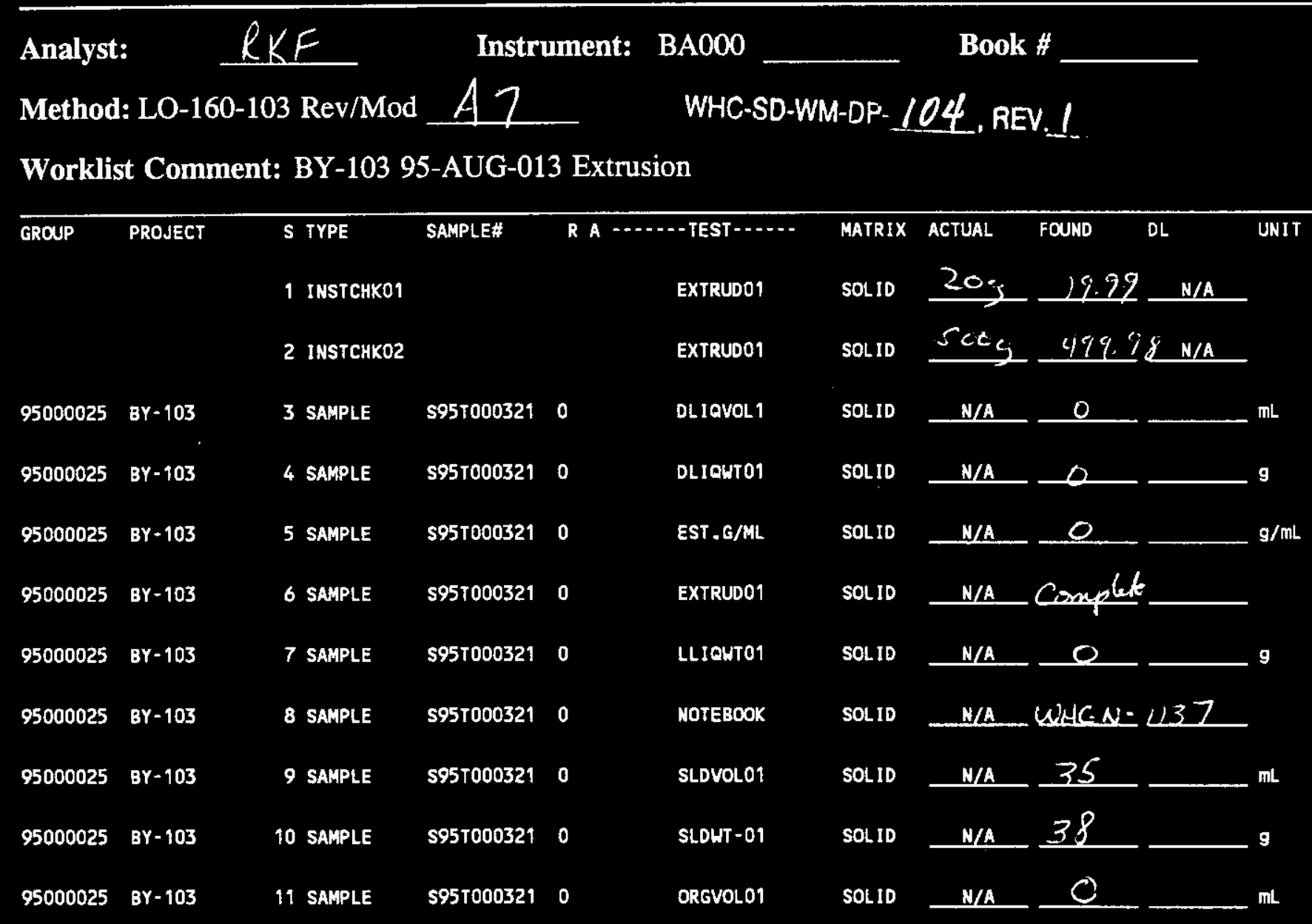

\section{Final page for worklist \# 720}

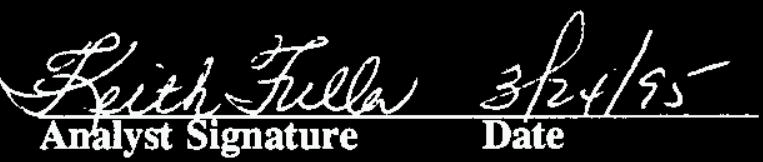

\section{Analyst Signature Date}

Data Entry Comments:

Units shown for $Q C$ (SPK \& STD) may not reflect the actual units. $D L=$ Detection Limit, $S=$ Worklist Slot Number, $R=$ Replicate Number, $A=$ Aliquot Code. 


\section{LABCORE Data Entry Template for Worklist\# 1070}

Analyst:

Instrument: BA000

Book $\# \underline{1}$ A

Method: LO-160-103 Rev/Mod A.?

Worklist Comment: BY-103 and U-204 Archive Samples

WHC-SD-WM-DP-104, REV, I

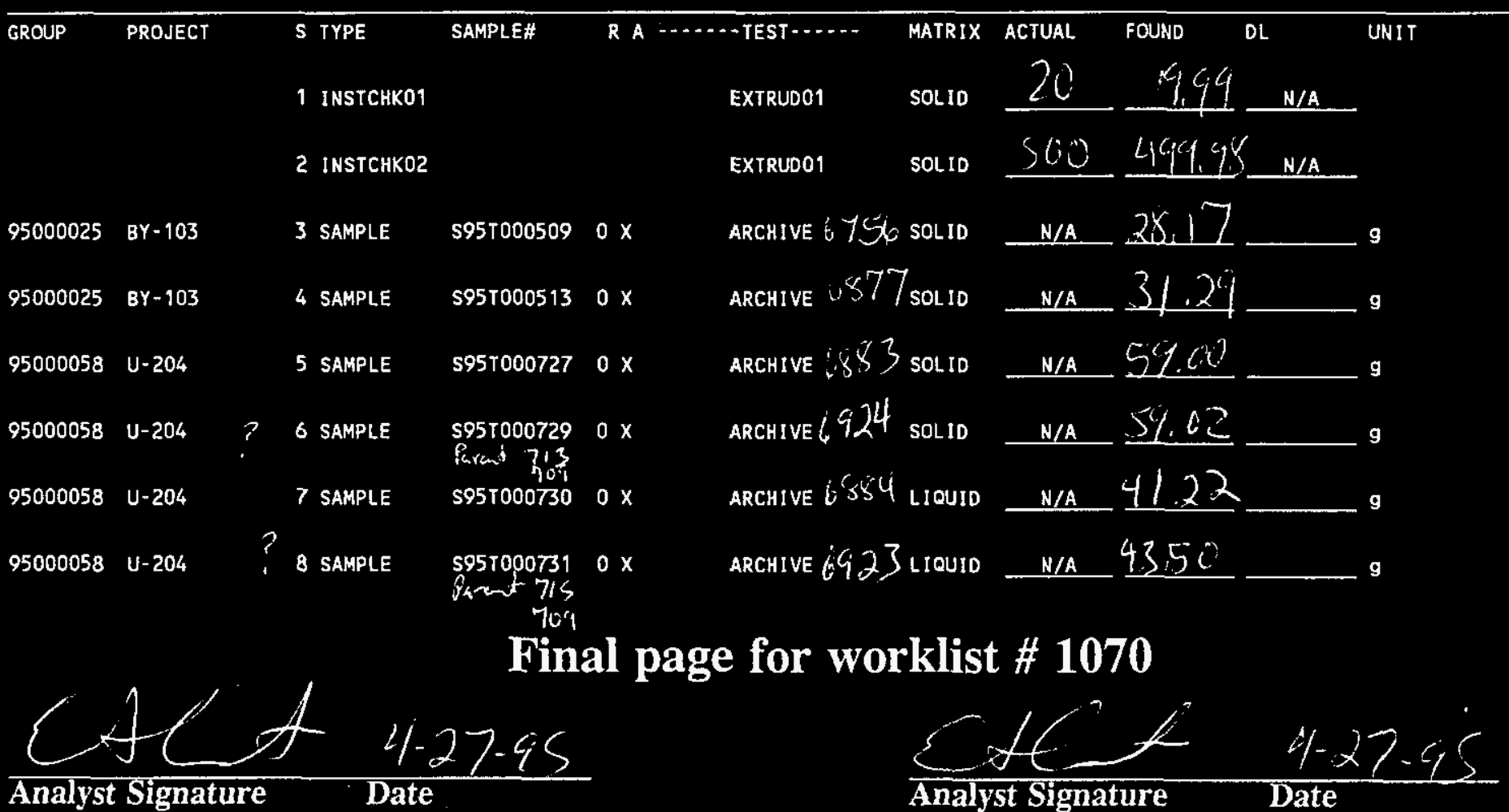

Data Entry Comments:

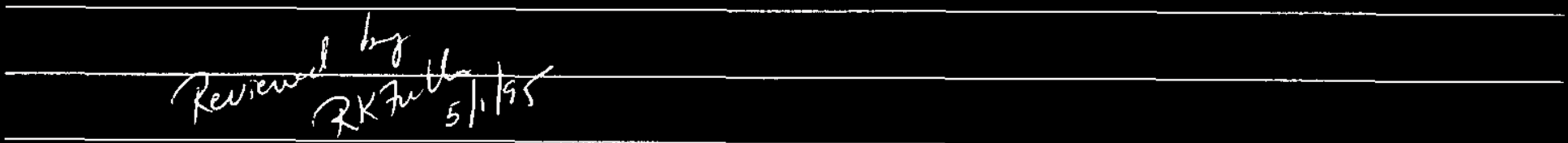

Units shown for $Q C$ (SPK \& STD) may not reflect the actual units. $D L=$ Detection Limit, $S=$ Worklist Slot Number, $R=$ Replicate Number, $A=$ Aliquot Code. 


\section{THIS PAGE INTENTIONALLY}

IEFT BLANK 
WHC-SD-WM-DP-104, REV. 1

SAMPLE PREPARATIONS

$3 \%$ 


\section{LABCORE Data Entry Template for Worklist\# 996}

Analyst:

Worklist Comment: BY-103 FUSION DIGEST - 507---> 508, 511--->512

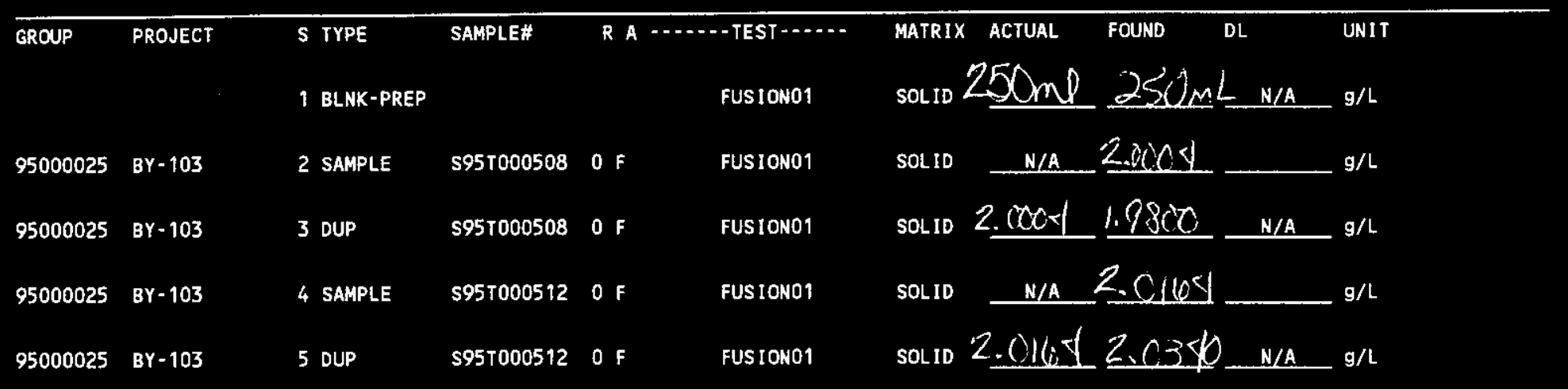

\section{Final page for worklist \# 996}

Vlesoullumphy $4-12-95$

$$
\begin{aligned}
& \begin{array}{l}
508 \\
\text { Sample } \frac{-5001 \mathrm{~g}}{.250 \mathrm{l}}=2.000 \mathrm{~g} / \mathrm{gl}
\end{array} \\
& 20 \mathrm{me} \mathrm{HCl} \\
& \text { Dup } \frac{.49509}{.250 l}-1.9800 \mathrm{~g} / \mathrm{l}
\end{aligned}
$$

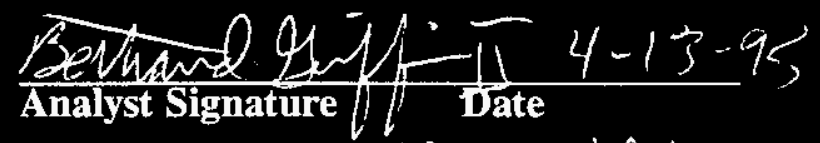
Analyst Signature

$5120.50410 \mathrm{~ms}$ HC. $\frac{512}{\operatorname{sam}} \frac{.50419}{.250 l}=2.016491 \%$

512
DUP

$$
\begin{gathered}
20 \mathrm{me} \mathrm{HCl} \\
\frac{.5085 \mathrm{q}}{.250 \mathrm{l}}=2.034 \mathrm{cq} / \mathrm{l}
\end{gathered}
$$

Datg Entry Comments:

grmput and dups were cleas. 190 oolids.

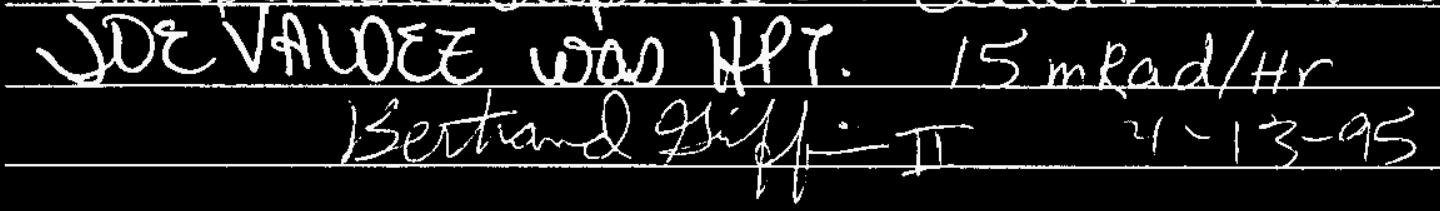

Units shown for $Q C$ (SPK \& STD) moy not reflect the actual units. $D L=$ Detection Limit, $S=$ Worklist Slot Number, $R=$ Replicate Number, $A=$ Aliquot Code. 
WHC-SD-WM-DP-104, REV, 1

RADIOCHEMICAL ANALYSES

34 
THIS PAGE INTENTIONALLY LEFT BLANK 


\section{LABCORE Data Entry Template for Worklist\# 1116}

\begin{tabular}{llll}
\hline Analyst: $\quad \operatorname{Im} V$ & Instrument: & $\mathrm{AB} 00 \frac{16}{27806}$ & Book \# 107852 \\
Method: LA-508-101 Rev/Mod & $\mathrm{D}-2$ & WB &
\end{tabular}

Worklist Comment: Determine sample size using Ludlum. Use $.100 \mathrm{~mL}$ A-SPK. SLF

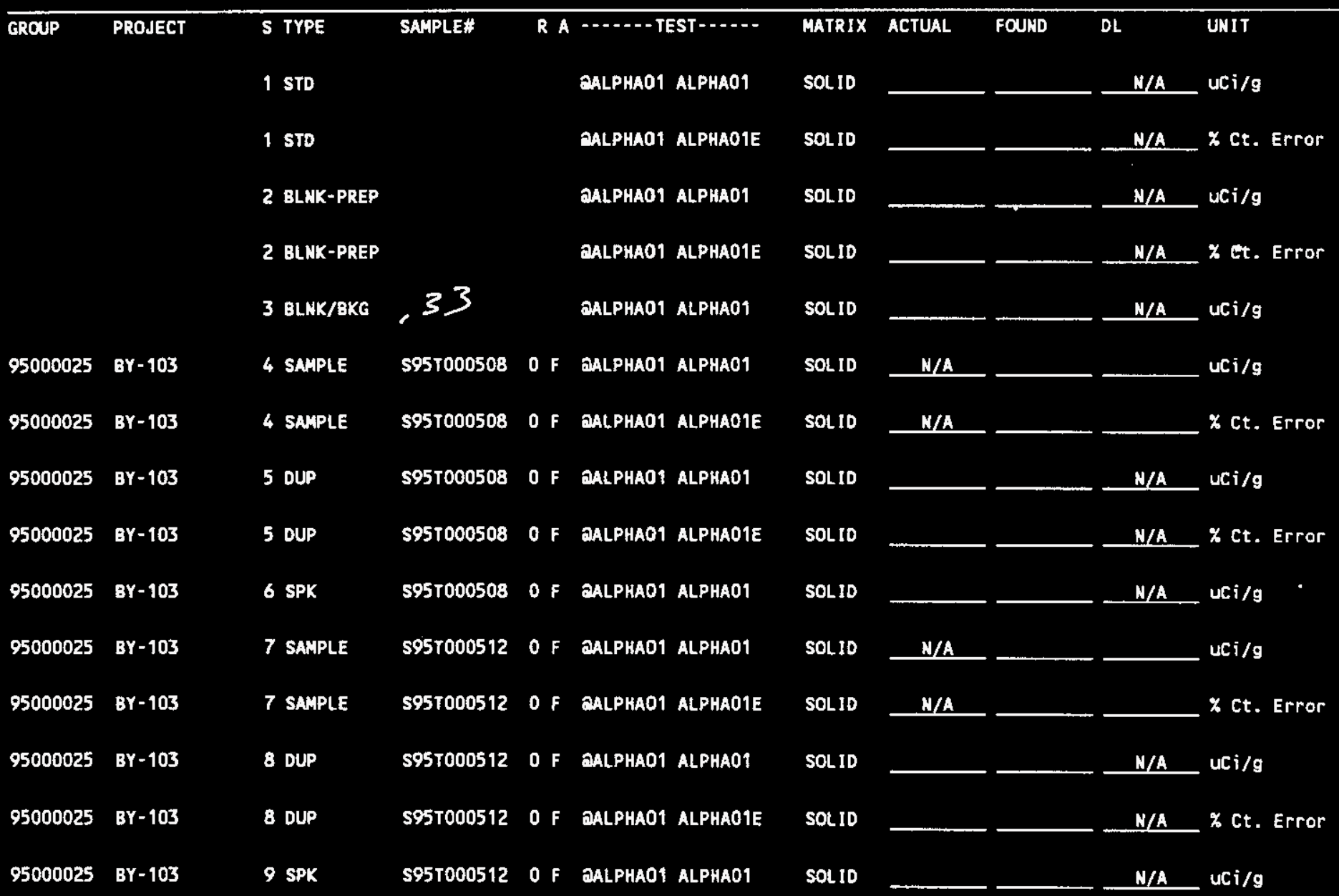

\section{Final page for worklist \# 1116}

doe mulansont $4-12,95$

Data Entry Comments:

SPK recoveries

workl.rt

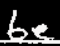

be out

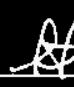

$4-18-95$

Units shown for $Q C$ (SPK \& STD) may not reflect the actual units. $D L=$ Detection Limit, $S=$ Worklist Slot Number, $R=$ Replicate Number, $A=$ Aliquot Code. 


\section{LABCORE Data Entry Template for Worklist\# 1116}

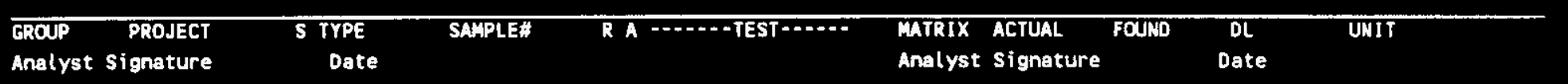

WHC-SD-WM-DP- 104 , REV.

Units shown for QC (SPK \& STD) may not reflect the actual units. $D L=$ Detection Limit, $S=$ Worklist Slot Number, $R=$ Replicate Number, $A=$ Aliquot Code. 


\begin{tabular}{|c|c|c|c|}
\hline \multicolumn{4}{|c|}{ AT : LA-508-101 (D-2) } \\
\hline \multicolumn{2}{|c|}{ LA-548-101 (A-3) } & STANDARD & REPLICATE \\
\hline 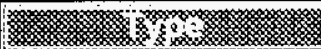 & DETECTOR NUMBER & 16 & 16 \\
\hline STAMDAR3. & DISH SIZE $\quad 1,2$, or 5 & 2 & 2 \\
\hline 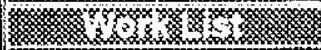 & TOTAL COUNTS & 4556 & 4702 \\
\hline 1116 . & COUNT TIME in MINUTES & 30 & 30 \\
\hline 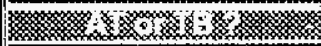 & BACKGROUND in cpm & 0.1 & 0.1 \\
\hline AT & SAMPLE SIZE in $\mathrm{mL}$ & 10,000 & 10.000 \\
\hline 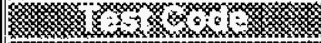 & DILUTION FACTOR & 1 & 1 \\
\hline 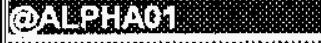 & DICEST DILUTION FACTOR & 1 & 1. \\
\hline 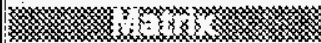 & EFFICIENCY FACTOR & 0.2104 & 0.2104 \\
\hline MQU1B & LC, Rmax, or Rs,(SAMPLE RATE) as APPROPRIATE & 151.767 & 156.633 \\
\hline 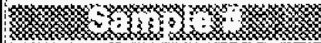 & Sample Concentration in $\mu \mathrm{C} i \mathrm{~L}$ & 3.25E-02 & BOOK\# \\
\hline WRKAST 116 & Replicate Concentration in $\mu \mathrm{CI}$ & $3.355-02$ & 107852 \\
\hline 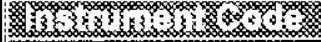 & & & \\
\hline WB27806 & Average Concentration in $\mu \mathrm{CilL}$ & 3.3013E-02 & \\
\hline$\frac{1}{3}$ & & & \\
\hline MMV & Rs (Sample Count Rate) & & \\
\hline (n) & $=\mathrm{Rs} * 1000 \mathrm{mLL}$ * DF*DDF / (EF & * SS * 22200000 & $\mathrm{~m} / \mu \mathrm{Ci})$ \\
\hline 041425 & ALPHA TOTAL $\mu \mathrm{Ci} / \mathrm{mL}=$ ALPHA TOTAL $\mu \mathrm{Cil} / 1000 \mathrm{~mL} /$ & & \\
\hline $20600 \mathrm{NW}$ & $\begin{array}{l}\text { Relative Counting Error }=\text { [ I(The Square Root of TC }+ \text { BKG } \\
\text { Detection Levels and Less Than Values are determined from P }\end{array}$ & $\begin{array}{l}\text { CT) } / \text { (TC - BKC } \\
\text { ocedure LA-508-0 }\end{array}$ & CT) $]=1.96 * 100$ \\
\hline
\end{tabular}

V RESULTS $v$

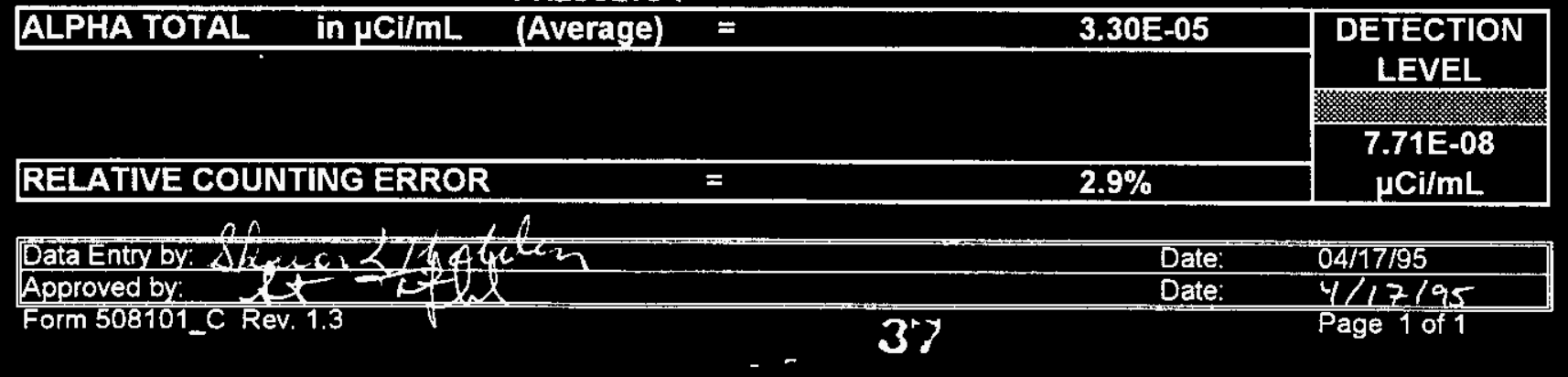




\begin{tabular}{|c|c|c|c|}
\hline \multicolumn{2}{|c|}{ AT : LA-508-101 (D-2) } & \multirow[b]{2}{*}{ BLANK } & \multirow[b]{2}{*}{ REPLICATE } \\
\hline LA- & $8-101(A-3)$ & & \\
\hline Yin & DETECTOR NUMBER & 16 & 16 \\
\hline BLANK & DISH SIZE $\quad 1,2$, or 5 & 2 & 2 \\
\hline menck & TOTAL COUNTS & 1. & 1 \\
\hline 1116 & COUNT TIME in MINUTES & 30 & 30 \\
\hline 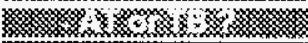 & BACKGROUND in cpm & 0.1 & 0.1 \\
\hline AT & SAMPLE SIZE in $\mathrm{mL}$ & 0.100 & 0.100 \\
\hline 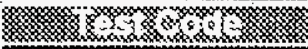 & DILUTION FACTOR & 1 & 1 \\
\hline OALPRAOD & DIGEST GRAMS of SOLIDSIL. & 20004 & 2.0004 \\
\hline 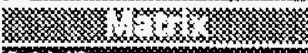 & EFFICIENCY FACTOR & 0.2104 & 0.2104 \\
\hline SOL:O & LC, Rmax, or Rs,(SAMPLE RATE) as APPROPRIATE & 0.135 & 0.135 \\
\hline . & Sample Concentration in $\mathrm{pCilg}$ & 1.44E-03 & BOOK\# \\
\hline $595 \mathrm{~T} 003$. & Replicate Concentration in $\mu \mathrm{Cllg}$ & $1.44 E-03$ & \\
\hline Wh & & & \\
\hline WB27806 & Maximum Concentration in $\mu \mathrm{Cilg}$ & 1.4419E-03 & \\
\hline ind & & & \\
\hline JMV & Rs (Sample Count Rate) & & \\
\hline . & ALPHA TOTAL $\mu \mathrm{Ci} / \mathrm{g}=$ Rs $* 1000 \mathrm{mLL} *$ DF $/(\mathrm{EFF} * \mathrm{SS}$ & " Dg/L * 2220000d & $\mathrm{m} / \mu \mathrm{Ci})$ \\
\hline 0464495 & & & \\
\hline argoopm & $\begin{array}{l}\text { Relative Counting Error }=[\mid(T \text { The Square Root of } \mathrm{TC}+\mathrm{BKG} \\
\text { Detection Levels and Less Than Values are determined from }\end{array}$ & CT) / (TC - BKG & CT)|l ${ }^{*} 1.96 * 10$ \\
\hline
\end{tabular}

v RESULTS v

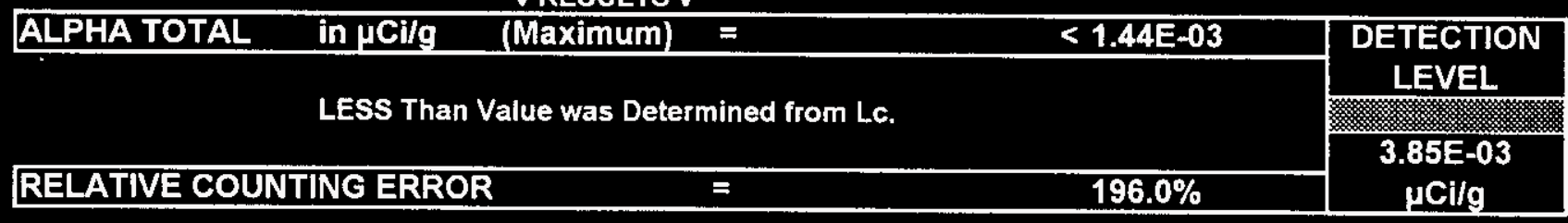

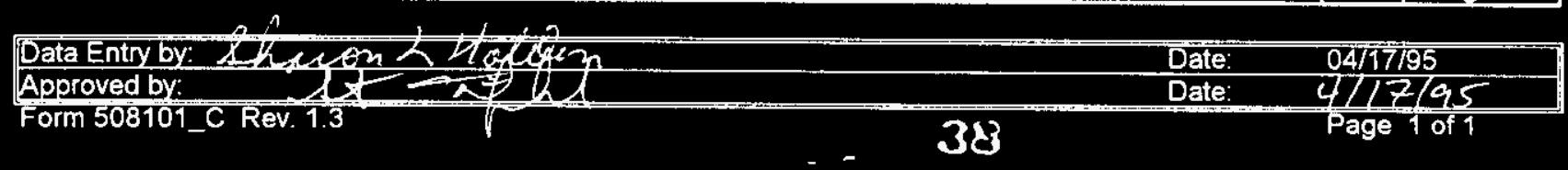


PLACE ANALYTICAL CARD IN BOX BELOW OR ATTACH TRAVELER WHC-SD-WM-DF. 104 , REV. 1

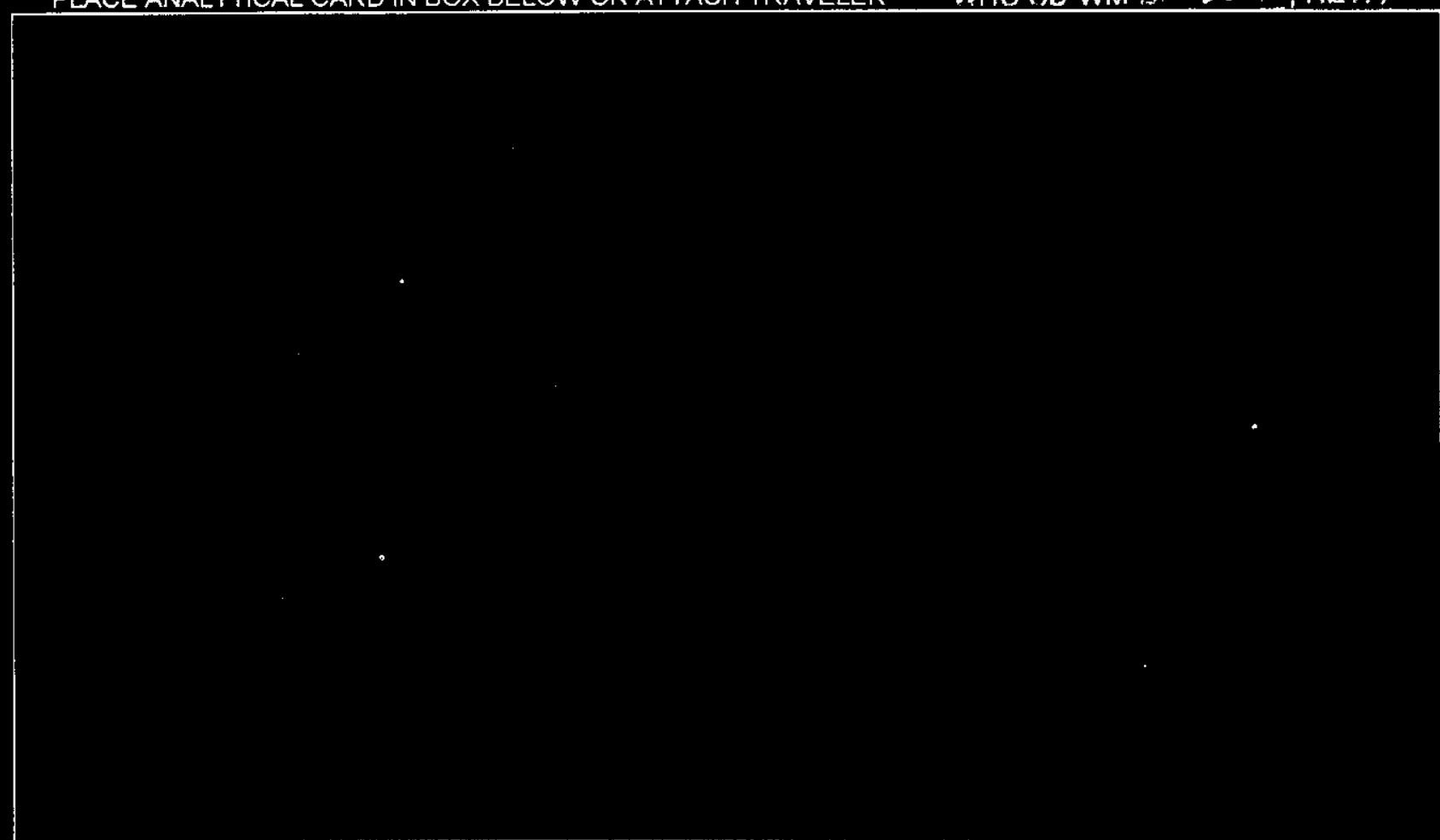

\begin{tabular}{|c|c|c|c|}
\hline \multicolumn{4}{|c|}{ AT : LA-508-101 (D-2) } \\
\hline \multicolumn{2}{|c|}{$L A-548-101(A-3)$} & \multirow[t]{2}{*}{ SAMPLE } & \multirow{2}{*}{ REPLICATE } \\
\hline 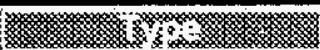 & DETECTOR NUMBER & & \\
\hline SAMPLE & DISH SIZE 1,2, or 5 & 2 & 2 \\
\hline ipen & TOTAL COUNTS & 67. & 72. \\
\hline $146.2 \times$ & COUNT TIME in MINUTES & 30. & 30 \\
\hline 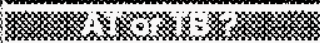 & BACKGROUND in cPm & 0.1 & 0.1 \\
\hline AT & SAMPLE SIZE in $\mathrm{mL}$ & 0.100 & 0.100 \\
\hline$x_{1}^{2}$ & DILUTION FACTOR & 1 & 1 \\
\hline OALPRAOT & DIGEST GRAMS of SOLIDSIL & 20004 & 2.0004 \\
\hline 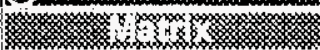 & EFFICIENCY FACTOR & 0.2104 & 0.2104 \\
\hline SOLIB & LC, Rmax, or Rs,(SAMPLE RATE) as APPROPRIATE & 2.133 & 2.300 \\
\hline 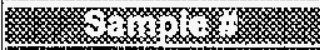 & Sample Concentration in $\mu \mathrm{Cilg}$ & $2.28 \mathrm{E}-02$ & BOOK\# \\
\hline S951508 & Replicate Concentration in $\mu$ Cilg & 2.46E-02 & \\
\hline W & & & \\
\hline WB278066 & Average Concentration in $\mu_{\text {Cilg }}$ & 2.3724E-02 & \\
\hline 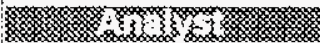 & & & \\
\hline IMV & Rs (Sample Count Rate) & & \\
\hline 9 & $=R s * 1000 \mathrm{~mL} / \mathrm{L} * \mathrm{DF} /(\mathrm{EFF} * \mathrm{~s} s$ & * Dg/L * 22200000 & $\mathrm{~m} / \mu \mathrm{Ci})$ \\
\hline Q44195: & & & \\
\hline Siston & Relative Counting Error $=[\mid(T$ The Square Root of TC + BKG & $\left.{ }^{*} \mathrm{CT}\right) /(\mathrm{TC}-\mathrm{BKG}$ & $\mathrm{CT}\rangle \mid] * 1.96 * 10$ \\
\hline $02000 \mathrm{RW}$ & Detection Levels and Less Than Values are determined from & ocedure LA-508-0 & \\
\hline
\end{tabular}

v RESULTS v

\begin{tabular}{|llc|c|}
\hline ALPHA TOTAL in $\mu \mathrm{Ci} / \mathrm{g}$ (Average) $=$ & $2.37 \mathrm{E}-02$ & $\begin{array}{c}\text { DETECTION } \\
\text { LEVEL }\end{array}$ \\
& & & $3.85 E-03$ \\
& & $25.6 \%$ & $\mathrm{CCi} / \mathrm{g}$ \\
\hline
\end{tabular}

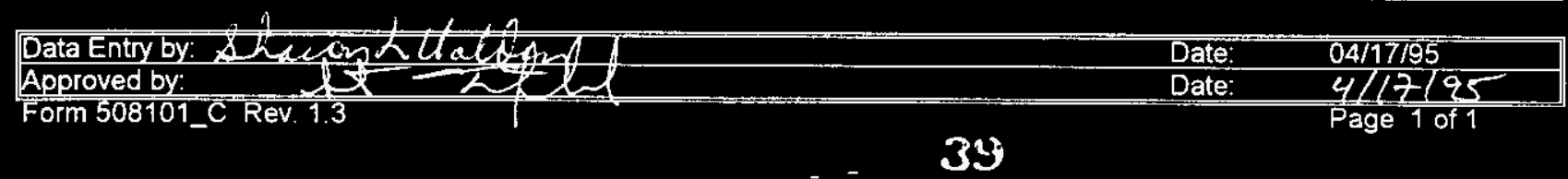




\begin{tabular}{|c|c|c|c|}
\hline \multicolumn{2}{|c|}{ AT : LA-508-101 (D-2) } & \multirow[b]{2}{*}{ SAMPLE } & \multirow[b]{2}{*}{ REPLICATE } \\
\hline \multicolumn{2}{|c|}{ LA-548-101 (A-3) } & & \\
\hline 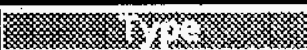 & DETECTOR NUMBER & 16 & 16 \\
\hline DUPUIATL & DISH SIZE $\quad 1,2$, or 5 & 2 & 2 \\
\hline moxys & TOTAL COUNTS & 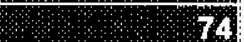 & 85 \\
\hline 1116 & COUNT TIME in MINUTES & 30 & 30 \\
\hline 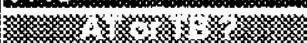 & BACKGROUND in cPm & 01 & 0.1 \\
\hline AT & SAMPLE SIZE in $\mathrm{mL}$ & 0.100 & 0.100 \\
\hline$\ldots$ & DILUTION FACTOR & 1 & 1 \\
\hline OALRRAO & DIGEST GRAMS of SOLIDSIL & 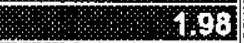 & 1.98 \\
\hline 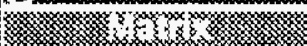 & EFFICIENCY FACTOR & 0.2104 & 0.2104 \\
\hline SOLD & Lc, Rmax, or Rs,(SAMPLE RATE) as APPROPRIATE & 2.367 & 2.733 \\
\hline ; ; inis & Sample Concentration in $\mu \mathrm{Ci} / \mathrm{g}$ & 2.56E-02 & BOOK\# \\
\hline 3951608 & Replicate Concentration in $\mu \mathrm{Ci} / \mathrm{g}$ & 2.96E-02 & \\
\hline 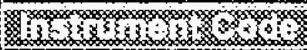 & & & \\
\hline WB27806 & Average Concentration in $\mathrm{PCilg}$ & $2.75735-02$ & \\
\hline 15 & & & \\
\hline MMV & Rs (Sample Count Rate) & & \\
\hline$\quad 1 \quad \ldots$ & $=R \mathrm{~s} * 1000 \mathrm{~mL} / \mathrm{L} * \mathrm{DF} /(\mathrm{EFF} * \mathrm{~S}$ & * Dg/L * 2220000 & $\mathrm{~m} / \mu \mathrm{Ci})$ \\
\hline 044495 & & & \\
\hline 12 & $\begin{array}{l}\text { Relative Counting Error }=[\text { [ (The Square Root of TC }+ \text { BK } \\
\text { Detection Levels and Less Than Values are determined from }\end{array}$ & CT) / (TC - BK & (CT) $] * 1.96 * 10$ \\
\hline
\end{tabular}

$\checkmark$ RESULTS $v$

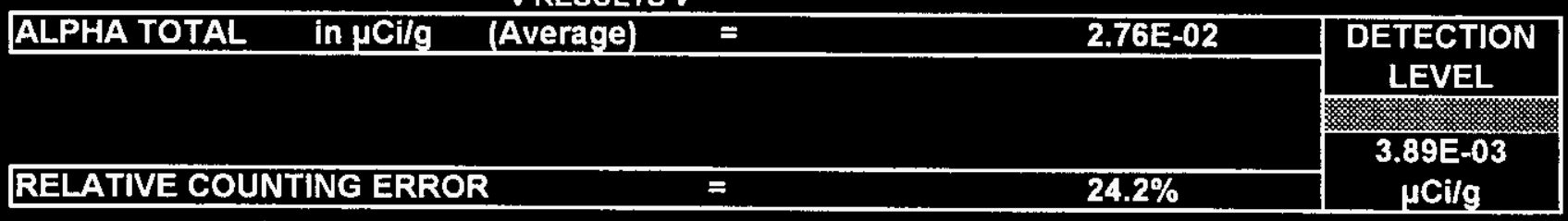

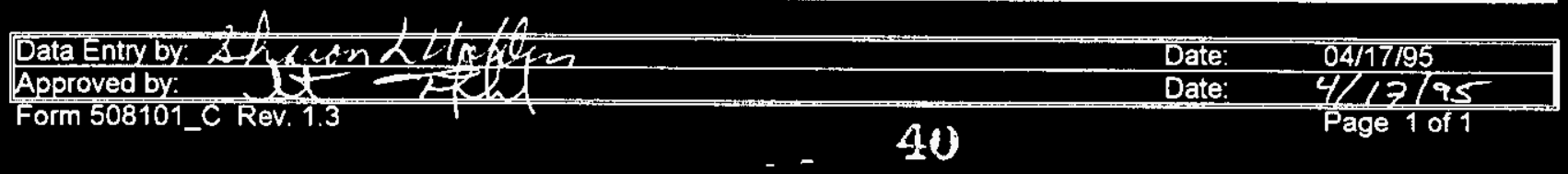




\section{AT : LA-508-101 (D-2)}

\section{LA-548-101 (A-3) SOLIDS}

\begin{tabular}{|c|c|c|c|}
\hline 10 & DETECTOR NUMBER & 16 & 16. \\
\hline SPIKE & DISH SIZE $\quad 1,2$, or 5 & 2 & 2 \\
\hline 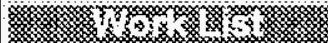 & TOTAL COUNTS & 47567 & 16758 \\
\hline I116 & COUNT TIME in MINUTES & 30 & 30 \\
\hline 1. & BACKGROUND in cpm & 0.1 & 0.1 \\
\hline$\Delta$ & SAMPLE VOLUME in $\mathrm{mL}$ & 0.100 & 0.100 \\
\hline 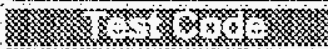 & SAMPLE DILUTION FACTOR (Spiked Vial) & 1 & 1 \\
\hline OAPHAOU & DIGEST GRAMS of SOLIDS/L & 2004 & 2.004 \\
\hline . & SPIKE VOLUME in mL & 0.100 & 0.100 \\
\hline SबIn & SPIKE DILUTION FACTOR & 11 & 1 \\
\hline mons & SPIKE VALUE in $\mu \mathrm{CI} / L$ & 36.372 & 36.372 \\
\hline $\mathrm{SgSTSO3}$ & INSTRUMENT EFFICIENCY FACTOR & 0.2104 & 0.2104 \\
\hline mis & SAMPLE + SPIKE $\mu \mathrm{Ci} / \mathrm{g}$ & $1.69 \mathrm{E}+01$ & $5.97 E+00$ \\
\hline WB27806 & AVERAGE or MAXIMUM $\mu$ Ci/g from FORM C & 2.37245202 & \\
\hline is & BOOK\# & 94843 & \\
\hline mive & \multirow{4}{*}{\multicolumn{3}{|c|}{$\begin{array}{l}\text { Rs (Sample Count Rate) }=(\text { TC /CT) - BKG } \\
\text { SAMPLE + SPIKE } \mu \mathrm{Cl} / \mathrm{g}=\mathrm{Rs} * 1000 \mathrm{mLL} * \mathrm{DF} /(\mathrm{EFF} \cdot \mathrm{SS} * \mathrm{Dg} / \mathrm{L} * 2220000 \mathrm{dpm} / \mu \mathrm{Ci}) \\
\text { PERCENT SPIKE RECOVERY }=(((\mathrm{S}+\mathrm{S} \mu \mathrm{Ci} / \mathrm{g}-\mathrm{SAMPLE} \mu \mathrm{Ci} / \mathrm{g}) *((\mathrm{SDF} / \mathrm{SVOl}))(\mathrm{DF} / \mathrm{SS} / \mathrm{Dg} / \mathrm{L}))) / \mathrm{SVal}) * 100\end{array}$}} \\
\hline 13 & & & \\
\hline 9476195. & & & \\
\hline$\frac{1200 \mathrm{PM}}{136}$ & & & \\
\hline
\end{tabular}

RESULT AVG. PERCENT SPIKE RECOVERY $=\quad 63.0 \%$

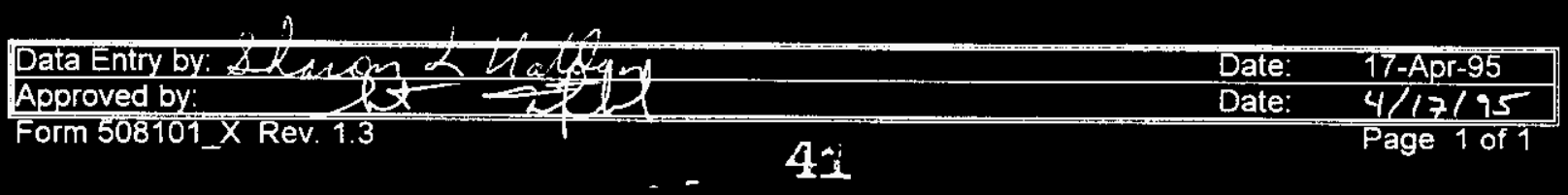




\begin{tabular}{|c|c|c|c|}
\hline \multicolumn{4}{|c|}{ AT : LA-508-101 (D-2) } \\
\hline \multicolumn{2}{|c|}{ LA-548-101 (A-3) } & \multirow[t]{2}{*}{ SAMPLE } & \multirow{2}{*}{ REPLICATE } \\
\hline man & DETECTOR NUMBER & & \\
\hline SAMPI: & DISH SIZE $\quad 1,2$, of 5 & 2. & 2 \\
\hline 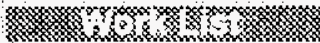 & TOTAL COUNTS & 13 & 15 : \\
\hline 116 & COUNT TIME in MINUTES & 30 & 30 \\
\hline 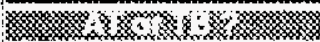 & BACKGROUND in cPm & 0.1 & 0.1 \\
\hline AT. & SAMPLE SIZE in $\mathrm{mL}$ & 6100: & 0.100 \\
\hline 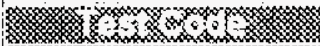 & DILUTION FACTOR & 1 & 1 \\
\hline (9)ALPAD & DIGEST GRAMS of SOLIDSIL & 2.0764 & 2.0164 \\
\hline 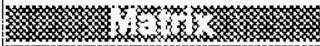 & EFFICIENCY FACTOR (EFF) & 0.2104 & 0.2104 \\
\hline SOLD & LC, Rmax, or Rs,(SAMPLE RATE) as APPROPRIATE & 0.333 & 0.400 \\
\hline 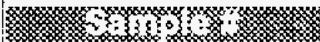 & Sample Concentration in $\mu \mathrm{CI} / \mathrm{g}$ & $3.54 E-03$ & BOOK\# \\
\hline 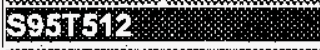 & Replicate Concentration in $\mathrm{HCi} / \mathrm{g}$ & 4.25E-03 & \\
\hline Mns & & & \\
\hline ME272006 : $:$ : & Average Concentration in $\mu \mathrm{Cilg}$ & $3.8931 E-03$ & \\
\hline $\min _{103}$ & & & \\
\hline IMV & Rs (Sample Count Rate) & & \\
\hline . & $=R s * 1000 \mathrm{mLL} * \mathrm{DF} /(\mathrm{EFF} * \mathrm{~S}$ & Dgh *2220000 & $\mathrm{m} / \mu \mathrm{Ci})$ \\
\hline 0.41495 & & & \\
\hline $01.00 \mathrm{PM}$ & $\begin{array}{l}\text { Relative Counting Error = [ I (The Square Root of TC }+ \text { BKC } \\
\text { Detection Levels and Less Than Values are determined from }\end{array}$ & $\left.{ }^{*} \mathrm{CT}\right)$ / (TC - BKG & CT) $\|] * 1.96 * 100$ \\
\hline
\end{tabular}

v RESULTS V

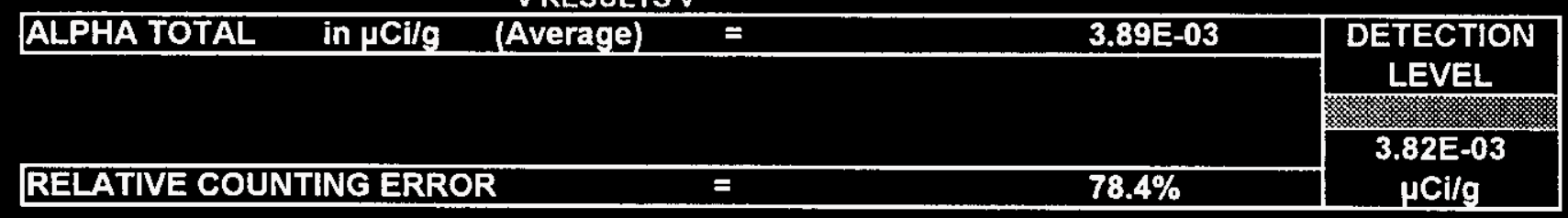

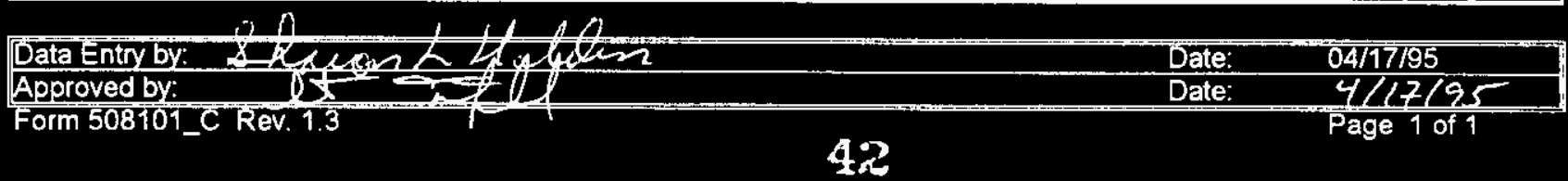


PLACE ANALYTICAL CARD IN BOX BELOW OR ATTACH TRAVELER WHC-SD-WM-DF-10 4 , REV,

\section{AT : LA-508-101 (D-2)}

\section{LA-548-101 (A-3) SOLIDS}

\begin{tabular}{|c|c|c|c|}
\hline \multicolumn{2}{|c|}{$L A-548-101(A-3)$} & SAMPLE & REPLICATE \\
\hline 10 & DETECTOR NUMBER & 16 & 16 \\
\hline DURLICATE & DISH SIZE 1,2 , or 5 & 2 & 2 \\
\hline 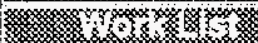 & TOTAL COUNTS & 9 & 25 \\
\hline $1116=280$ & COUNT TIME in MINUTES & 30 & 30 \\
\hline 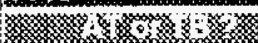 & BACKGROUND in cpm & 0.1 & 0.1 \\
\hline AT & SAMPLE SIZE in $\mathrm{mL}$ & 0.100 & 0.100 \\
\hline 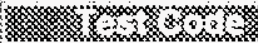 & DILUTION FACTOR & 1 & 1 \\
\hline OALPAOT & DIGEST GRAMS of SOLIDSIL & 2.034 & 2.034 \\
\hline (n) & EFFICIENCY FACTOR & 0.2104 & 0.2104 \\
\hline SOLD & LC, Rmax, or Rs,(SAMPLE RATE) as APPROPRIATE & 0.200 & 0.733 \\
\hline (n) & Sample Concentration in $\mu \mathrm{Ci} / \mathrm{g}$ & 2.11E-03 & BOOK\# \\
\hline S951512 & Replicate Concentration in $\mu \mathrm{Cilg}$ & 7.72E-03 & \\
\hline
\end{tabular}

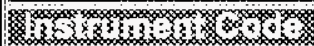

WB227806:

Average Concentration in $\mu \mathrm{Ci} / \mathrm{g}$

4.9120E-03

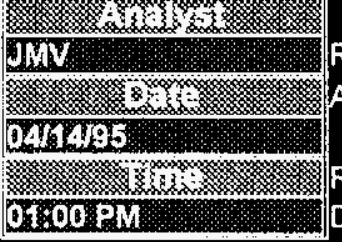

Rs (Sample Count Rate) $=(T C / C T)-B K G$

ALPHA TOTAL $\mu \mathrm{Ci} / \mathrm{g}=\mathrm{Rs} * 1000 \mathrm{mLL}$ * DF / (EFF * SS * Dg/L * 2220000dpm/ $\mathrm{CCi}$ )

Relative Counting Error $=\left[\right.$ [ (The Square Root of TC + BKG * CT) $/\left(T C-B K G{ }^{*} \text { CT) } \mid\right]^{*} 1.96 * 100$ Detection Levels and Less Than Values are determined from Procedure LA-508-002.

V RESULTS $y$

\begin{tabular}{|lll|c|}
\hline ALPHA TOTAL in $\mu \mathrm{Ci} / \mathrm{g}$ (Average) $=$ & $4.91 \mathrm{E}-03$ & $\begin{array}{c}\text { DETECTION } \\
\text { LEVEL }\end{array}$ \\
& & & \\
\hline RELATIVE COUNTING ERROR & $=$ & $113.2 \%$ & $3.79 E-03$ \\
$\mu \mathrm{Ci} / \mathrm{g}$
\end{tabular}

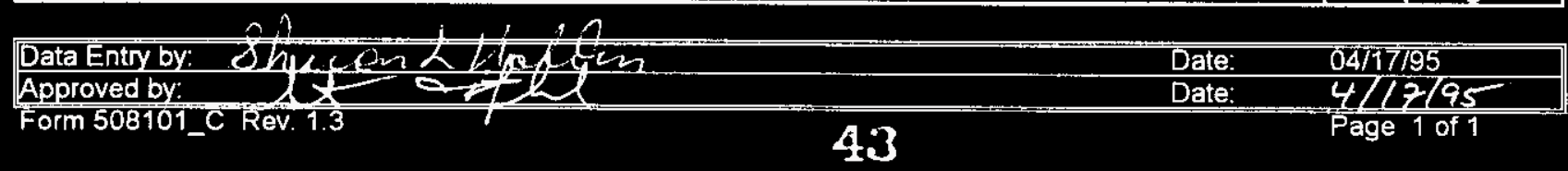


PLACE ANALYTICAL CARD IN BOX BELOW OR ATTACH TRAVELER WHC-SD-WM-DF- 104.4 , HEV: $/$

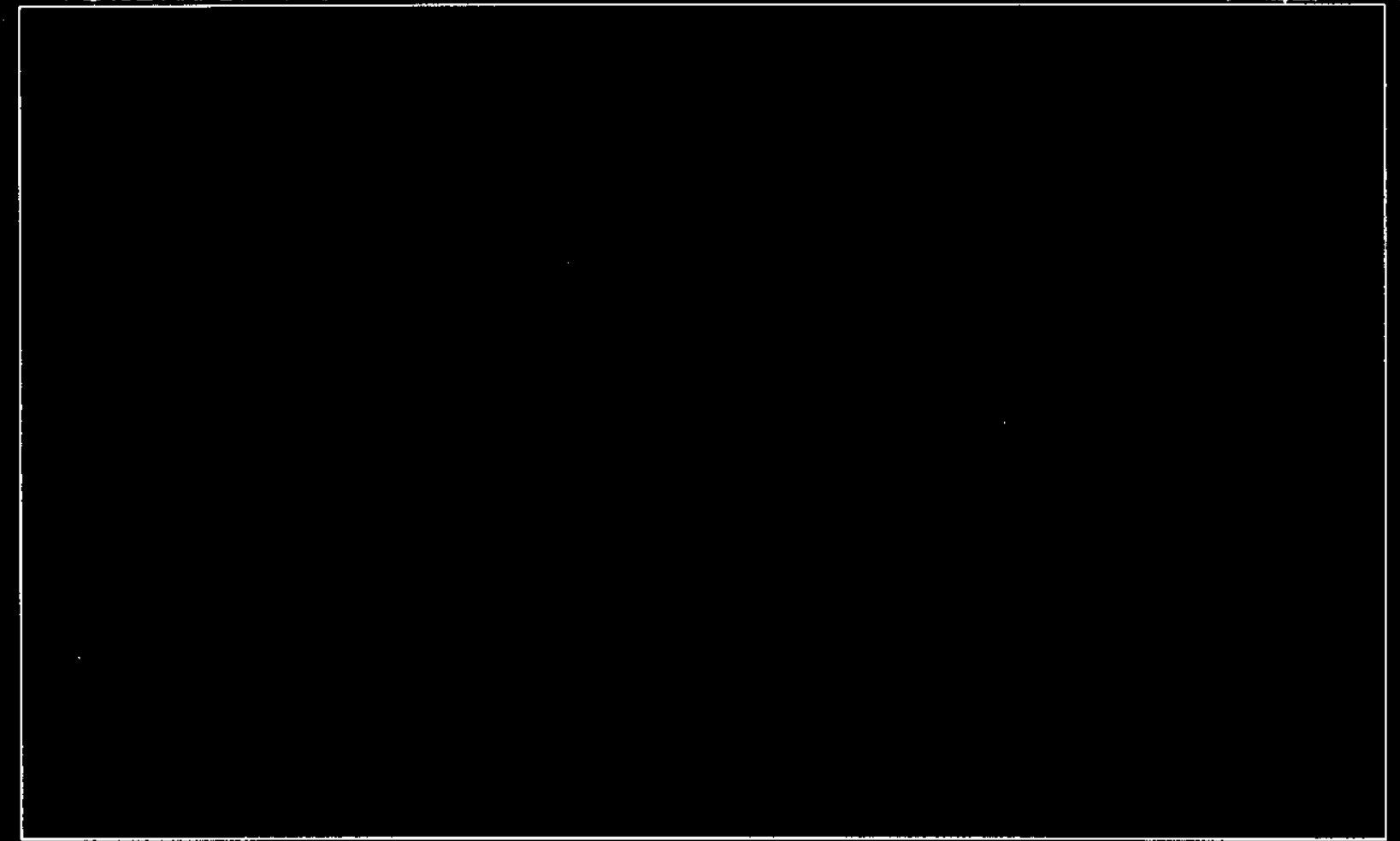

AT : LA-508-101 (D-2)

\begin{tabular}{|c|c|c|c|}
\hline 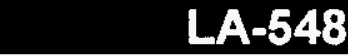 & SOLIDS & SPIKE & REPLICATE \\
\hline 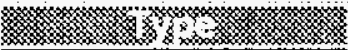 & DETECTOR NUMBER & 16 & 16 \\
\hline SPIKE & DISH SIZE $\quad 1,2$, or 5 & 2 & 2 \\
\hline - & TOTAL COUNTS & 4277 & 45201 \\
\hline (1116- & COUNT TIME in MINUTES & 30 & 30 \\
\hline 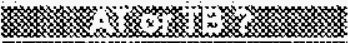 & BACKGROUND in cpm & 01 & 0.1 \\
\hline 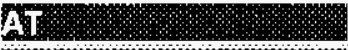 & SAMPLE VOLUME in $\mathrm{mL}$ & 0.100 & 0.100 \\
\hline$\times x$ & SAMPLE DILUTION FACTOR & 1 & 1 \\
\hline OALPRAOI & DIGEST GRAMS of SOLIDS/L & 20164 & 2.0164 \\
\hline mis & SPIKE VOLUME in $\mathrm{mL}$ & 0.100 & 0.100 \\
\hline SOLD & SPIKE DILUTION FACTOR & 1 & 1 \\
\hline W & SPIKE VALUE in $\mu \mathrm{CI} / \mathrm{L}$ & 36.372 & 36.372 \\
\hline S95ISI 2 . . . . & INSTRUMENT EFFICIENCY FACTOR & 0.2104 & 0.2104 \\
\hline 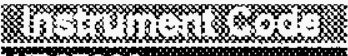 & SAMPLE + SPIKE $\mu \mathrm{Ci} / \mathrm{g}$ & $1.51 \mathrm{E}+01$ & $1.60 \mathrm{E}+01$ \\
\hline W627806 & AVERAGE or MAXIMUM $\mu \mathrm{Ci} / \mathrm{g}$ from FORM $\mathrm{C}$ & 38991E-03 & \\
\hline siands & BOOK\# & 94843 & \\
\hline GMV & \multirow{5}{*}{\multicolumn{3}{|c|}{ 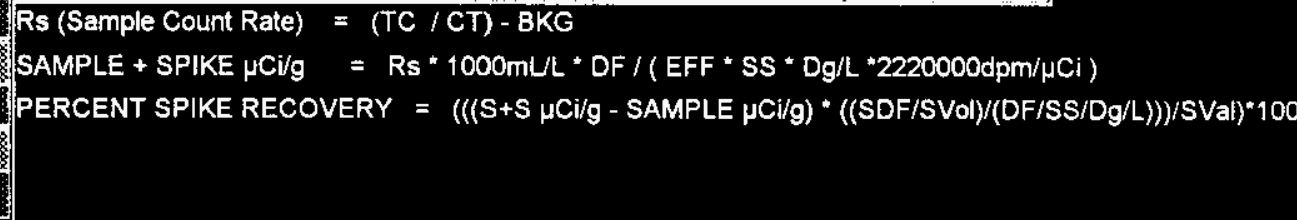 }} \\
\hline . & & & \\
\hline 0474295 & & & \\
\hline 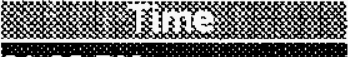 & & & \\
\hline 0100 P & & & \\
\hline
\end{tabular}

RESULT AVG. PERCENT SPIKE RECOVERY $=86.3 \%$

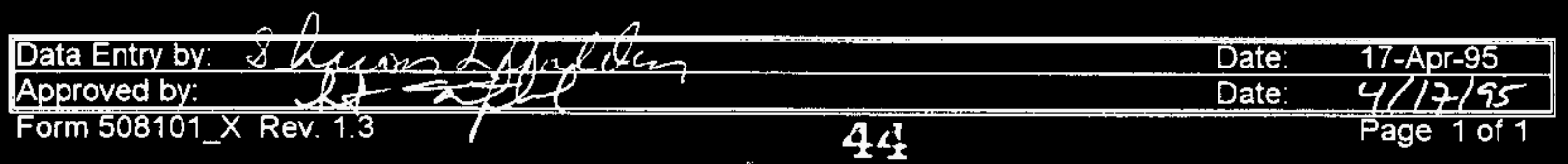




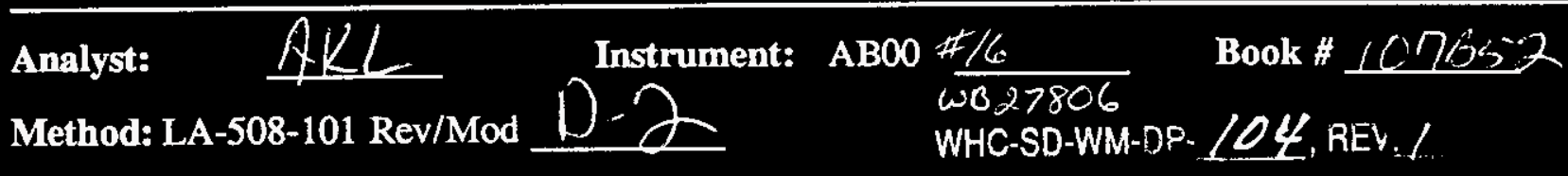

Worklist Comment: Do a boil down on $100 \mathrm{~mL}$ sample. Use $100 \mathrm{~mL}$ A-SPK. SLF Rerun $\# 1$ SLF 4.18 .95

\begin{tabular}{|c|c|c|c|c|c|c|c|c|c|c|}
\hline \multirow[t]{6}{*}{ GROUP } & PROJECT & S TYPE & SAMPLE\# & RA & HEST & MATRIX & ACTUAL & FOUND & DL & UNIT \\
\hline & & $1 \mathrm{sto}$ & & & ALLPHAO1 ALPHA01 & SOL ID & & & $\mathbf{H} / \mathbf{A}$ & $\mathrm{uCi} / \mathrm{g}$ \\
\hline & & 1 STD & & & ALLPHA01 ALPHAO1E & SOLID & & & N/A & X Ct. Error \\
\hline & & 2 BLNK-PREP & & & ALPHA01 ALPHA03 & SOLID & & & N/A & $\omega C \mathrm{i} / \mathrm{g}$ \\
\hline & & 2 BLNK-PREP & & & AALPHAO1 ALPHAO1E & SOL ID & & & N/A & x ct. Error \\
\hline & & 3 BLNK/BKG & .27 & & ALPHA01 ALPKA01 & SOLID & & & $N / A$ & $\mathrm{uCi} / \mathrm{g}$ \\
\hline 95000025 & $B Y-103$ & 4 SAMPLE & 595T000508 & $0 F$ & ALPHAO1 ALPHAOI & SOLID & N/A & & & $\mathrm{uCi} / \mathrm{g}$ \\
\hline 95000025 & BY-103 & 4 SAMPLE & S95T000508 & $0 F$ & AALPHA01 ALPHA01E & SOLID & $N / A$ & & & X Ct. Error \\
\hline 95000025 & BY-103 & 5 DUP & $595 \mathrm{r} 000508$ & $0 \mathrm{~F}$ & ALAPHA01 ALPHA01 & SOLID & & & N/A & $\mathrm{uCi} / \mathrm{g}$ \\
\hline 95000025 & $B Y-103$ & 5 DUP & 5957000508 & $0 \mathrm{~F}$ & ALPHAO1 ALPHA01E & SOLID & & & N/A & X Ct. Error \\
\hline 95000025 & BY -103 & 6 SPK & $595 T 000508$ & OF & DALPHA01 ALPHAO1 & SOLID & & & $N / A$ & uCi/g \\
\hline 95000025 & $B Y-103$ & 7 SAMPLE & 5957000512 & $O F$ & AALPHA01 ALPHA01 & SOL 10 & $\mathrm{~N} / \mathrm{A}$ & & & $\mathrm{uCi} / \mathrm{g}$ \\
\hline 95000025 & $B Y-103$ & 7 SAMPLE & $\$ 951000512$ & OF & ALPHAO1 ALPHA01E & SOL ID & $\mathbf{N} / \mathbf{A}$ & & & * Ct. Error \\
\hline 95000025 & $B Y-103$ & 8 DUP & $595 \mathrm{~T} 000512$ & O F & AALPHAOI ALPHAO1 & SOL ID & & & N/A & uCi/g \\
\hline 95000025 & $B Y-103$ & 8 DUP & 5957000512 & OF & AALPHAO1 ALPHAO1E & SOLID & & & N/A & X Ct. Error \\
\hline 95000025 & $8 Y-103$ & 9 SPK & $\$ 957000512$ & $0 F$ & AALPHA01 ALPHA01 & SOL 10 & & & $\mathrm{~N} / \mathrm{A}$ & $\mathrm{uCi} / \mathrm{g}$ \\
\hline
\end{tabular}

Final page for worklist \# 1148

CLenis 4/18195

Shavon Lllakgn $4-19.95$

Data Entry Comments:

SPK recoucry is below control linits-this is cousistent with the fiest ruw and ieddeates a suple matrix proble

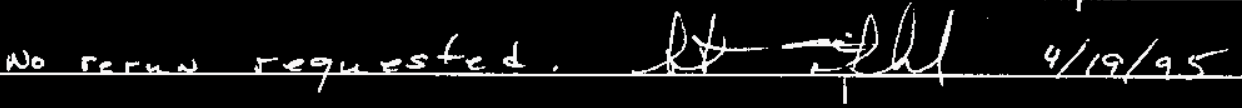

Units shown for $Q C$ (SPK \& STD) may not reflect the actual units. $D L=$ Detection Limit, $S=$ Worklist Slot Number, $R=$ Replicate Number, $A=$ Aliquot Code. 


\section{LABCORE Data Entry Template for Worklist\# 1148}

GROUP PROJECT

Analyst Signature

5 TYPE Date
SAMPLE\#

RA $\cdots \cdots \cdot$ TEST $\cdots \cdots$

MATRIX ACTUAL

Analyst Signature

FOUND

DL

UNIT

WHC-SD-WM-DP- /QL, REV. I.

Data Entry Comments:

Units shown for $Q C$ (SPK \& STD) may not reflect the actual units. $D L=$ Detection Limit, $S=$ Worklist Slot Number, $\boldsymbol{R}=$ Replicate Number, $\boldsymbol{A}=$ Aliquot Code. 
PLACE ANALYTICAL CARD IN BOX BELOW OR ATTACH TRAVELER

WHC-SD-WM-DP- 1 QC, REV

AT : LA-508-101 (D-2)

LA-548-101 (A-3) LIQUIDS

\begin{tabular}{|c|c|c|c|}
\hline ind & DETECTOR NUMBER & 16 & 16 \\
\hline SIANDARB & DISH SZE 1,2 , or 5 & 2 & 2 \\
\hline rerk & TOTAL COUNTS & 3749 & 3798 \\
\hline 146 & COUNT TIME in MINUTES & 30 & 30 \\
\hline 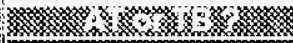 & BACKGROUND in cpm & 0.5 & 0.5 \\
\hline AT & SAMPLE SZZE in $\mathrm{mL}$ & 10.000 & 10.000 \\
\hline sos & DILUTION FACTOR & 1 & 1 \\
\hline AlPRPA & DIGEST DILUTION FACTOR & 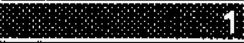 & 1 \\
\hline . & EFFICIENCY FACTOR & 0.2104 & 0.2104 \\
\hline 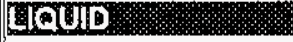 & Lc, Rmax, or Rs,(SAMPLE RATE) as APPROPRIATE & 124.467 & 126.100 \\
\hline$=0 ; 13$ & Sample Concentration in $\mu \mathrm{CI} / \mathrm{L}$ & $2.66 \mathrm{E}-02$ & BOOK\# \\
\hline WRKLST 1169 & Replicate Concentration in $\mu \mathrm{C} / \bar{L}$ & $2.70 E-02$ & $107 B 52$ \\
\hline
\end{tabular}

W

18327806.

Average Concentration in $\mathrm{HCill}$

2.6822E-02

AKL

Rs (Sample Count Rate) $=$ (TC/CT) - BKG

ALPHA TOTAL $\mu \mathrm{Ci} / \mathrm{L}=\mathrm{Rs} * 1000 \mathrm{~mL} / \mathrm{L}$ * DF * DDF / ( EFF * SS * $2220000 \mathrm{dpm} / \mu \mathrm{Ci}$ )

ALPHA TOTAL $\mu \mathrm{Ci} / \mathrm{mL}=$ ALPHA TOTAL $\mu \mathrm{Ci} / \mathrm{L} / 1000 \mathrm{~mL} /$

Relative Counting Error $=[\mid(T$ The Square Root of TC + BKG * CT) / (TC - BKG * CT) $]$ * $1.96 * 100$

Detection Levels and Less Than Values are determined from Procedure LA-508-002.

$\checkmark$ RESULTS v

\begin{tabular}{|lll|l|}
\hline ALPHA TOTAL in $\mu \mathrm{Ci} / \mathrm{mL}$ (Average) $=$ & $2.68 E-05$ & $\begin{array}{c}\text { DETECTION } \\
\text { LEVEL }\end{array}$ \\
\hline RELATIVE COUNTING ERROR & $=$ & & $1.48 \mathrm{E}-07$ \\
$\mu \mathrm{Ci} / \mathrm{mL}$ & $3.2 \%$ & \\
\hline
\end{tabular}

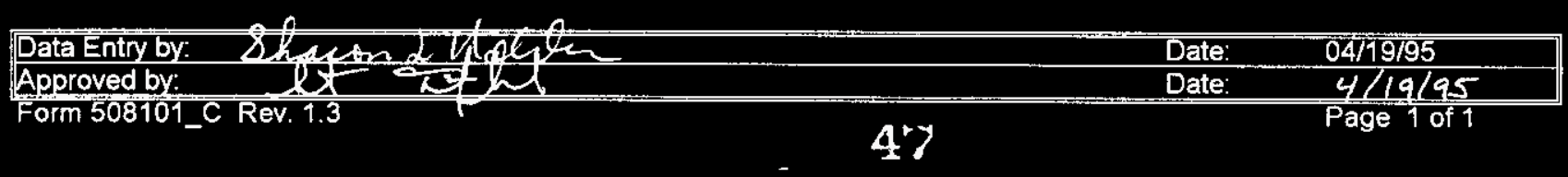


PLACE ANALYTICAL CARD IN BOX BELOW OR ATTACH TRAVELER

WHC-SD-WM-Dr 104 , REV 1

AT : LA-508-101 (D-2)

LA-548-101 (A-3) SOLIDS

\begin{tabular}{|c|c|c|c|}
\hline & & & 16 \\
\hline BL_ANR & DISH SIZE $\quad 1,2$, or 5 & 2 & 2 \\
\hline rmex & TOTAL COUNTS & 1 & 3 \\
\hline 1248 & COUNT TIME in MINUTES & 30 & 30 \\
\hline (x) & BACKGROUND in cpm & 0.5 & 0.5 \\
\hline AT & SAMPLE SIZE in $\mathrm{mL}$ & 0.100 & 0.100 \\
\hline $\sin (x)$ & DILUTION FACTOR & 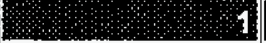 & 1 \\
\hline (OAXPLAOT & DIGEST GRAMS of SOLIDSIL & 2.0004 & 2.0004 \\
\hline mink & EFFICIENCY FACTOR & 0.2104 & 0.2104 \\
\hline 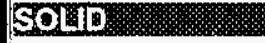 & Lc, Rmax, or Rs,(SAMPLE RATE) as APPROPRIATE & 0.301 & 0.301 \\
\hline $2 x_{3}$ & Sample Concentration in $\mu \mathrm{Ci} / \mathrm{g}$ & 3.22E-03 & BOOK\# \\
\hline S25TEDB. & Replicate Concentration in pCilg & $3.225-03$ & \\
\hline
\end{tabular}

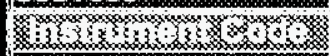

WE 77836

Maximum Concentration in $\mathrm{YCilg}$

$<\quad 3.2241 E-03$

(1)

AKL. $\quad R s$ (Sample Count Rate) $=(\mathrm{TC} / \mathrm{CT}$ ) $-\mathrm{BKG}$

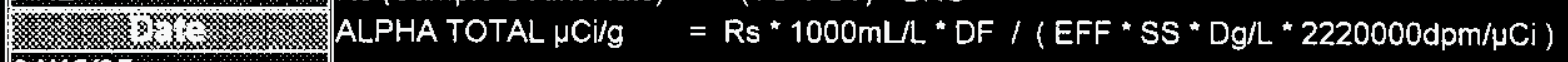

041895

xis

22.007)

Relative Counting Error $=[\mid(T h e$ Square Root of TC + BKG * CT) / (TC - BKG * CT) $]$ *1.96 * 100 Detection Levels and Less Than Values are determined from Procedure LA-508-002.

y RESULTS V

\begin{tabular}{|c|c|c|c|}
\hline ALPHA TOTAL & (Maximum) & $<3.22 E-03$ & \multirow{2}{*}{$\begin{array}{l}\text { DETECTION } \\
\text { LEVEL }\end{array}$} \\
\hline \multirow{2}{*}{\multicolumn{3}{|c|}{ LESS Than Value was Determined from Lc. }} & \\
\hline & & & \\
\hline RELATIVE COU & NG ERROR & $69.3 \%$ & uCilg \\
\hline
\end{tabular}

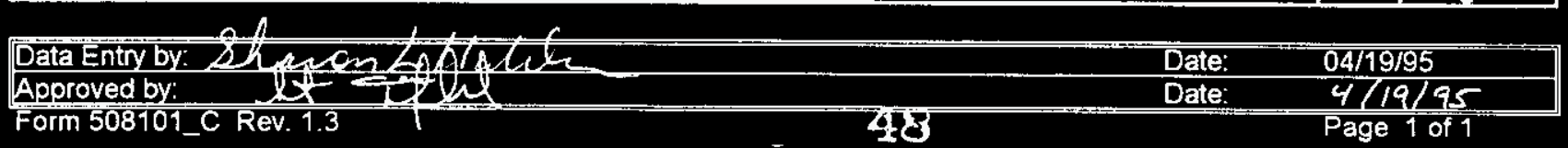


PLACE ANALYTICAL CARD IN BOX BELOW OR ATTACH TRAVELER WHC-SD-WM-D\%. 19.4 , REV.

\begin{tabular}{|c|c|c|c|}
\hline \multicolumn{4}{|c|}{ AT : LA-508-101 (D-2) } \\
\hline \multicolumn{2}{|c|}{ LA-548-101 (A-3) } & SAMPLE & REPLICATE \\
\hline 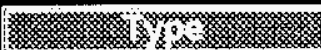 & DETECTOR NUMBER & 16 & 16 \\
\hline SAMPLE & DISH SIZE 1,2 , or 5 & (n) & 2 \\
\hline (1) & TOTAL COUNTS & 87 & 93. \\
\hline $1148 \times 1$. & COUNT TIME in MINUTES & m & 30 \\
\hline i & BACKGROUND in cpm & (1) & 0.5 \\
\hline AT & SAMPLE SIZE in $\mathrm{mL}$ & 0.100 & 0.100 \\
\hline 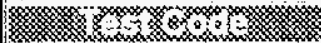 & DILUTION FACTOR & 1 & 1 \\
\hline OALPLAOT & DIGEST GRAMS of SOLIDSIL. & 2,0004 & 2.0004 \\
\hline . & EFFICIENCY FACTOR & 0.2104 & 0.2104 \\
\hline SOL10 & Lc, Rmax, or Rs,(SAMPLE RATE) as APPROPRIATE & 2.400 & 2.600 \\
\hline Whishos & Sample Concentration in $\mu \mathrm{Ci} / \mathrm{g}$ & 2.57E-02 & BOOK\# \\
\hline 5951508 & Replicate Concentration in $\mu \mathrm{Clig}$ & $2.78 E-02$ & \\
\hline (x) & & & \\
\hline WB27806 & Average Concentration in $\mu \mathrm{Clig}$ & $2.6756 E-02$ & \\
\hline is & & & \\
\hline AKL: & Rs (Sample Count Rate) & & \\
\hline istus & $=R s * 1000 \mathrm{~mL} / \mathrm{L} * \mathrm{DF} /(\mathrm{EFF} * \mathrm{~s}$ & * Dg/L *2220000d & $\mathrm{m} / \mu \mathrm{Ci})$ \\
\hline Q418195 & & & \\
\hline 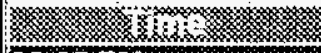 & Relative Counting Error $=[$ l $($ The Square Root of TC + BKC & " CT) / (TC - BKG & CT)| $] * 1.96 * 100$ \\
\hline 280012W & Detection L evels and L ese Than Values = & rocedure LA-508-00 & \\
\hline
\end{tabular}

V RESULTS V

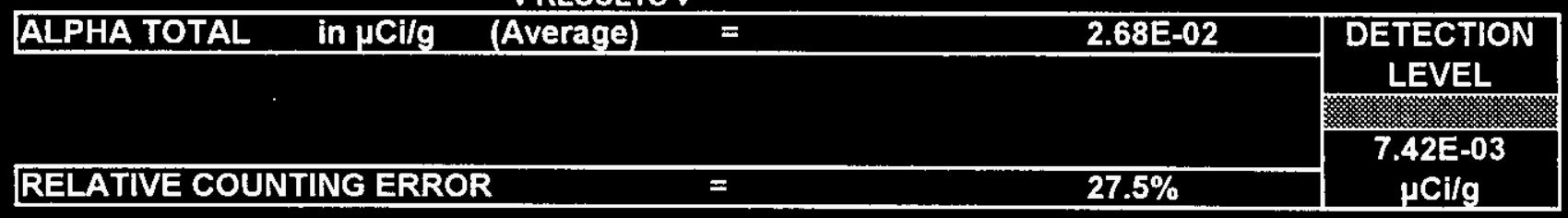

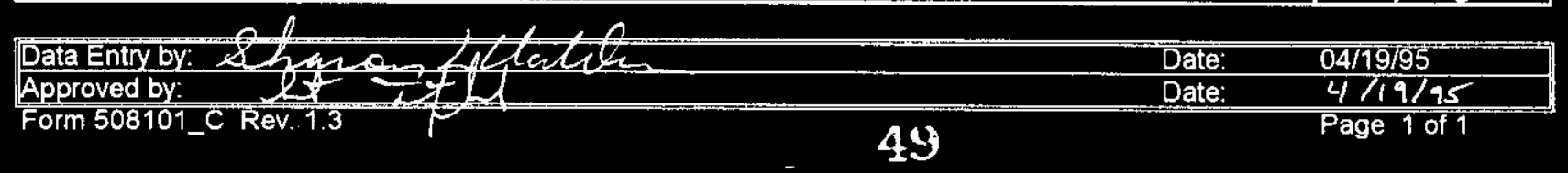


PLACE ANALYTICAL CARD IN BOX BELOW OR ATTACH TRAVELER WHC-SD-WM-DF. 104 , REV. $L$

\begin{tabular}{|c|c|c|c|}
\hline \multicolumn{4}{|c|}{ AT : LA-508-101 (D-2) } \\
\hline \multicolumn{2}{|c|}{ LA-548-101 (A-3) } & \multirow[t]{2}{*}{ SAMPLE } & REPLICATE \\
\hline 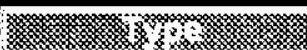 & DETECTOR NUMBER & & 16 \\
\hline 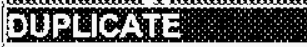 & DISH SIZE 1,2 , or 5 & 2 & 2 \\
\hline 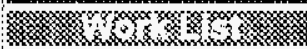 & TOTAL COUNTS & (18.8. & 61 \\
\hline 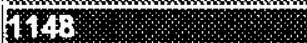 & COUNT TIME In MINUTES & 30 & 30 \\
\hline 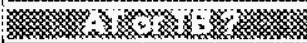 & BACKGROUND in cPm & 0.5 & 0.5 \\
\hline AT & SAMPLE SIZE in $\mathrm{mL}$ & 0.100 & 0.100 \\
\hline - & DILUTION FACTOR & 1 & 1. \\
\hline OALPRAM & DICEST GRAMS Of SOLIDSIL & 198 & 1.98 \\
\hline 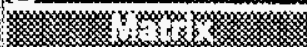 & EFFICIENCY FACTOR & 0.2104 & 0.2104 \\
\hline SOLID & Lc, Rmax, or Rs,(SAMPLE RATE) as APPROPRIATE & 2.200 & 1.533 \\
\hline 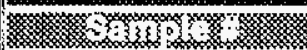 & Sample Concentration in $\mu \mathrm{Cilg}$ & $2.38 E-02$ & BOOK\# \\
\hline S957608 & Replicate Concentration in $\mathrm{pCl} / \mathrm{g}$ & $1.66 E-02$ & \\
\hline I. & & & \\
\hline WB227306 & Average Concentration in $\mathrm{PCl} / \mathrm{g}$ & $2.0184 E-02$ & \\
\hline$x_{3}$ & & & \\
\hline AKL & Rs (Sample Count Rate) & & \\
\hline alnglat & ALPHA TOTAL $\mu \mathrm{Ci} / \mathrm{g}=\mathrm{RS} * 1000 \mathrm{~mL} / \mathrm{L} * \mathrm{DF} /(\mathrm{EFF} * \mathrm{SS}$ & * Dg/L * 2220000 & $\mathrm{~m} / \mathrm{\mu Ci})$ \\
\hline r. & Relative Counting Error $=[1($ The Square Root of $\mathrm{TC}+\mathrm{BKG}$ & $\mathrm{CT}) /(\mathrm{TC}-\mathrm{Br}$ & \\
\hline $0220012 M$ & Detection Levels and Less Than Values are determined from & ocedure LA-508- & \\
\hline
\end{tabular}

V RESULTS v

\begin{tabular}{|lll|c|}
\hline ALPHA TOTAL in $\mu$ Cilg (Average) $=$ & $2.02 E-02$ & $\begin{array}{c}\text { DETECTION } \\
\text { LEVEL }\end{array}$ \\
\hline RELATIVE COUNTING ERROR & $=$ & & $\begin{array}{c}7.49 E-03 \\
\mu \mathrm{Ci} / \mathrm{g}\end{array}$ \\
\hline
\end{tabular}

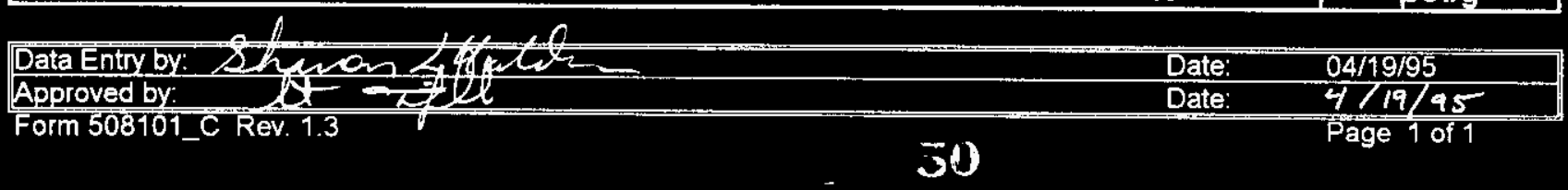




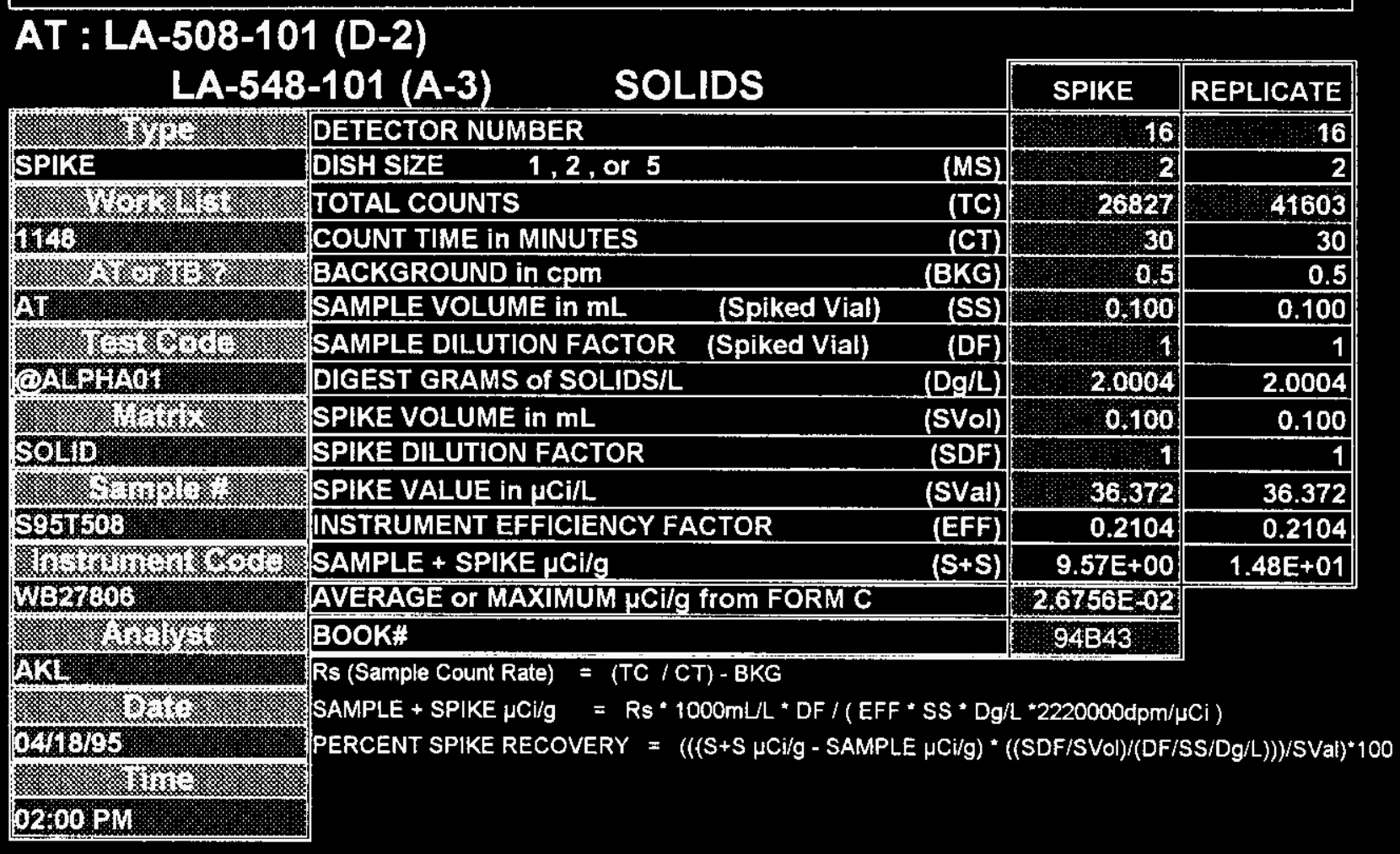

RESULT AVG. PERCENT SPIKE RECOVERY $=67.0 \%$

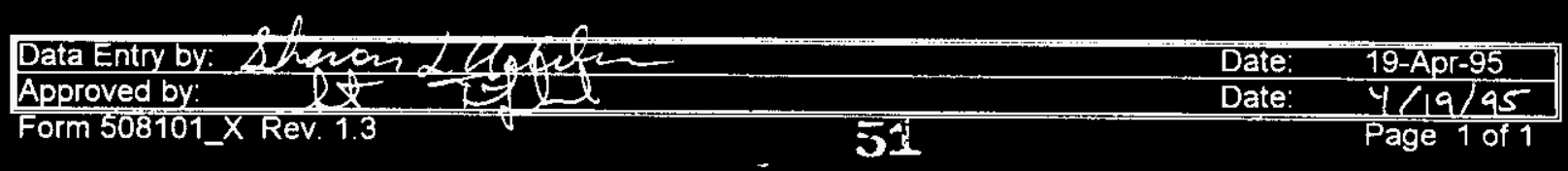




\section{AT : LA-508-101 (D-2)}

\section{LA-548-101 (A-3) SOLIDS}

\begin{tabular}{|c|c|}
\hline \\
\hline \multicolumn{2}{|l|}{ SAMPLE } \\
\hline \\
\hline \\
\hline 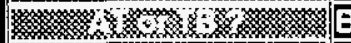 & \\
\hline at & \\
\hline - owo & \\
\hline \multicolumn{2}{|l|}{ 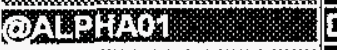 } \\
\hline sin & \\
\hline \multicolumn{2}{|l|}{ SOLID } \\
\hline 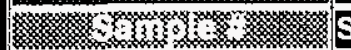 & \\
\hline \multicolumn{2}{|l|}{ S951512. } \\
\hline 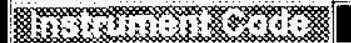 & \\
\hline \multicolumn{2}{|l|}{ WB27836 } \\
\hline N & \\
\hline \multicolumn{2}{|l|}{$A K L$} \\
\hline in & \\
\hline \multicolumn{2}{|l|}{2418395} \\
\hline Sisk & \\
\hline 22800200 & \\
\hline
\end{tabular}

DETECTOR NUMBER

DISH SIZE 1,2 , or 5

TOTAL COUNTS COUNT TIME in MINUTES

BACKGROUND in cpm

SAMPLE SLE in $\mathrm{mL}$

DILUTION FACTOR DIGEST GRAMS Of SOLIDSIL.

EFFICIENCY FACTOR

LC, Rmax, or Rs,(SAMPLE RATE) as APPROPRIATE

Sample Concentration in $\mu \mathrm{Cilg}$

Replicate Concentration in $\mu \mathrm{Ci} / \mathrm{g}$

\begin{tabular}{|c|c|c|}
\hline & SAMPLE & REPLICATE \\
\hline & 16 & 16 \\
\hline (MS) & 2 & 2 \\
\hline (TC) & (1) & 13 \\
\hline (CT) & 8 & 30 \\
\hline BKG) & 0.5 & 0.5 \\
\hline (SS) & 0.100 & 0.100 \\
\hline (DF) & 1 & 1 \\
\hline Dg $($ L) & 2.0164 & 2.0164 \\
\hline (EFF) & 0.2104 & 0.2104 \\
\hline & 0.378 & 0.301 \\
\hline$<$ & 4.01E-03 & BOOK\# \\
\hline $\bar{c}$ & $3.20 E-03$ & \\
\hline
\end{tabular}

Maximum Concentration in $\mu \mathrm{Cilg}$

$<$

4.0112E-03

Rs (Sample Count Rate) $=(T C / C T)-B K G$

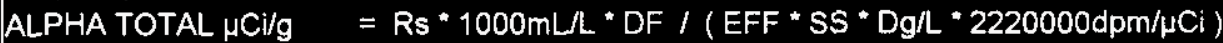

Relative Counting Error = [|(The Square Root of TC + BKG * CT) / (TC - BKG * CT) $]$ ] 1.96 * 100

Detection Levels and Less Than Values are determined from Procedure LA-508-002.

V RESULTS $v$

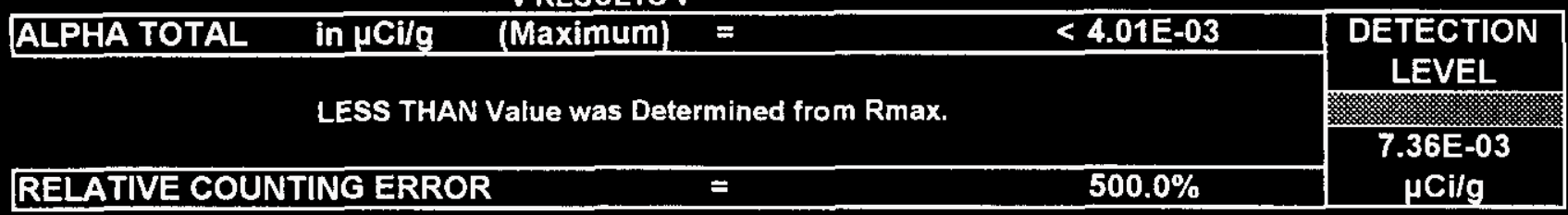

\begin{tabular}{l}
\hline Data Entry by: \\
Approved by:
\end{tabular}


PLACE ANALYTICAL CARD IN BOX BELOW OR ATTACH TRAVELER $\quad W H C-S D-W M-9 \mathrm{r}^{\circ} .104, \mathrm{nEV}$.

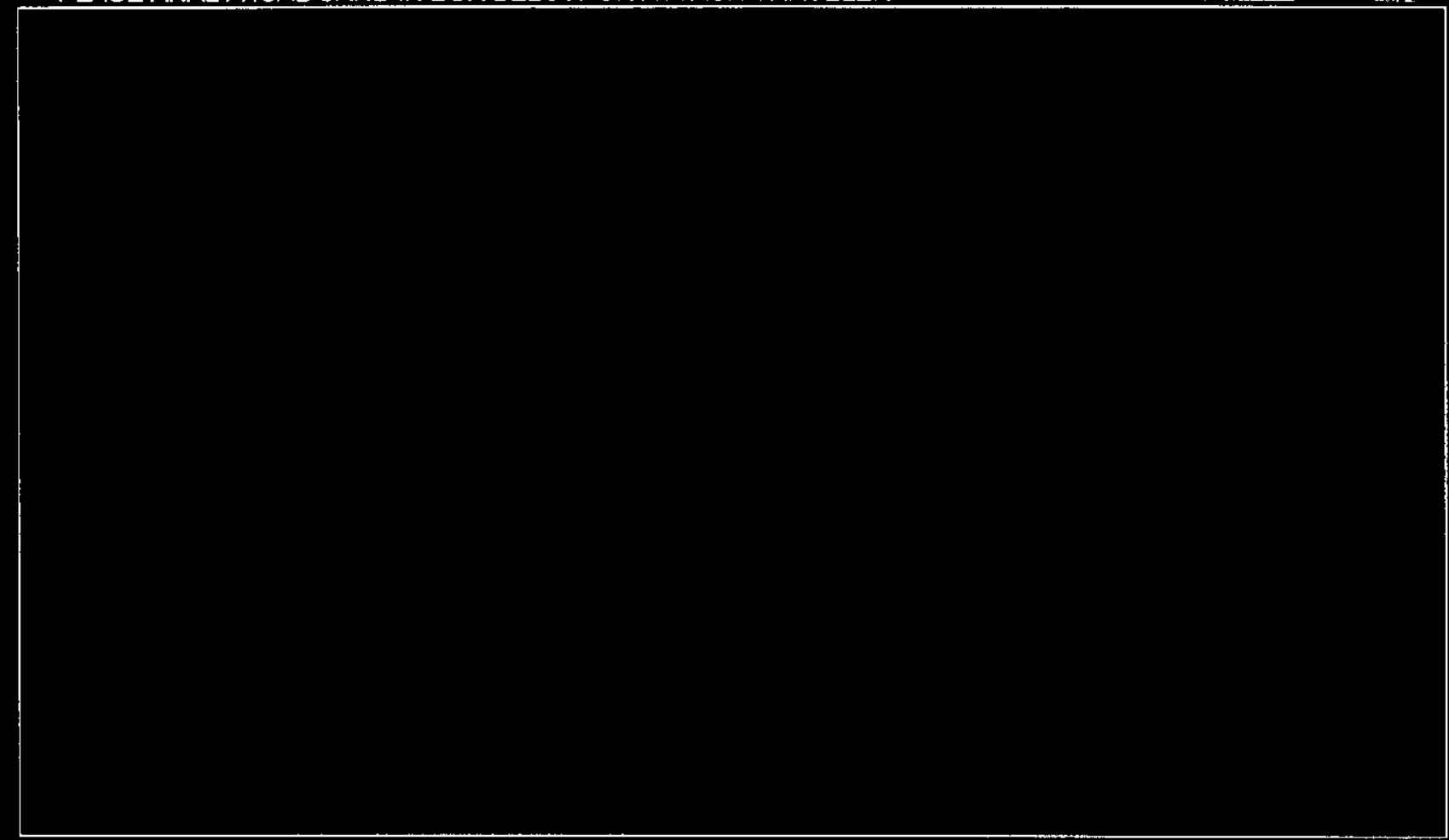

\begin{tabular}{|c|c|c|c|}
\hline \multicolumn{4}{|c|}{ AT : LA-508-101 (D-2) } \\
\hline \multicolumn{2}{|c|}{$L A-548-101(A-3)$} & \multirow[t]{2}{*}{ SAMPLE } & REPLICATE \\
\hline 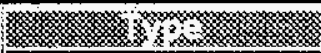 & DETECTOR NUMBER & & $\lcm{16}$ \\
\hline DUPLICATI & DISH SIZE $\quad 1,2$, or 5 & 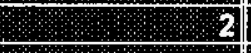 & 2 \\
\hline rom & TOTAL COUNTS & 14 & 5 \\
\hline 4146 & COUNT TIME in MINUTES & 30 & 30 \\
\hline 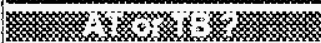 & BACKGROUND in cpm & 0.5 & 0.5 \\
\hline AT 2.28 & SAMPLE SIZE in $\mathrm{mL}$ & 0.100 & 0.100 \\
\hline m. & DILUTION FACTOR & m & 1 \\
\hline TALPHAO1 & DIGEST GRAMS of SOLIDSIL & 2.034 & 2.034 \\
\hline cotom & EFFICIENCY FACTOR & 0.2104 & 0.2104 \\
\hline SOU: & LG, Rmax, or Rs,(SAMPLE RATE) as APPROPRIATE & 0.301 & 0.301 \\
\hline *ustury & Sample Concentration in $\mu \mathrm{Ci} / \mathrm{g}$ & 3.17E-03 & BOOK\# \\
\hline S951512 & Replicate Concentration in $\mu \mathrm{Ci} / \mathrm{g}$ & 3.17E-03 & \\
\hline 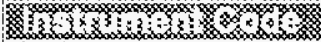 & & & \\
\hline ME27836 & Maximum Concentration in $\mu \mathrm{Ci} / \mathrm{g}$ & $3.1708 E-03$ & \\
\hline : & & & \\
\hline AKL & Rs (Sample Count Rate) & & \\
\hline a & $=R s * 1000 \mathrm{mLL} * \mathrm{DF} /(\mathrm{EFF} * \mathrm{~s}$ & ${ }^{*} \mathrm{Dg} / \mathrm{L} * 22200000$ & $\mathrm{~m} / \mu \mathrm{Ci})$ \\
\hline 0449895. & & & \\
\hline $02: 00 \mathrm{PH}$ & $\begin{array}{l}\text { Relative Counting Error = I / }(\text { The Square Root of TC + BKC } \\
\text { Detection Levels and Less Than Values are determined from } P\end{array}$ & $\left.{ }^{\circ} \mathrm{CT}\right) /(\mathrm{TC}-\mathrm{BKG}$ & CT) $\mid] * 1.96 * 10$ \\
\hline
\end{tabular}

v RESULTS $v$

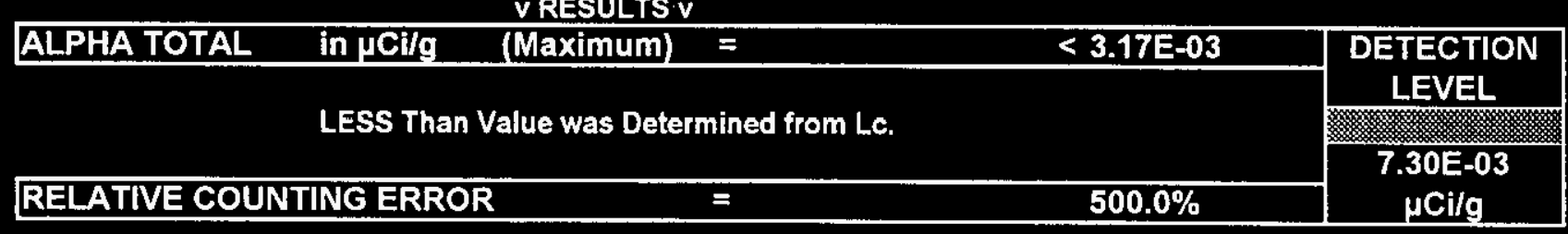

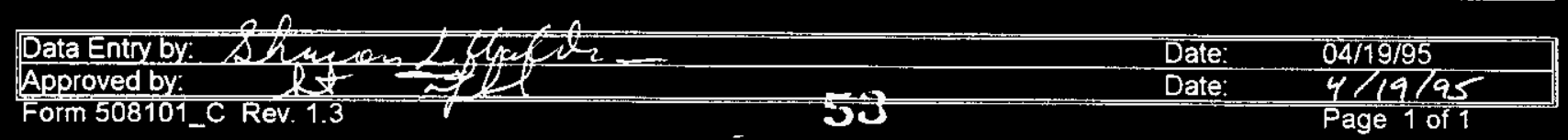


PLACE ANALYTICAL CARD IN BOX BELOW OR ATTACH TRAVELER WHC,SD-WM-DOF 104 , BFV. 1

\begin{tabular}{|c|c|c|c|}
\hline \multicolumn{2}{|c|}{ AT: LA-508-101 (D-2) } & \multirow[b]{2}{*}{ SPIKE } & \multirow[b]{2}{*}{ REPLICATE } \\
\hline LA-548 & SOLIDS & & \\
\hline 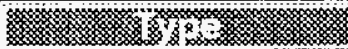 & DETECTOR NUMBER & 16 & 16 \\
\hline SPIKE & DISH SIZE $\quad 1,2$, or 5 & 2 & 2 \\
\hline mins & TOTAL COUNTS & 40604 & 36890 \\
\hline 1483 & COUNT TIME in MINUTES & 30 & 30 \\
\hline ind & BACKGROUND in cPm & 0.5 & 0.5 \\
\hline AT 2.0 & SAMPLE VOLUME in $\mathrm{mL}$ & 0.100 & 0.100 \\
\hline$x_{x}$ & SAMPLE DILUTION FACTOR (Spiked Vial) & 1 & 1 \\
\hline SALPHAB & DIGEST GRAMS of SOLIDS $/ L$ & 2.0164 & 2.0164 \\
\hline 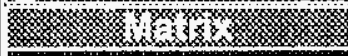 & SPIKE VOLUME in $\mathrm{mL}$ & 0.100 & 0.100 \\
\hline SQLID & SPIKE DILUTION FACTOR & -1 & 1 \\
\hline 20 & SPIKE VALUE in $\mu \mathrm{CI} / \mathrm{L}$ & 36.372 & 36.372 \\
\hline S951512 & NSTRUMENT EFFICIENCY FACTOR & 0.2104 & 0.2104 \\
\hline 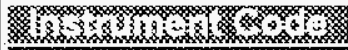 & SAMPLE + SPIKE $\mu \mathrm{Ci} / \mathrm{g}$ & $1.44 E+01$ & $1.31 \mathrm{E}+01$ \\
\hline Wa27806 & AVERAGE or MAXIMUM $\mu \mathrm{CI} / \mathrm{g}$ from FORM C & K40112E 3 & \\
\hline W & BOOK\# & 94843 & \\
\hline Ars & Rs (Sample Count Rate) $=(\mathrm{TC} / \mathrm{CT})-\mathrm{BKG}$ & & \\
\hline$-x_{3}$ & SAMPLE + SPIKE $\mu \mathrm{Ci} / \mathrm{g}=\mathrm{RS} * 1000 \mathrm{mLL} \cdot \mathrm{DF} /(\mathrm{EFF} \cdot \mathrm{SS} * \mathrm{Dg} /$ & $L \cdot 2220000 \mathrm{dpm} / \mathrm{f}$ & \\
\hline 0.410196 & PERCENT SPIKE RECOVERY $=\left(\left((S+S \mu C l / g-S A M P L E ~ \mu C i / g){ }^{*}\right.\right.$ & $((\mathrm{SDF} / \mathrm{SVO}) /(\mathrm{DF} / \mathrm{S}$ & SS/Dg/L)/)/SVal)*1 \\
\hline 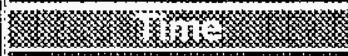 & & & \\
\hline $32100 \mathrm{PH}$ & & & \\
\hline
\end{tabular}

RESULT AVG. PERCENT SPIKE RECOVERY $=76.0 \%$

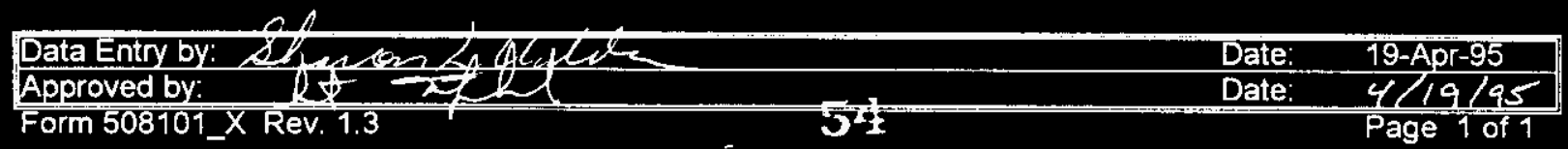




\section{(w) Westinghouse \\ Hanford Company}

P.O. Box 1970 Richland. WA 99352

PART II

WHC-SD-WM-DP. 104, REV. I

WHC-SD-WM-DP-104, REV. 0

ANALYTICAL SERVICES 95-AUG-012 AND 95-AUG-013

DATE PRINTED:

APRIL 20, 1995 
WHC-SD-WM-DP-104, REV. 0

\section{TABLE OF CONTENTS}

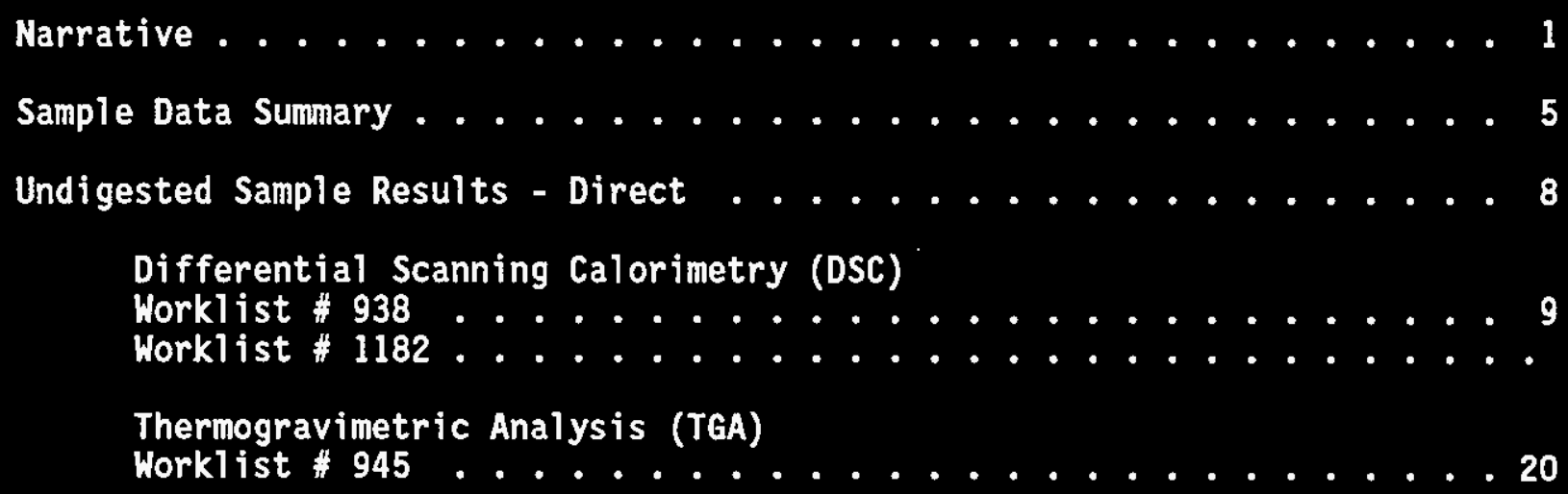

This Document consists of pages 1 through 28. 
WHC-SD-WM-DP-104, REV. 1

NARRATIVE

53 
THIS PAGE INTENTIONALWY LEFT BLANK 


\author{
WHC-SO-WM-OP-104, RE: I \\ WHC-SD-WM-DP-104, REV. 0 \\ 45-DAY SAFETY SCREEN RESULTS FOR TANK 241-8Y-103, \\ AUGER SAMPLES 95-AUG-012 AND 95-AUG-013
}

\title{
ANALYTICAL SUMMARY
}

Two auger samples from tank 241-BY-103 (BY-103) were received by the 222-S Laboratories and underwent safety screening analysis, consisting of

differential scanning calorimetry (DSC), thermogravimetric analys is (TGA), and total alpha activity. Analytical results for the TGA analyses for both samples were less than the safety screening notification limit. Since notification is made if the sample is analyzed at less than 17 percent water, notification was made on April 20, 1995. Although the sample results were below this limit, no secondary analyses were required or performed.

\section{SCOPE}

This document serves as the 45-day report deliverable for the tank BY-103 auger samples collected on March 8, 1995 and March 10, 1995 (95-AUG-012 and 95-AUG-013). Each sample was received, extruded, and analyzed by the 222-S Laboratories in accordance with the below referenced TCP. Included in this report are the primary safety screening results obtained from the analyses and copies of all DSC and TGA raw data SCans as requested per the TCP.

Photographs of the auger samples were taken during extrusion and, although not included in this report, are available.

\section{SAMPLE RECEIPT, EXTRUSION; AND SUBSAMPLING}

\section{Auger 95-AUG-012}

Auger sample 95-AUG-012 was collected from riser $10 \mathrm{~B}$ of tank BY-103 using a 10 inch auger bit. It was received into the 222-S Laboratories on March 10, 1995 at 1600 hours and extruded on March 23, 1995. There was no drainable liquid, but 35.85 grams of solids were collected. The solids were brownish-grey in color, looked dry and crumbiy, and fell off the auger onto the sample tray after the sleeve was removed. Due to the small amount of sample obtained, all material was subsampled into one jar ( $\# 6570$ ) and was not divided into half-segments as directed in the TCP. All analys is and sample archiving requirements per the TCP were performed on this sample.

Auger 95-AUG-013

Auger sample 95-AUG-013 was collected from riser 12A of tank BY-103 using a 10 inch auger bit. It was received into the 222-S Laboratories on March 10, 1995 at 1600 hours and extruded on March 24, 1995. There was no drainable liquid, but $38.09 \mathrm{grams}$ of solids were collected. The solids were yellow-brown in color, looked dry and crumbly, and fell off the auger onto the sample tray after the sleeve was removed. As with the sample material from auger 95-AUG-012, the material from 95-AUG-013 was subsampled into one jar (\#6578) from which all analyses and sample archives were performed. 


\author{
WHC-SD-WM-DQ. 104 , RFV / \\ WHC-SO-WM-DP-104, REV. 0
}

SAMPLE IDENTIFICATION INFORMATION

Table 1: Sample Identification

\begin{tabular}{|c|c|c|c||}
\hline $\begin{array}{c}\text { LABCORE } \\
\text { Sample ID }\end{array}$ & Customer Sample ID & $\begin{array}{c}\text { Hot Cell } \\
\text { Vial \# }\end{array}$ & Analyses \\
\hline \hline S95T000320 & $95-A U G-012$, Riser 10B, Auger sample & NA & Extrusion \\
\hline S95T000507 & $95-A U G-012$, Riser 10B, Subsample & 6668 & DSC/TGA \\
\hline S95T000508 & $95-A U G-012$, Riser 10B, Fusion Digest & 6668 & Total Alpha \\
\hline S95T000509 & $95-A U G-012$, Riser 10B, Archive Sample & 6756 & Archive \\
\hline S95T000321 & $95-A U G-013$, Riser 12A, Auger Sample & NA & Extrusion \\
\hline S95T000511 & $95-A U G-013$, Riser 12A, Subsample & 6810 & DSC/TGA \\
\hline S95T000512 & $95-A U G-013$, Riser 12A, Fusion Digest & 6810 & Total Alpha \\
\hline S95T000513 & $95-A U G-013$, Riser 12A, Archive Sample & 6877 & Archive \\
\hline
\end{tabular}

NA $=$ Not Applicable

\title{
ANALYTICAL RESULTS
}

Analytical results are presented in Tables 2 and 3, with the applicable notification 7 imits shaded.

\section{DSC (Energetics Content)}

DSC analyses were performed under a nitrogen atmosphere using procedure LA-514-113, Rev. B-1. Using the average percent water by TGA for each sample, dry weight basis results were calculated from the wet weight basis results. Both the wet and dry results are given in Tables 2 and 3 . For both sample S95T000507 and sample S95T000511, exotherms ranging from 5.9 to $137.6 \mathrm{~J} / \mathrm{g}$ (dry weight basis) were detected beginning at approximately $400{ }^{\circ} \mathrm{C}$; however, the magnitude of these exotherms did not approach the notification 1 imit of $481 \mathrm{~J} / \mathrm{g}$. Due to the inhomogeneity of the samples, neither sample met the TCP specified precision criterion of $10 \%$ relative percent difference (RPD). Sample precision values were 19.2 and 59.5 RPD for samples S95T000507 and 5957000511, respectively. The LMCS control standard exhibited a recovery of 104.7 percent, which was within the program's specified accuracy control 1 imits of 90 to 110 percent recovery. Since the sample precision values did not meet the specified precision identified in the TCP, an additional analysis was performed for each of the two samples. The results for these additional analyses were 77.1 and $11.3 \mathrm{~J} / \mathrm{g}$ (calculated to be 94.9 and $13.4 \mathrm{~J} / \mathrm{g}$ on a dry weight basis) for samples S95T000507 and S95T000511, respectively. These results do not appear in Tables 2 and 3 but are included in the raw data. 
WHC-SD-WM-DP- 10.4, REV - 1

WHC-SD-WM-DP-104, REV. 0

\section{IGA (Moisture Content)}

Weight percent water was performed under a nitrogen atmosphere using procedure LA-560-112, Rev. A-2. Results for samples S95T000507 and S95T000511 and their duplicates ranged in value from 11.63 to 33.59 percent water by weight. The marked difference in the results is most 7 ikely attributable to the inhomogeneity of the sample material. The results for both samples were less than the notification 1 imit of 17 weight percent water, al though the results of the duplicates samples were greater than this limit (notification is made if the sample is analyzed at less than 17 percent water). The RPD values between the samples and their duplicates were 82.0 and 50.5 for samples $\$ 95 T 000507$ and $595 T 000511$, respectively, and did not meet the TCP specified precision requirement of $10 \%$. The Laboratory Measurement Control System (LMCS) control standard exhibited a recovery of 99.98 percent, which was within the program's specified accuracy control limits of 90 to 110 percent. Since the sample precision values did not meet the specified precision identified in the TCP, an additional analysis was performed for each of the two samples. The results for these additional analyses were 18.76 and 15.72 percent water by weight for samples S95T000507 and S95T000511, respectively. These results do not appear in Tables 2 and 3 but are included in the raw data.

\section{Total Aloha Activity}

Although not required by the TCP for saltcake samples, analyses for total alpha activity were performed on samples S95T000508 and S95T000512. Samples were prepared by fusion using procedure LA-549-141, Rev. C-2, and analyses were performed using procedure LA-508-101, Rev. D-2. A sample duplicate and spike were performed on both samples. Neither sample met the TCP specified precision criterion of $10 \%$ RPD. Likewise, lower than normal spike recoveries caused both samples to exceed the accuracy control 7 imits of 90 to 110 percent recovery, with values of $67.0 \%$ and $76.0 \%$. This poor spike recovery was most likely due to solids observed in the sample mount. An additional run was performed for both samples from which similar results were found. The results of the rerun are given in Tables 2 and 3 . Sample and duplicate alpha activities ranged from less than $3.17 \mathrm{E}-03$ to $2.68 \mathrm{E}-02 \mu \mathrm{Ci} / \mathrm{g}$, which were more than 1500 fold lower than the notification limit of $41 \mu \mathrm{Ci} / \mathrm{g}$.

REFERENCE Schrejber, R. D., 1995, WHC-SD-WM-TP-231, Revision 1, "Tank 241-BY-103 Tank Characterization Plan, dated February 27, 1995. 


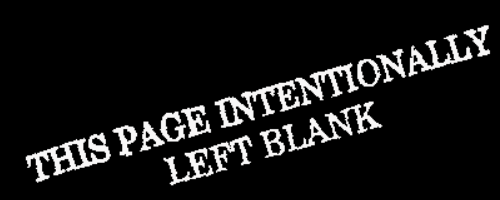


WHC-SD-WH-DP-104, REV. I

SAMPLE DATA SUMMARY

61 
THIS PAGE INTENTIONALLY

LEFT BLANK 


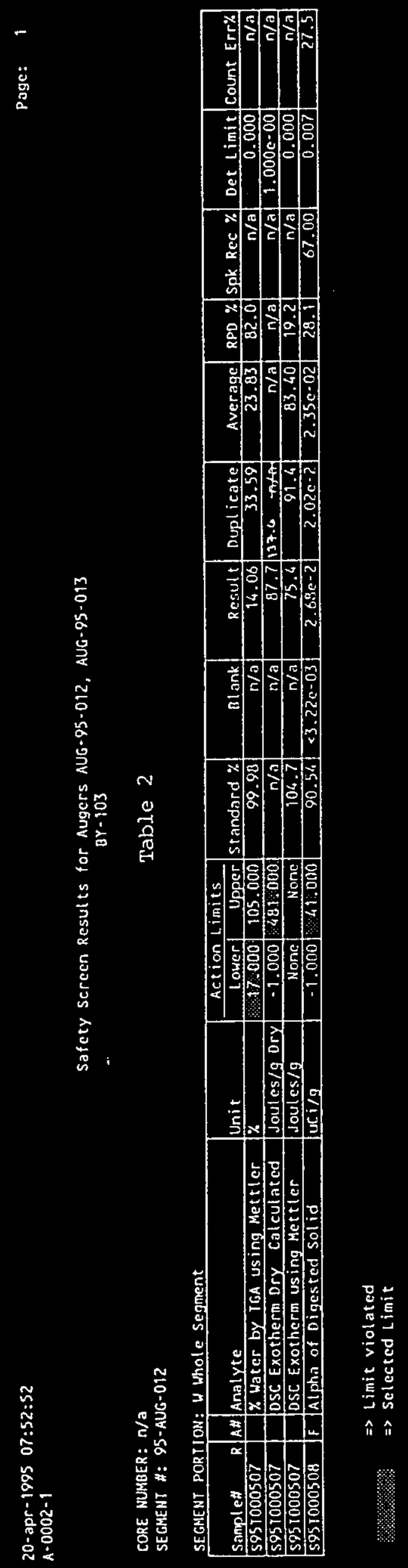


Safety Screen Results for Augers AUG-95-012, AUG-95-013 BY- 103

CORE HUMBER: $n / a$

SEGMENT \#: 95 -AUG.013

Table 3

SEGMENT PORTION: $W$ Whole Scgment

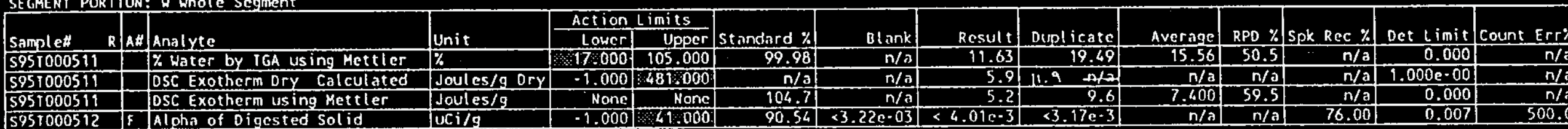

$\Rightarrow$ Limit violated

$\Rightarrow$ Selected Limit 
WHC-SD-WM-DP-104, REV . 1

UNDIGESTED SAMPLE RESULTS -DIRECT 
TIIS PAGE INTIENTIO NAIIIT
IEFT BLANK 


\section{LABCORE Data Entry Template for Worklist\# 938}

\begin{tabular}{|c|c|c|c|c|c|}
\hline Analyst: & $K R M$ & Instrument: & DSC01 & & Book \# 12N14A \\
\hline Method: & 4-113 Rev/ & $B-1$ & & WHC-SD-H & $\mathrm{APP}-104, \mathrm{REV} .1$ \\
\hline
\end{tabular}

Worklist Comment: Please run BY-103 DSC under N2. bdv

\begin{tabular}{|c|c|c|c|c|c|c|c|c|c|c|}
\hline \multirow[t]{2}{*}{ GROUP } & PROJECT & S TYPE & SAMPLE\# & R A & $\ldots$ - & MATRIX & ACTUAL & FOUND & $\mathrm{DL}$ & UNIT \\
\hline & & 1 STD & & & OSC+01 & SOL 10 & 25,45 & 24. & $N / A$ & Joules/g \\
\hline 95000025 & BY -103 & 2 SAMPLE & \$95T000507 & 0 & OSC-01 & SOL ID & $N / A$ & & & Joules/g \\
\hline 95000025 & $8 Y-103$ & 3 DUP & 5951000507 & 0 & DSC-01 & $50 L 10$ & & & $N / A$ & Joules/g \\
\hline 95000025 & $8 Y-103$ & 4 DUP2 & 5957000507 & 0 & $05 C-01$ & SOLID & & 77 & $\mathrm{~N} / \mathrm{A}$ & Joules/9 \\
\hline 85000025 & BY-103 & 5 SAMPLE & 5955000511 & 0 & DSC-01 & SOLID & $N / A$ & 5. & 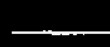 & Joules/g \\
\hline 95000025 & $B Y-103$ & 6 DUP & 5951000511 & 0 & DSC-01 & SOLID & & .6 & $N / A$ & Joules $/ g$ \\
\hline & & 7 STO & & & DSC-01 & SOL I & & & $N / A$ & Joules/g \\
\hline 95000025 & $B Y-103$ & 8 DUPZ & 5957000511 & 0 & OSC-01 & SOLID & 52 & 11.3 & $N / A$ & Joules $/ 9$ \\
\hline
\end{tabular}

\section{Final page for worklist \# 938}

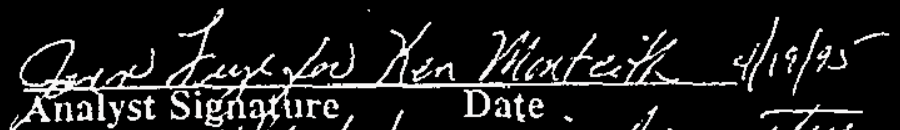

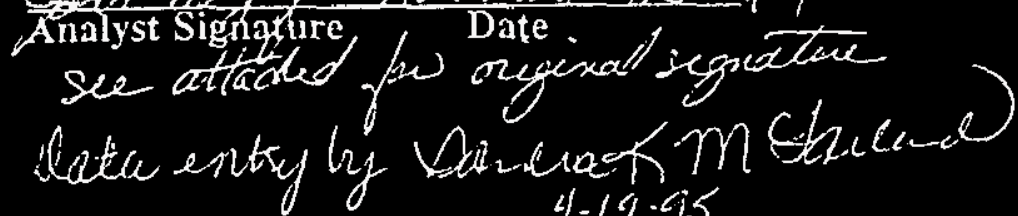

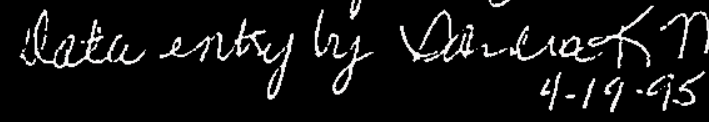
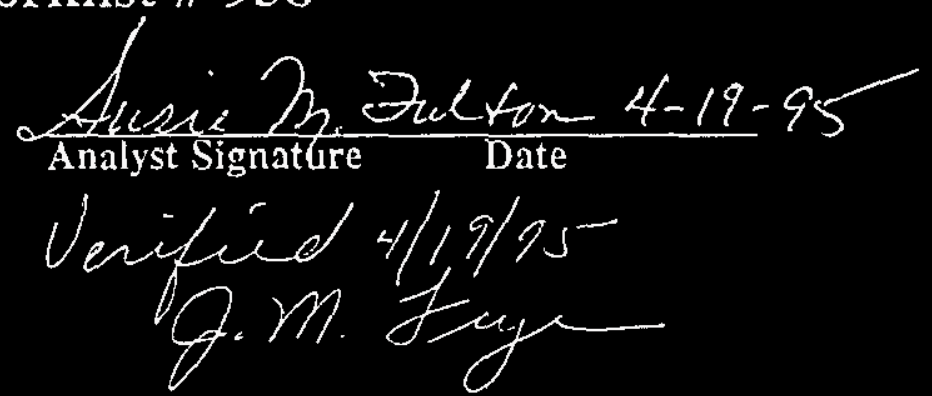

3957000507 has two endo themes $736.8 \mathrm{glg}$ at $125.4^{\circ} \mathrm{C}, 120.991 \mathrm{~g}$ at $302.7 \mathrm{C}$; tuplecate heo $609.79 \mathrm{lg}$ at $125 \mathrm{C}, 181.0 \mathrm{~g} / \mathrm{gg}$ at $300 \mathrm{c}$; dup2 has $508.8 \mathrm{glg}$ at $113^{\circ} \mathrm{C}, 258.5 \mathrm{glg}$ af $274^{\circ} \mathrm{C}$.

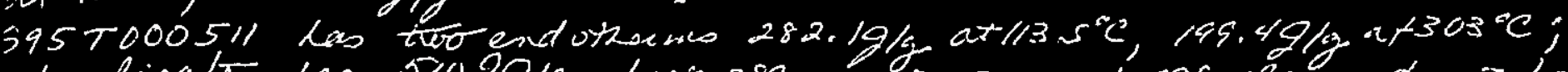
duplicate las $570.92 \mathrm{~g}$ at $108.2^{\circ} \mathrm{C}, 232.59 \mathrm{~g}$ at $298.4^{\circ} \mathrm{C} ;$ dup 2 hao $564.1 \mathrm{glg}$ at $108^{\circ} \mathrm{C}, 189.9 \mathrm{glg}$ at $296 \mathrm{C}$. Jom fuy allig/s5

Data Entry Comments: Buoum

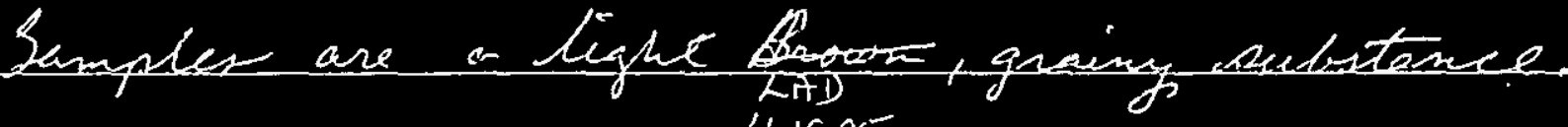
4. $-19-95$

Units shown for $Q C$ (SPK \& STD) may not reflect the actual units. $D L=$ Detection Limit, $S=$ Worklist Slot Number,

$R=$ Replicate Number, $A=$ Aliquot Code.

Gi 


\section{LABCORE Data Entry Template for Worklist\# 938}

Analyst: $\quad K R M$ Instrument: DSCO1

Method: LA-514-113 Rev/Mod $\beta-1$

Book \#12N14A

WHC-SD-WM-DP. LI4,REV.LL

Worklist Comment: Please run BY-103 DSC under N2. bdv

\begin{tabular}{|c|c|c|c|c|c|c|c|c|c|c|}
\hline \multirow[t]{2}{*}{ GROUP } & PROJECT & S TYPE & SAMPLE\# & R A & 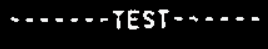 & MATRIX & ACTUAL & FOLND & $D L$ & UNIT \\
\hline & & 1 STD & & & DSC. 01 & SOLIO & & & N/A & Joules/g \\
\hline 95000025 & BY -103 & 2 SAMPLE & S95T000507 & 0 & OSC-01 & SOLID & $N / A$ & & & Joules $/ 9$ \\
\hline 95000025 & $B Y+103$ & 3 DUP & \$95T000507 & 0 & DSC-01 & SOLIO & & & N/A & Joules/g \\
\hline 95000025 & BY -103 & 4 SAMPLE & S95ro00511 & 0 & OSC-01 & SOLIO & $N / A$ & & & Joules $/ g$ \\
\hline 95000025 & $8 Y-103$ & 5 OUP & S95T000511 & 0 & DSC-01 & SOL 10 & & & N/A & Joules $/ \mathrm{g}$ \\
\hline
\end{tabular}

Final page for worklist \# 938

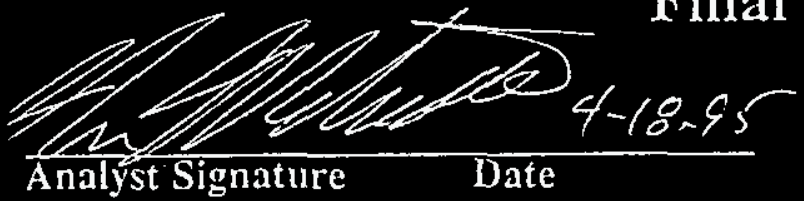

Analyst Signature Date

Data Entry Comments:

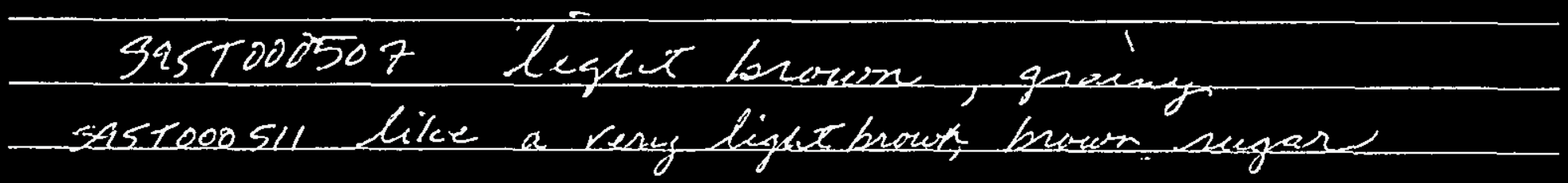

Units shown for $Q C$ (SPK \& STD) may not reflect the actual units. $D L=$ Detection Limit, $S=$ Worklist Slot Number,

$R=$ Replicate Number, $A=$ Aliquot Code.

Ge 


\section{LABCORE Data Entry Template for Worklist\# 1182}

Analyst: $\operatorname{TMF}$ Instrument: $\mathrm{DSC} 01 \ldots$ Book $\#$ N/A

Method: LA-514-113 Rev/Mod B-/

WHC-SD-WM-DP. 104, REV 1

Worklist Comment: Dry dsc BY-103. JMF

\begin{tabular}{|c|c|c|c|c|c|c|c|c|c|c|}
\hline GROUP & PROJECT & $S$ TYPE & SAMPLE\# & $R A$ & - TEST $\ldots . . .$. & MATRIX & ACTUAL & FOUND & $D L$ & UNIT \\
\hline 95000025 & $B Y-103$ & 1 SAMPLE & $595 T 000507$ & 0 & DSC -02 & SOLID & N/A & 87.7 & & Joules/g Dry \\
\hline 95000025 & $B Y-103$ & 2 DUP & $\$ 951000507$ & 0 & osc- 02 & SOL10 & 87.7 & 137.6 & N/A & Joules $/ 9$ Dry \\
\hline 95000025 & $8 Y+103$ & 3 DUP2 & S95T000507 & 0 & DSC-02 & SOL 10 & 87.7 & 94.9 & $\mathrm{~N} / \mathrm{A}$ & Joules $/ 9$ Dry \\
\hline 95000025 & $B Y-103$ & 4 SAMPLE & 5957000511 & 0 & DSC-02 & SOLID & $N / A$ & 5.9 & & Joules/g Dry \\
\hline 95000025 & $8 Y \cdot 103$ & 5 OUP & 5951000511 & 0 & DSC-02 & SOLIO & 5.9 & 11.9 & $N / A$ & Joules/g Ory \\
\hline 95000025 & $8 Y-103$ & 6 DUP2 & S95T000511 & 0 & DSC-02 & SOL 10 & 5.9 & $1+\infty$ & N/A & Joules/g Dry \\
\hline
\end{tabular}

Final page for worklist \# 1182

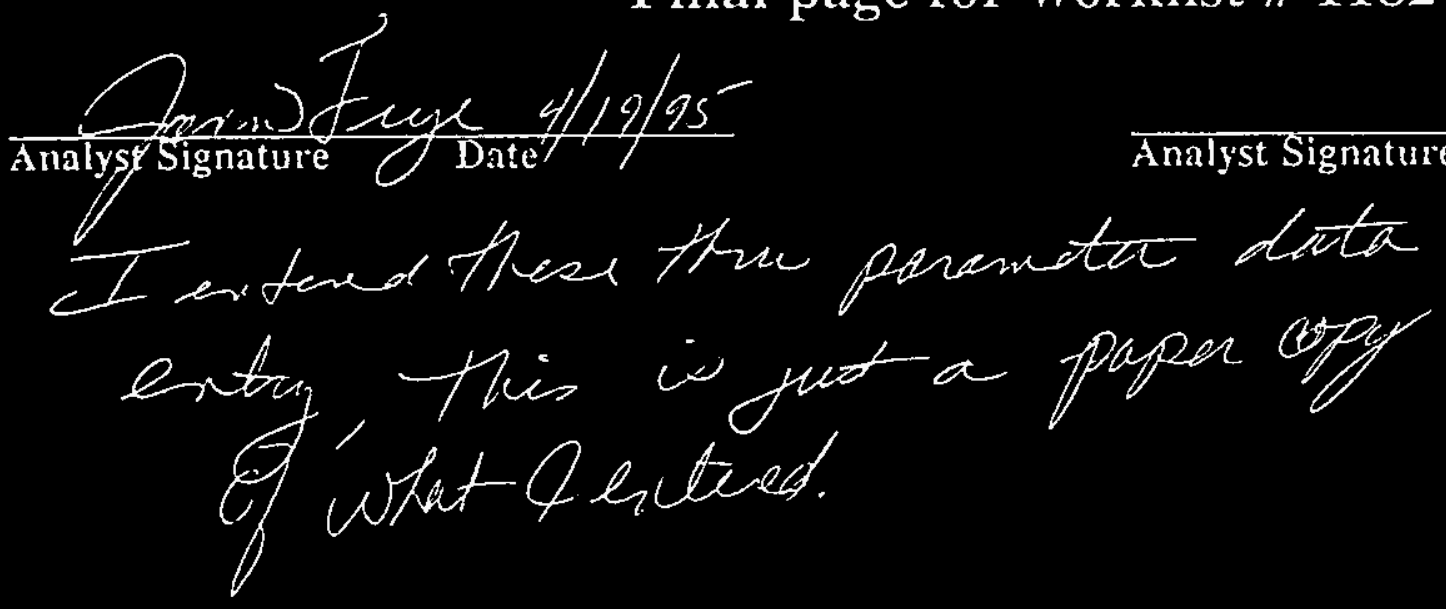

Data Enrry Comments:

Units shown for QC (SPK \& STD) moy not reflect the actual units. $D L=$ Detection Limit, $S=$ Worklist Slot Number; $R=$ Replicate Number, $A=$ Aliguot Code. 


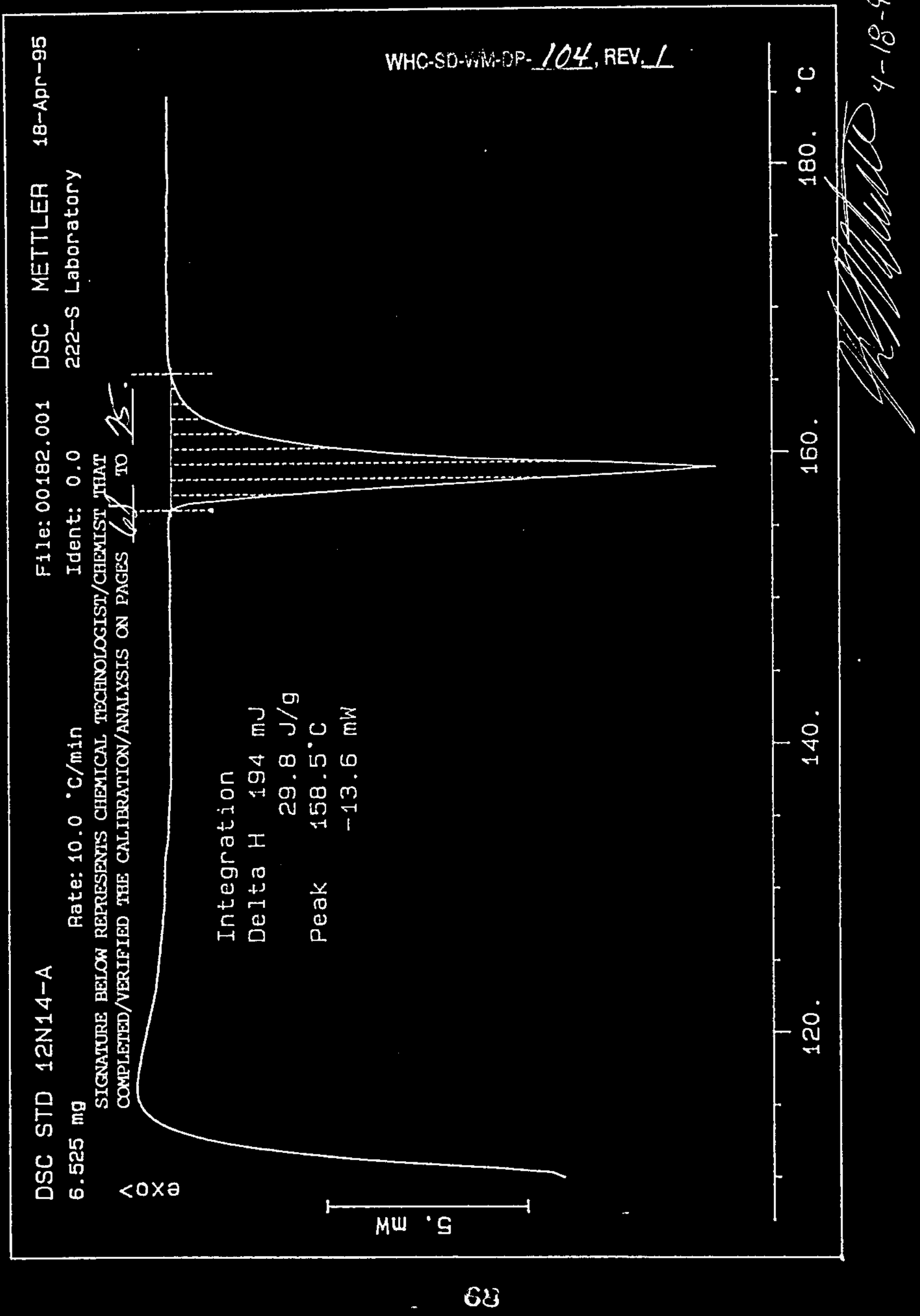




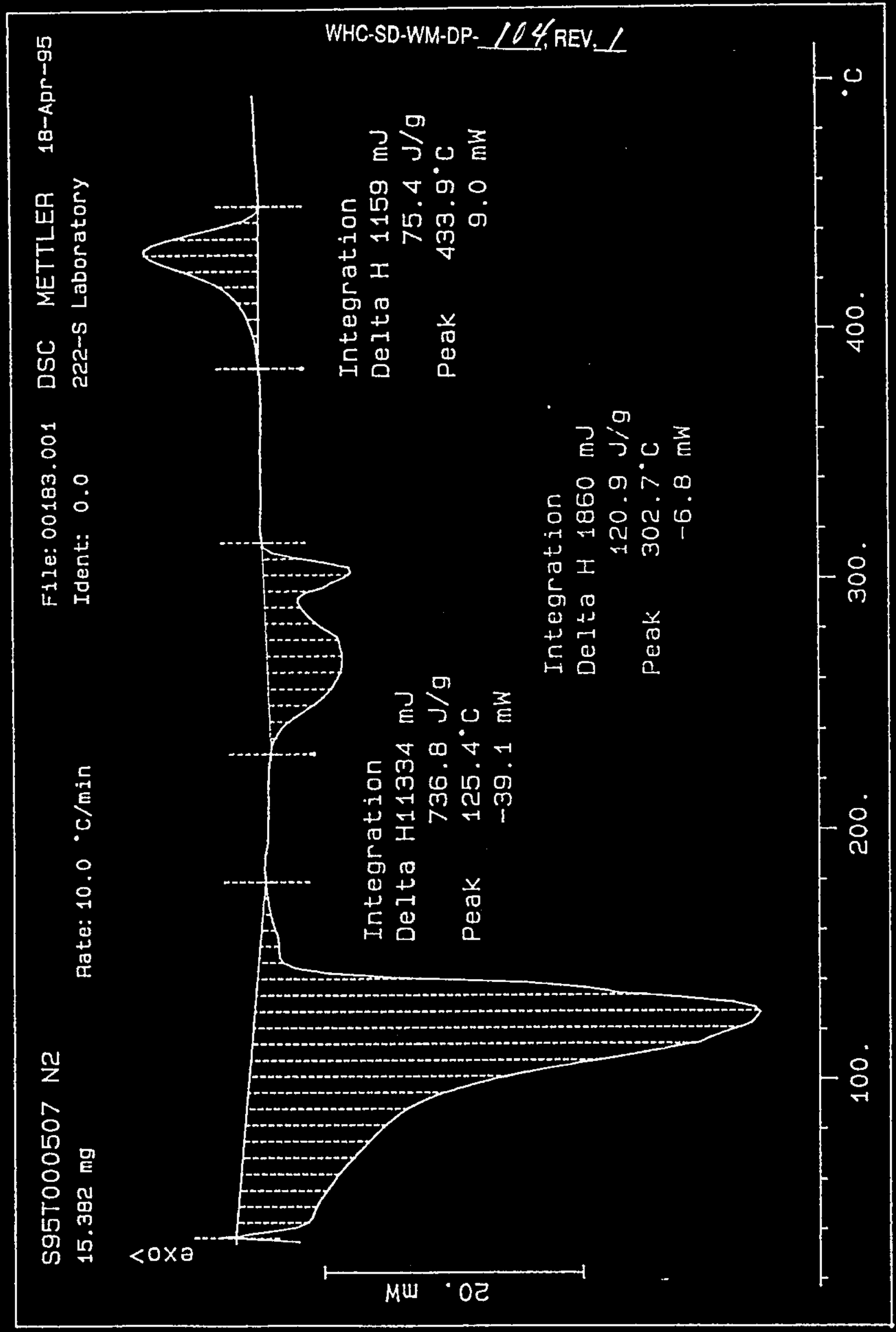

69 


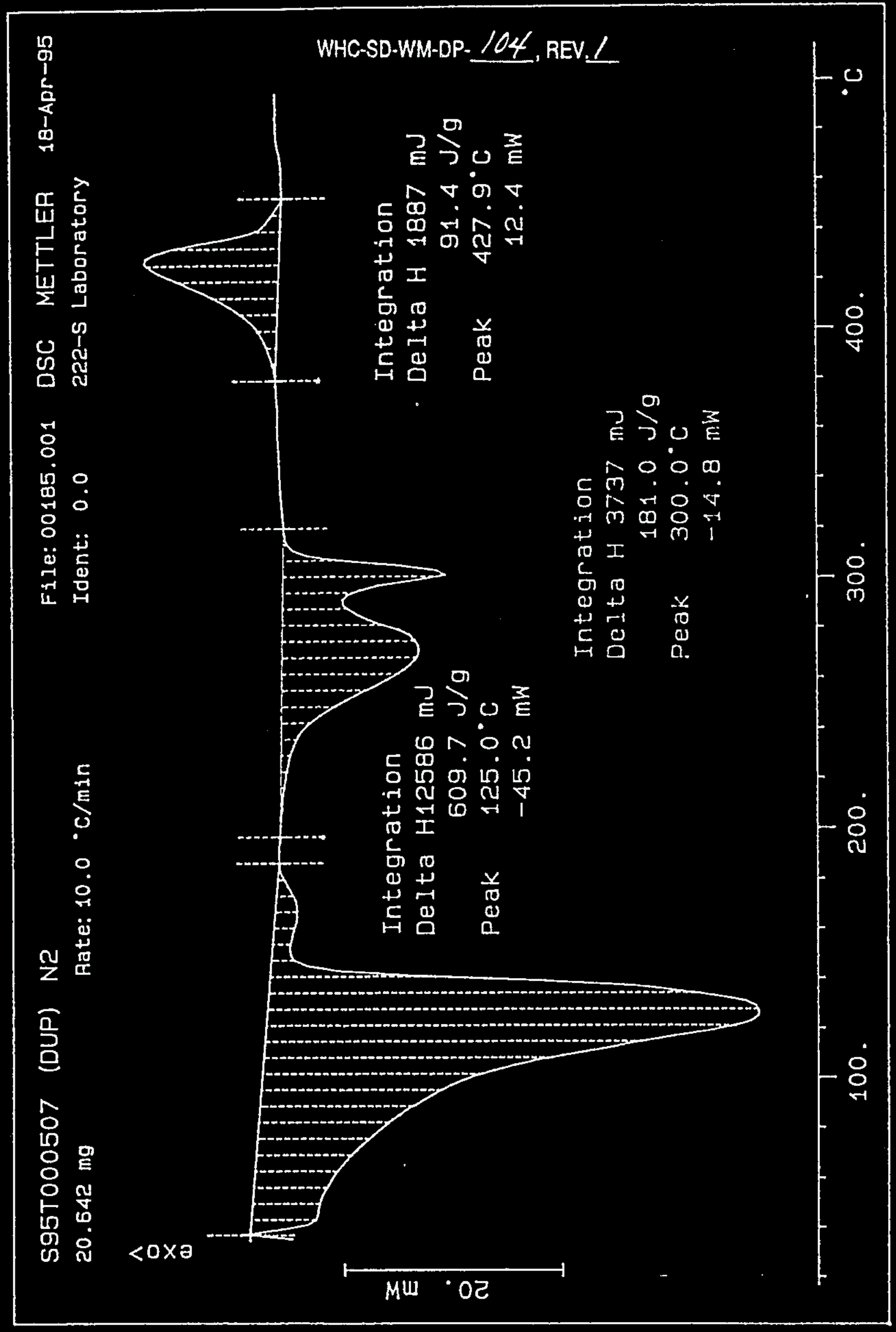




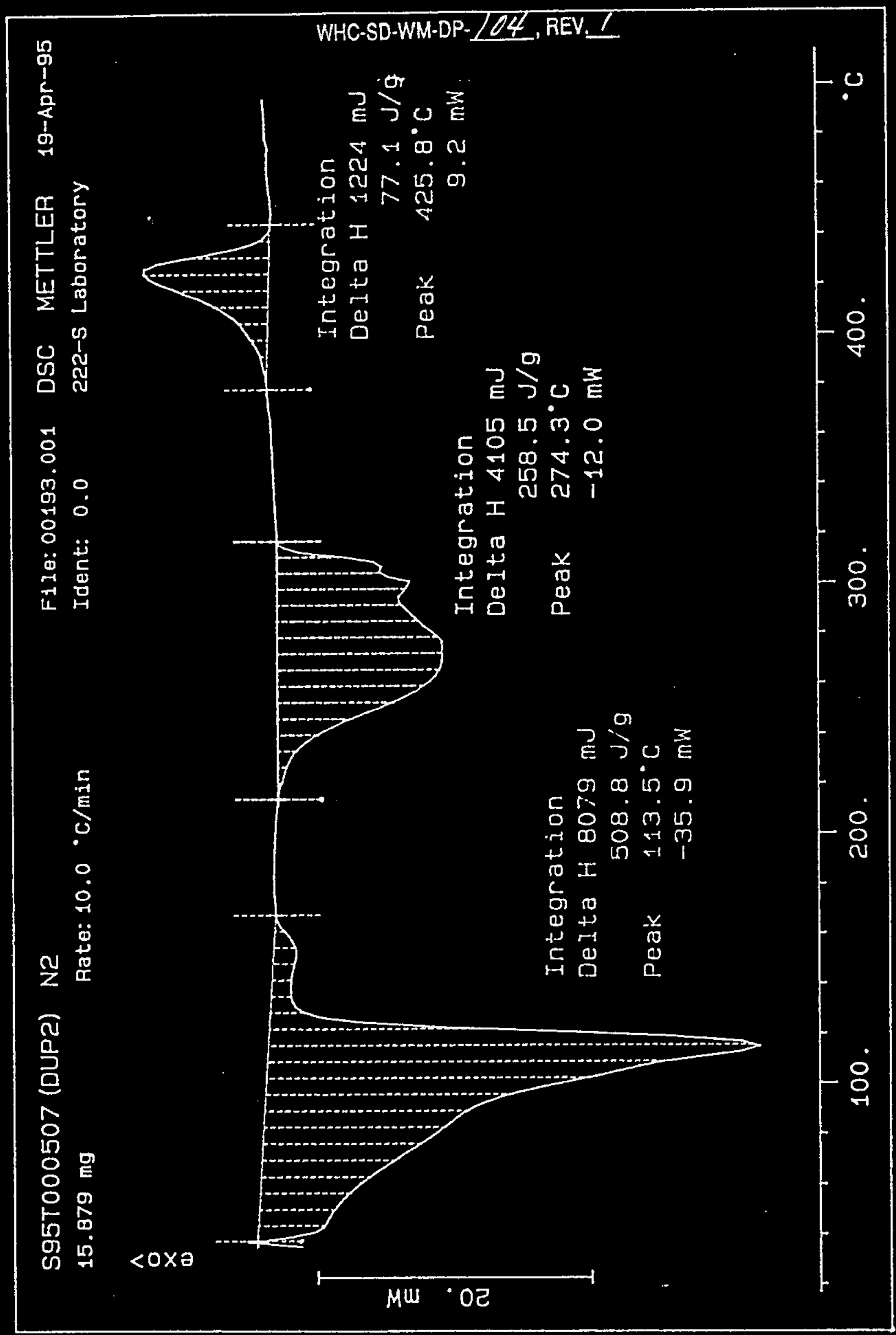




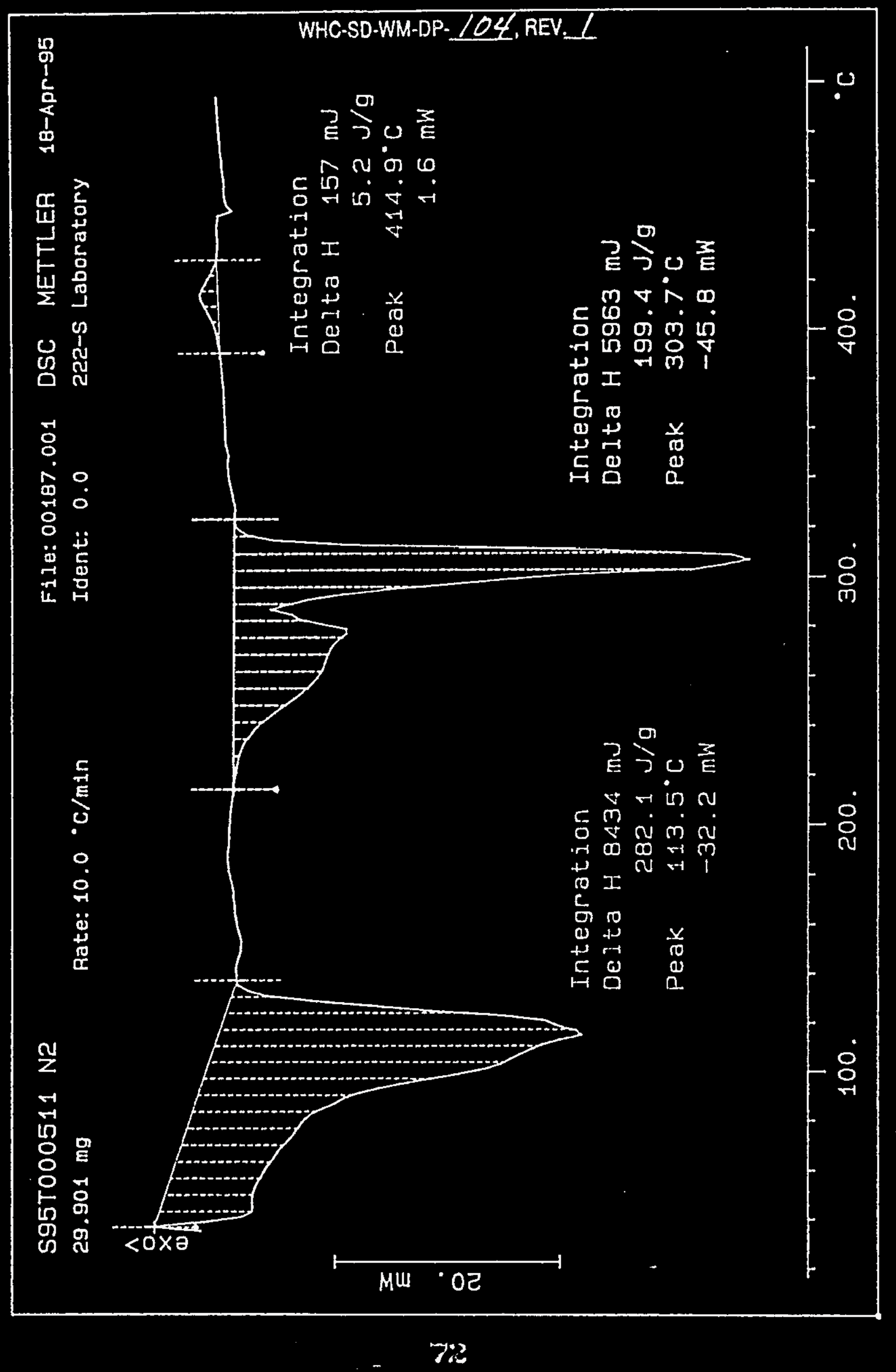




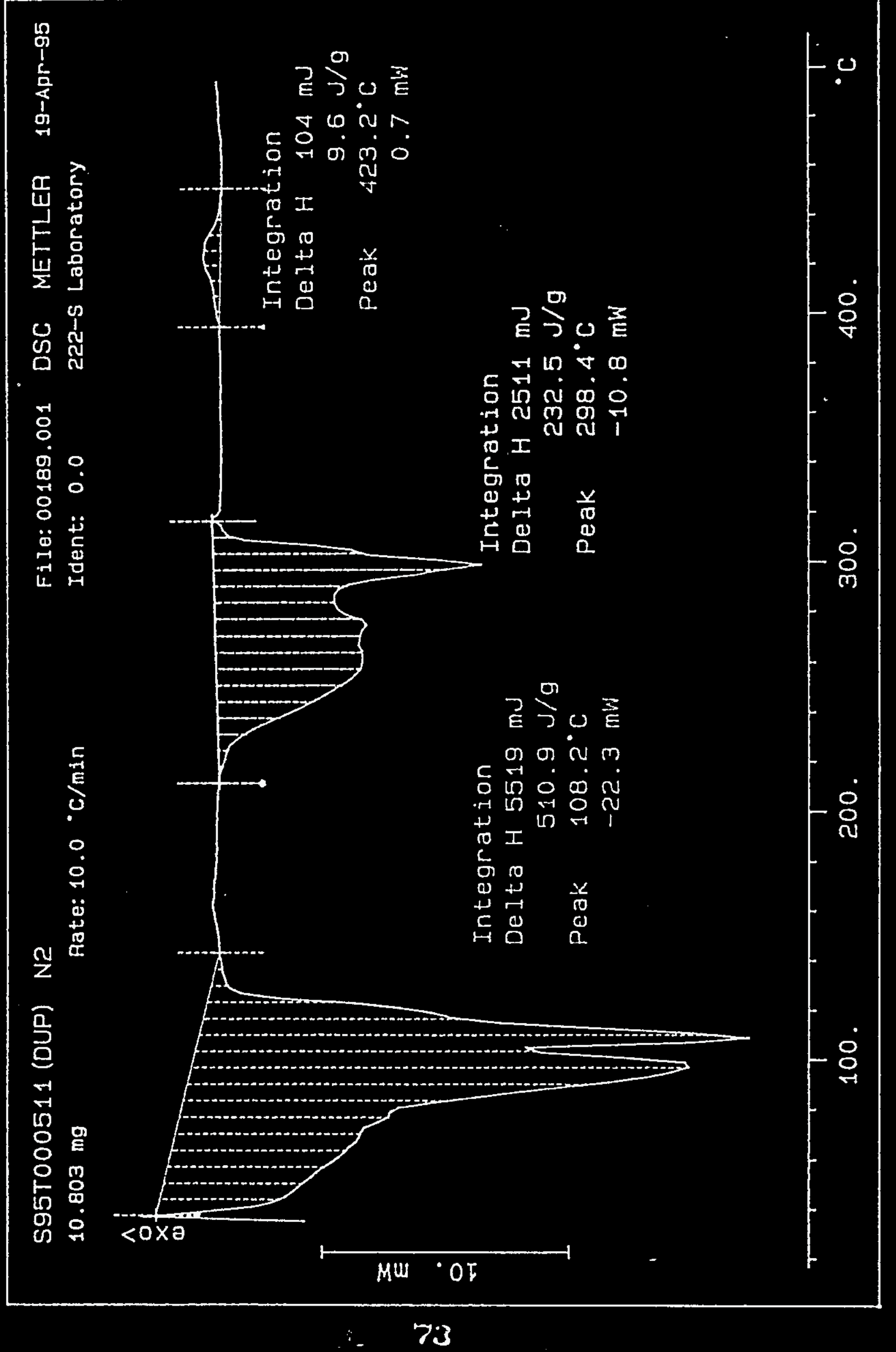


WLL SD-WM-DP. 104 REV. 1

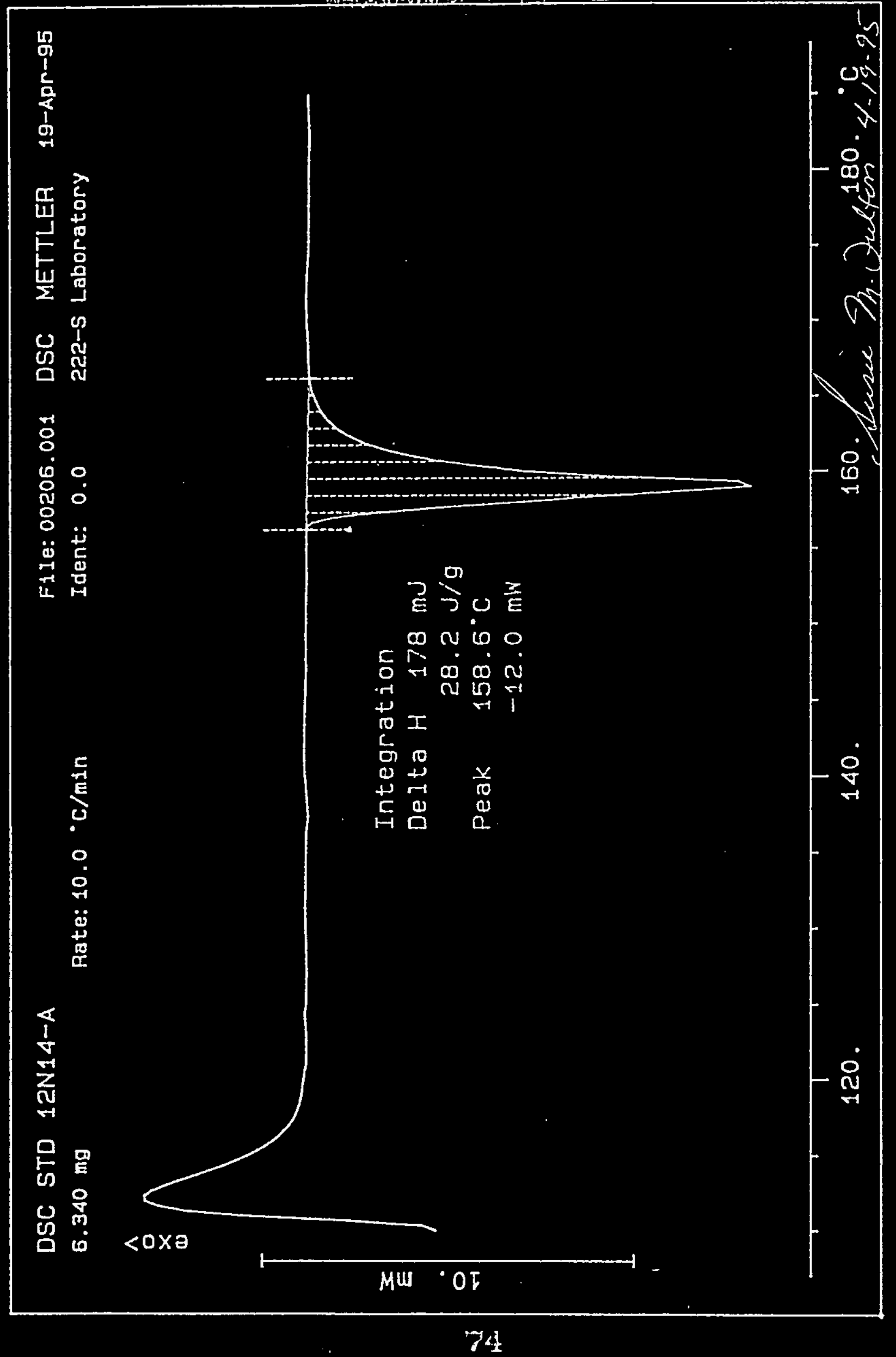


WHC.SD-WM-DP-104, REV.L

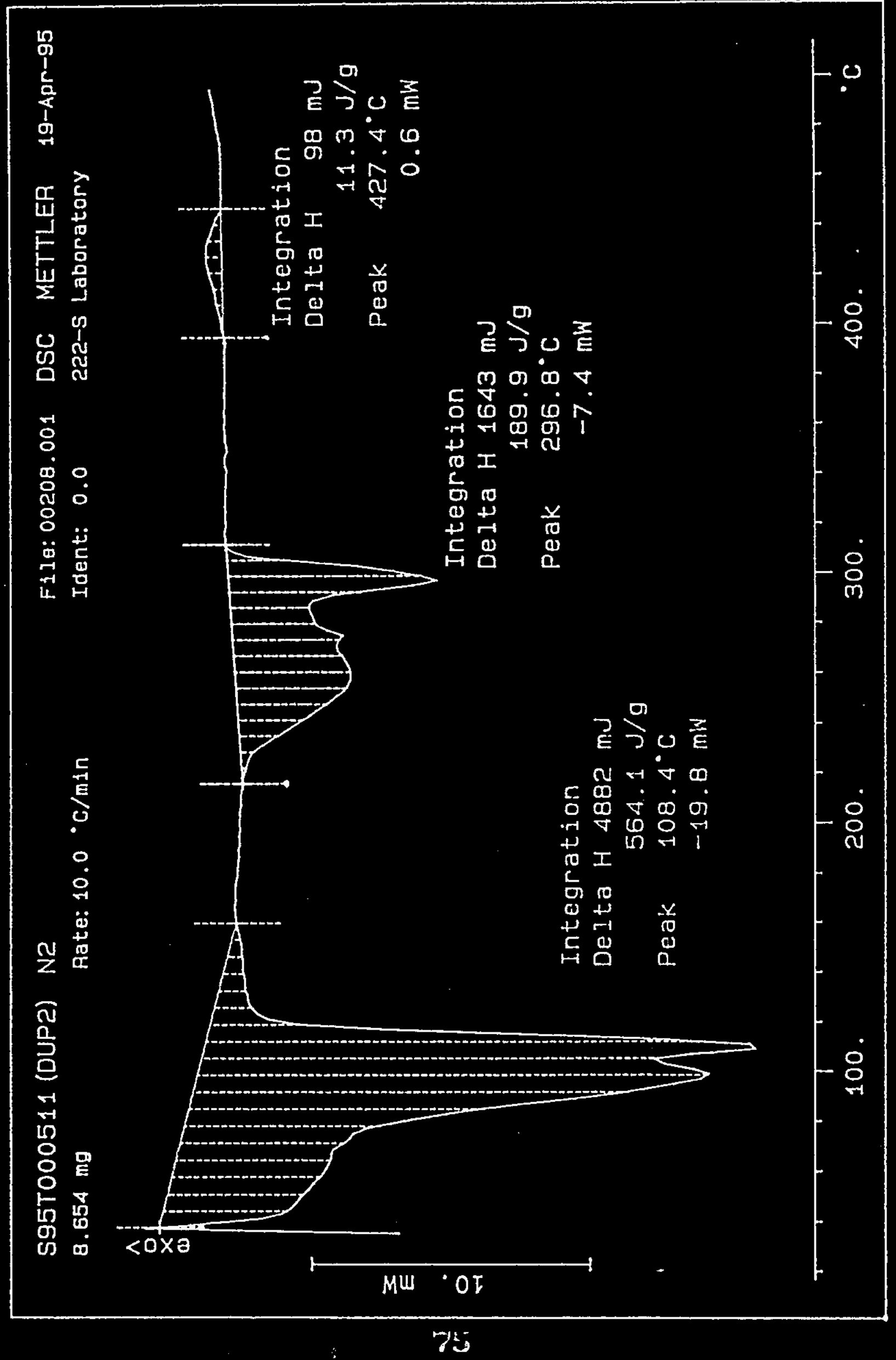




\section{LABCORE Data Entry Template for Worklist\# 945}

Analyst: $\quad K R M \quad$ Instrument: TGA01 — Book \# 42080

Method: LA-560-112 Rev/Mod A-2

Worklist Comment: Please run BY-103 TGA under N2. bdv

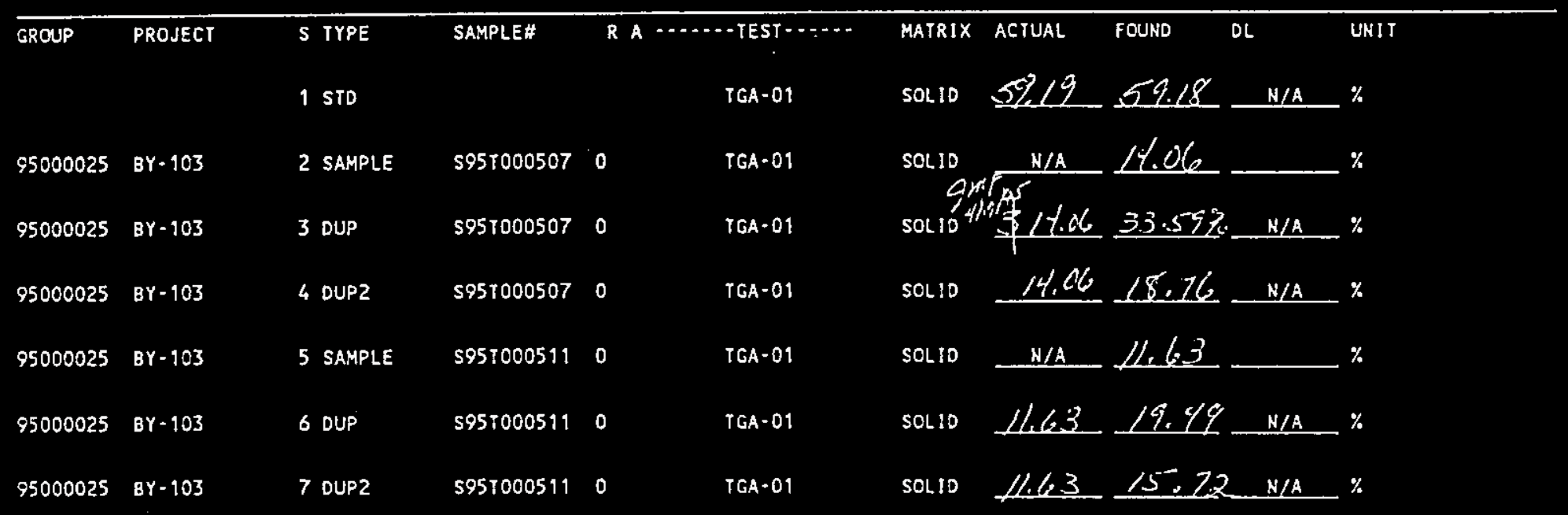

\section{Final page for worklist \# $945 \quad 4-19.95<\mathrm{AD}$}

\section{Su puttackedshectofor etiguxl}

Analyst Signature Datg signatece

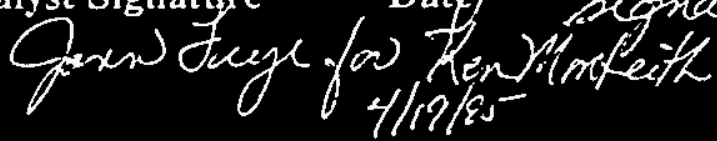

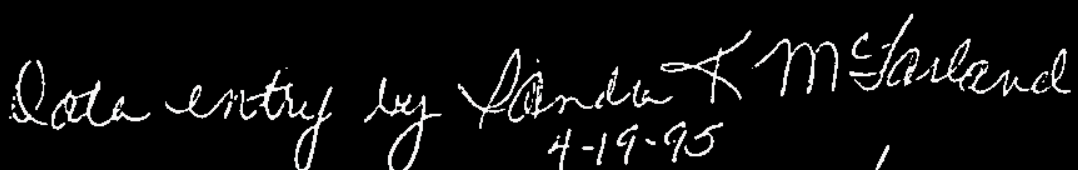
Verefied

Data Entry Comments:

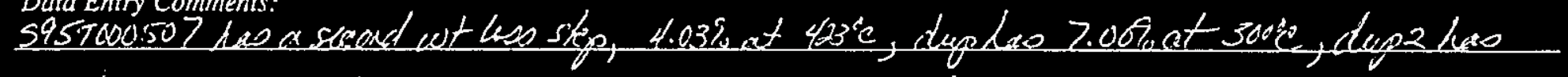

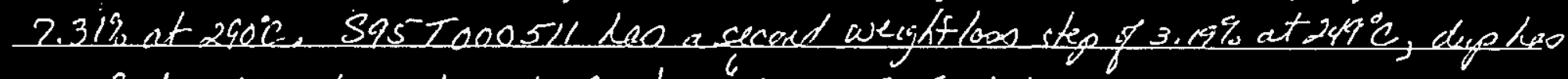

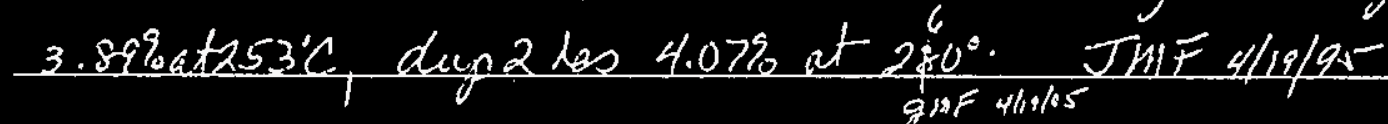

Units shown for $Q C$ (SPK \& STD) may not reflect the actual units. DL = Detection Limit, $S=$ Worklist Slot Number, $R=$ Replicate Number, $A=$ Aliguot Code. 


\section{LABCORE Data Entry Template for Worklist\# 945}

Analyst: $\quad K h M$ Instrument: TGA01

Method: LA-560-112 Rev/Mod
Book \#42N8A

WHC-SD-WM-DP. 104, REV. 1

Worklist Comment: Please run BY-103 TGA under N2. bdv

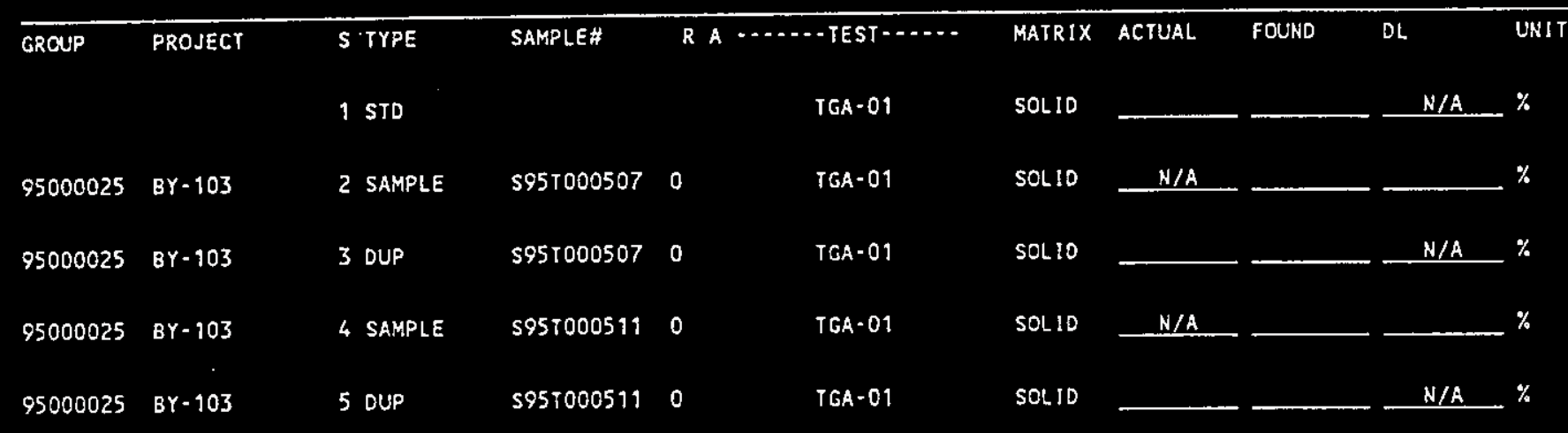

\section{Final page for worklist \# 945}

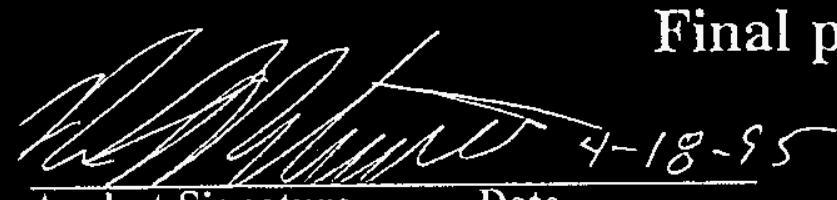

Analyst Signature
Date

Analyst Signature Date

Data Entry Comments:

j957000507 finar Brown

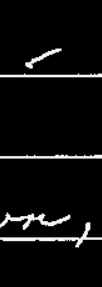

9957000511 very lighe bevere,

Units shown for $Q C$ (SPK \& STD) may not reflect the actual units. $D L=$ Detection Limit, $S=$ Worklist Slot Number,

$R=$ Replicate Number, $A=$ Aliquot Code. 


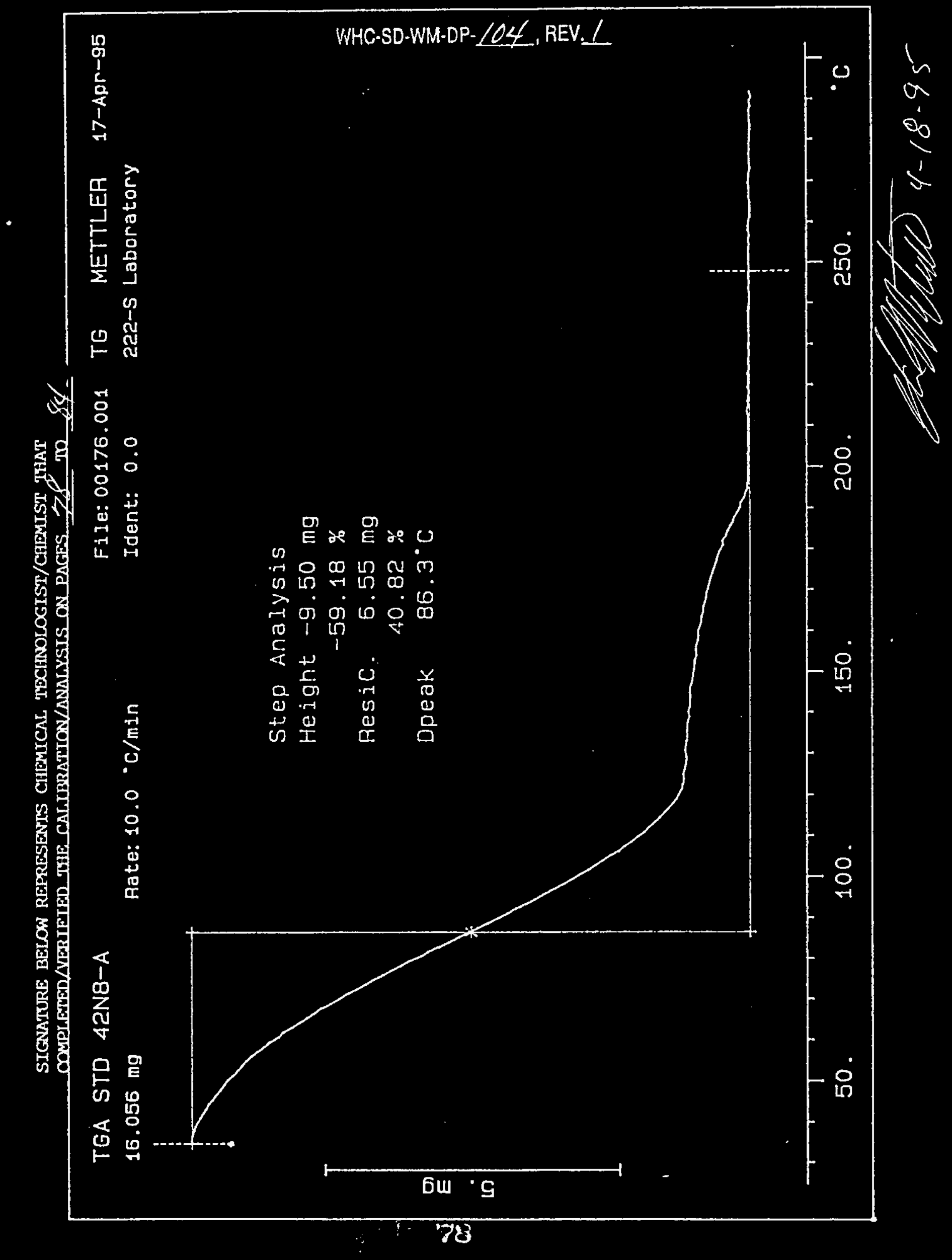




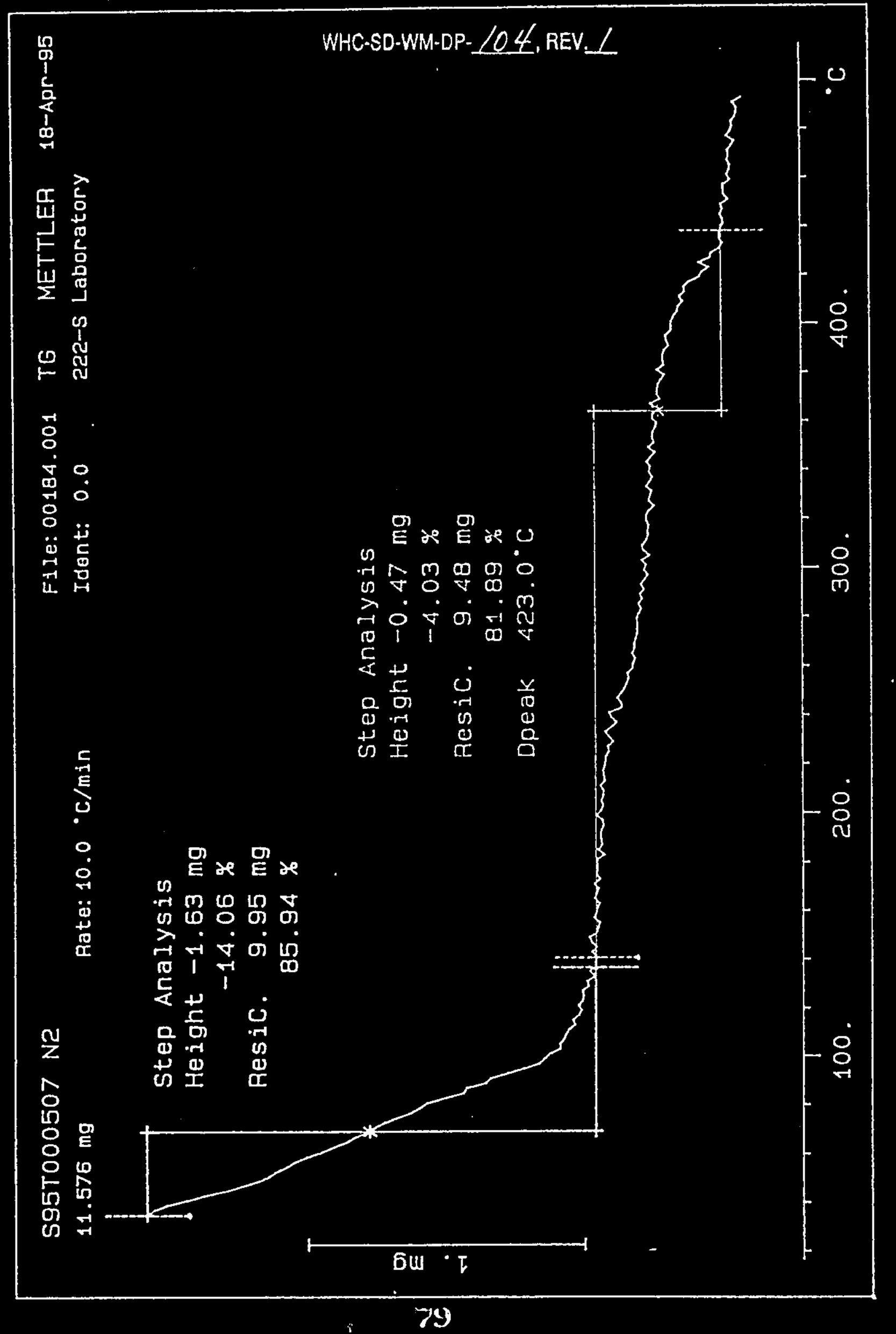




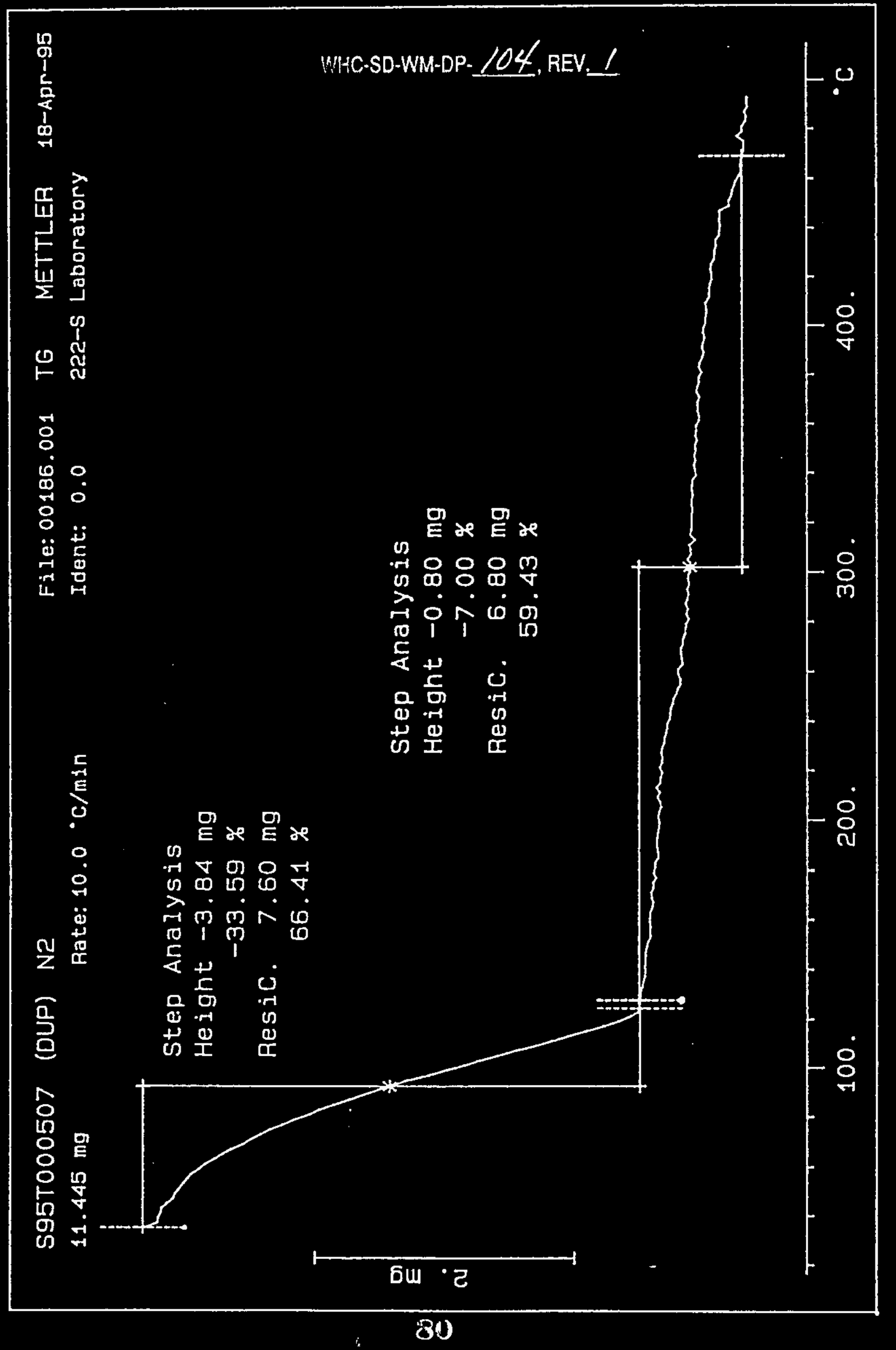




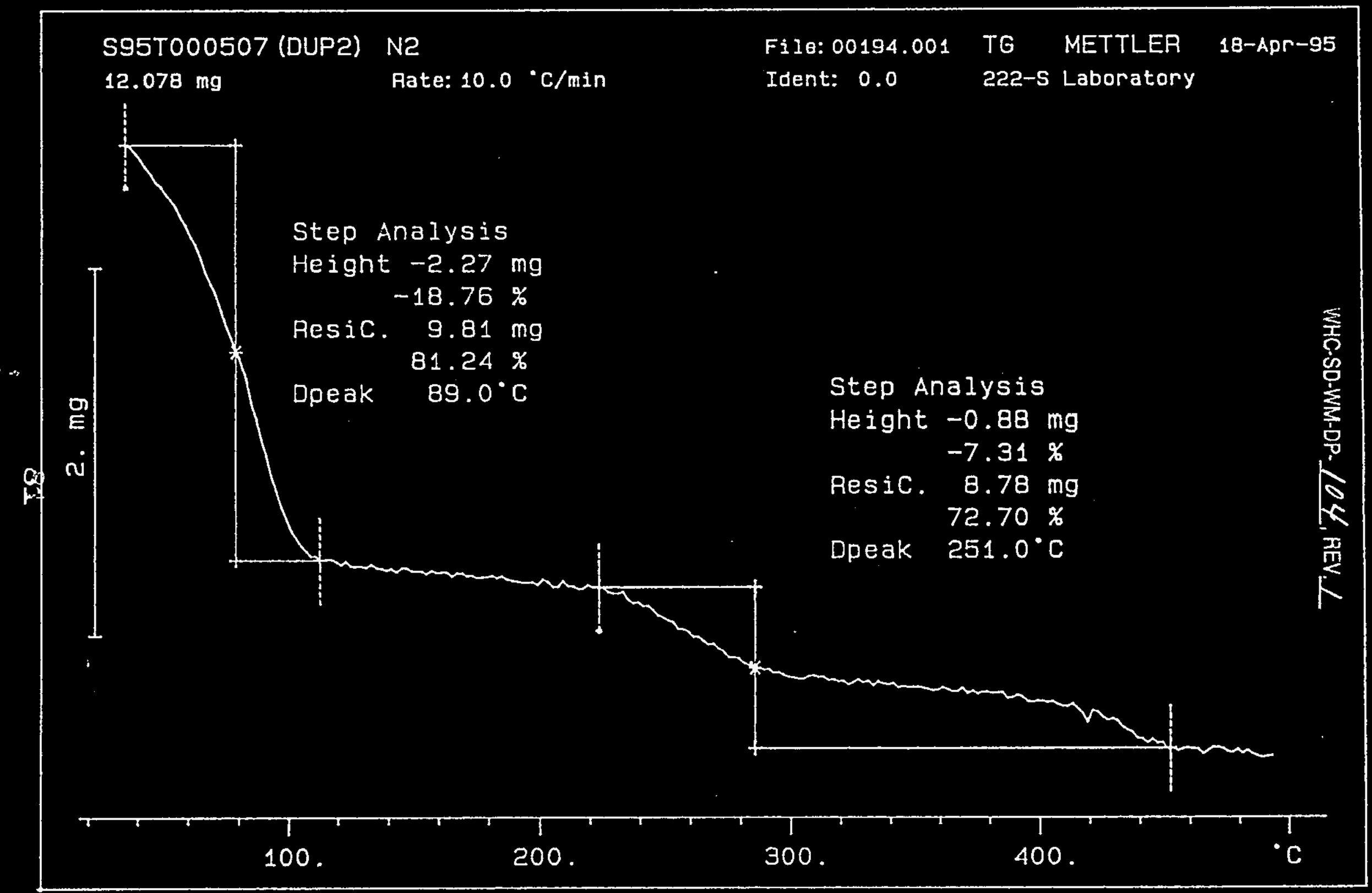




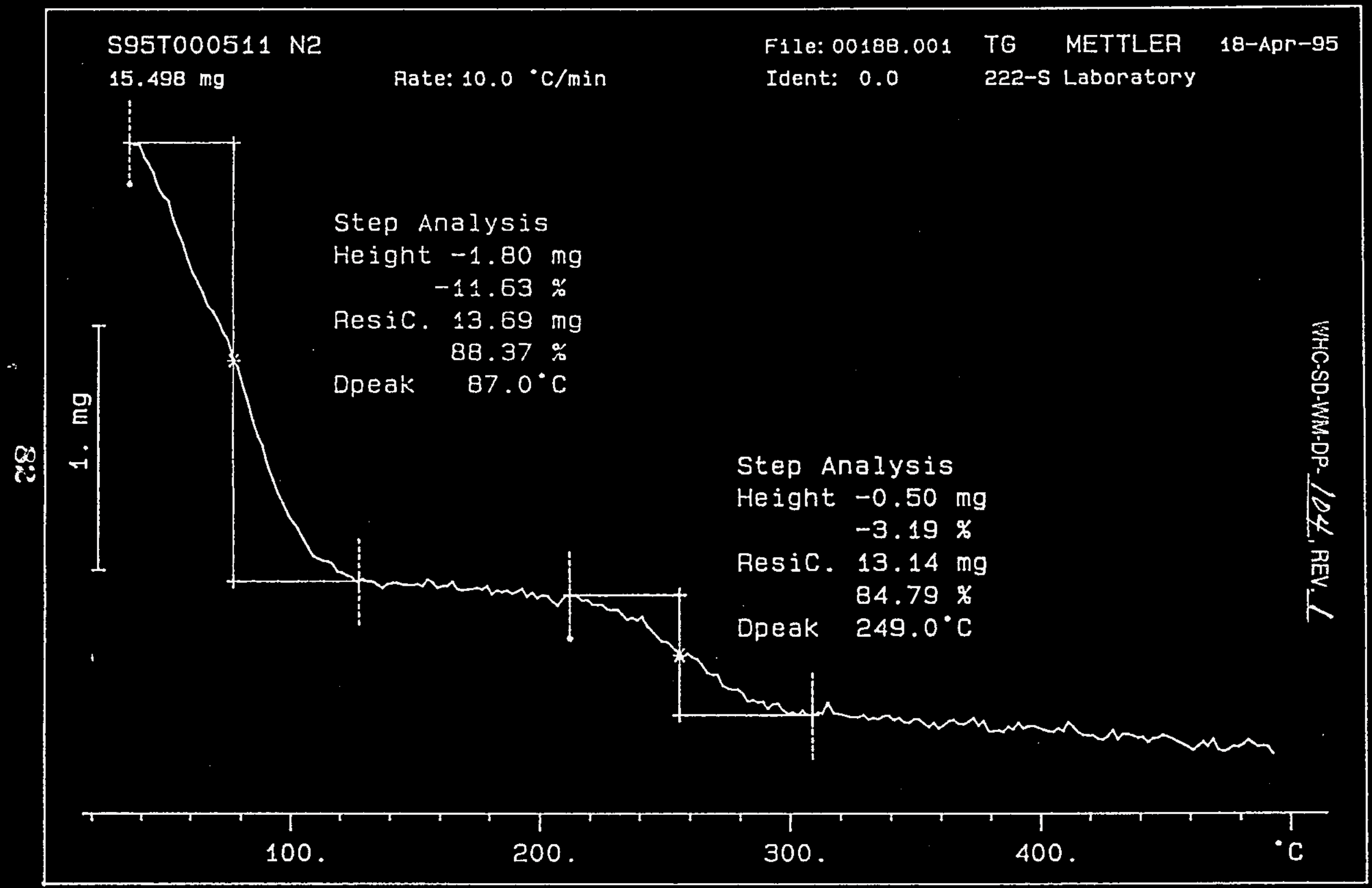




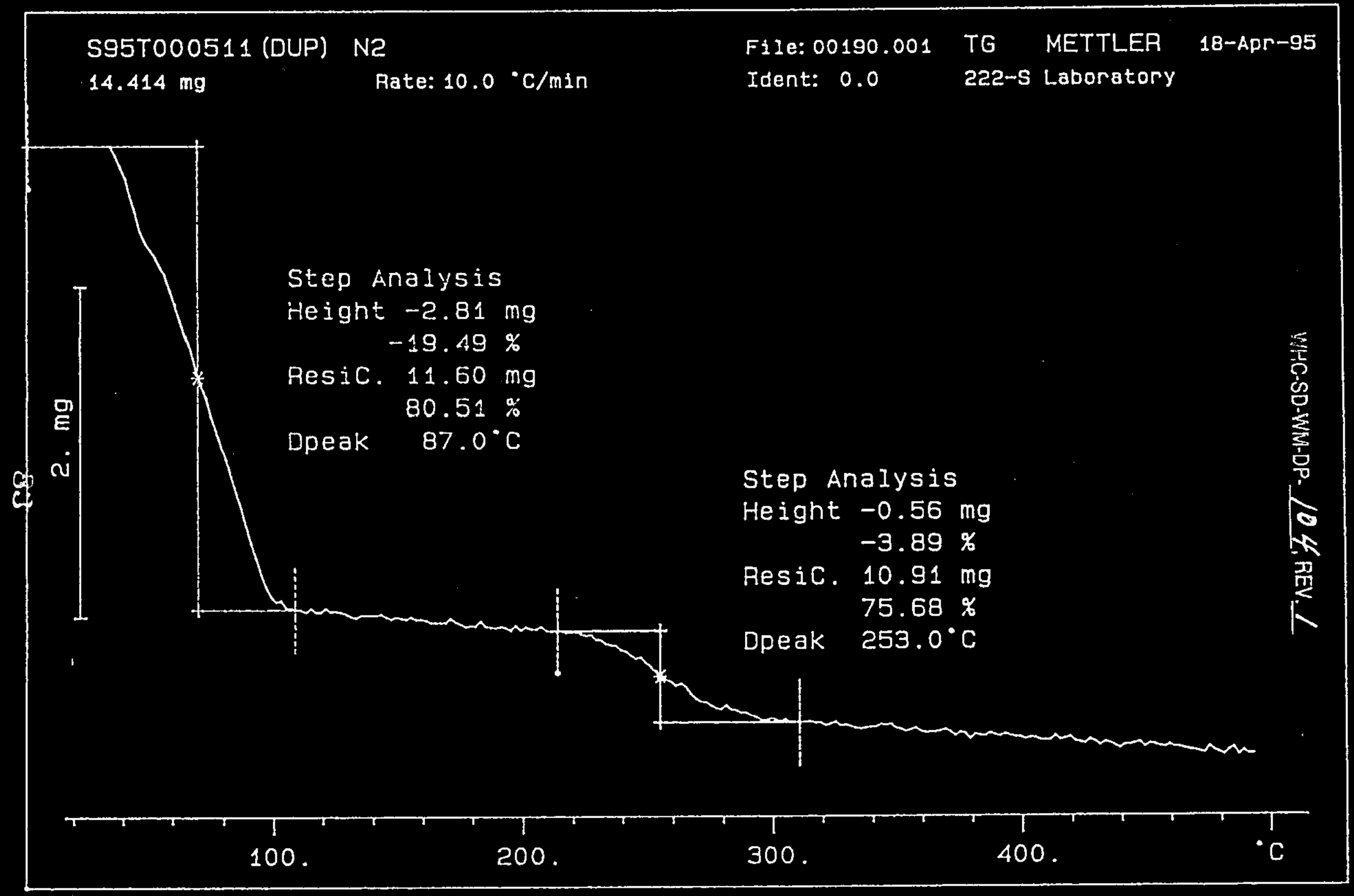




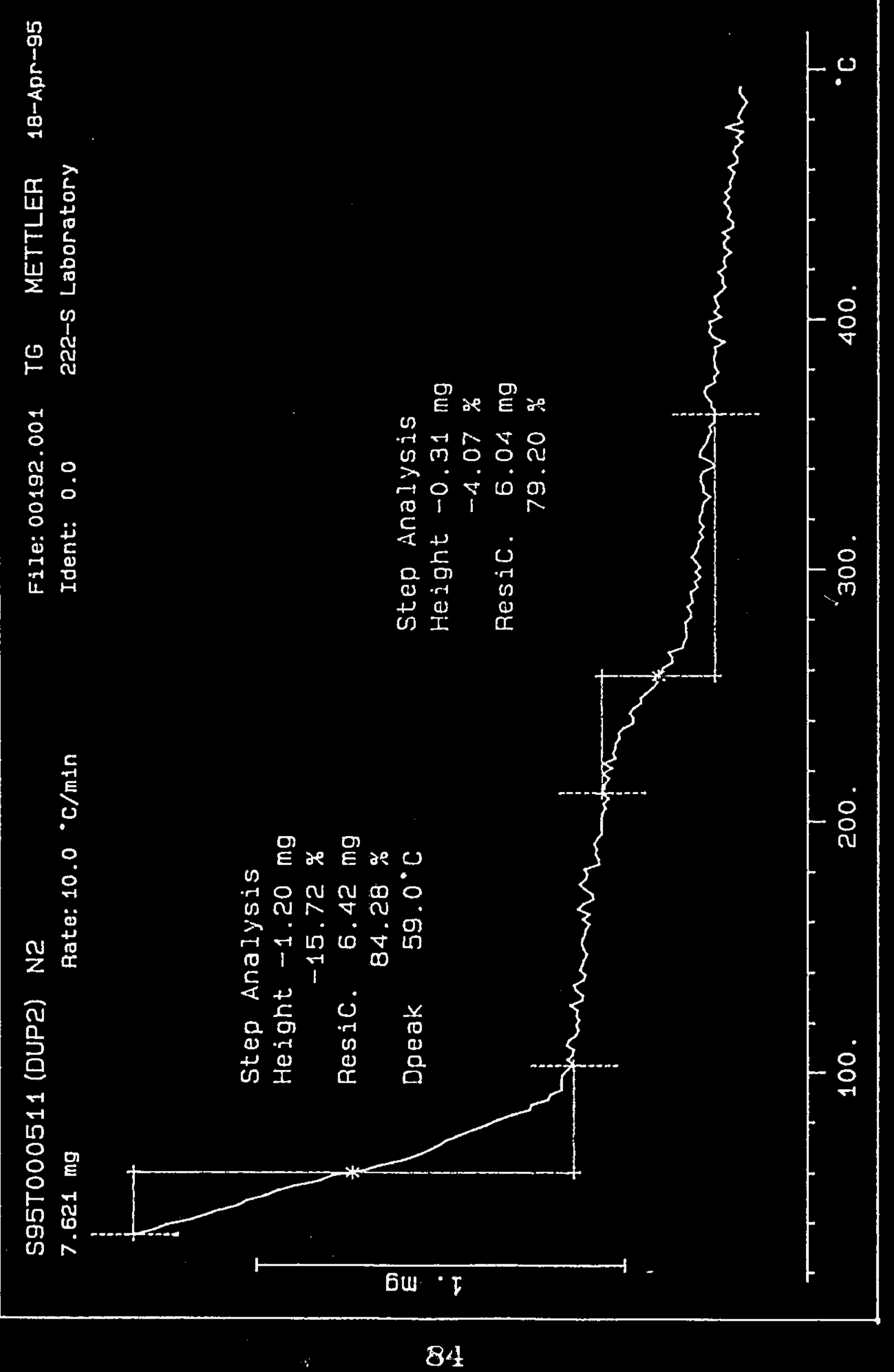

